\title{
PLANETS AROUND LOW-MASS STARS (PALMS). V. AGE-DATING LOW-MASS COMPANIONS TO MEMBERS AND INTERLOPERS OF YOUNG MOVING GROUPS*†‡§
}

\author{
Brendan P. Bowler ${ }^{1,19,21,22,23}$, Evgenya L. Shkolnik ${ }^{2,24}$, Michael C. Liu ${ }^{3,23}$, Joshua E. Schlieder ${ }^{4}$, Andrew W. ManN ${ }^{5}$, \\ Trent J. Dupuy ${ }^{5}$, Sasha Hinkley ${ }^{6}$, Justin R. Crepp ${ }^{7}$, John Asher Johnson ${ }^{8}$, Andrew W. Howard ${ }^{3}$, Laura FlagG ${ }^{2,9}$, \\ Alycia J. Weinberger ${ }^{10}$, Kimberly M. Aller ${ }^{3}$, Katelyn N. Allers ${ }^{11}$, William M. J. Best ${ }^{3}$, Michael C. Kotson ${ }^{3}$, \\ Benjamin T. Montet ${ }^{1,8,20}$, Gregory J. Herczeg ${ }^{12}$, Christoph Baranec ${ }^{3}$, Reed Riddle ${ }^{1}$, Nicholas M. Law ${ }^{13}$, \\ Eric L. Nielsen $^{14,15}$, Zahed Wahhaj ${ }^{16}$, Beth A. Biller ${ }^{17}$, and Thomas L. Hayward ${ }^{18}$ \\ ${ }^{1}$ California Institute of Technology, 1200 E. California Blvd., Pasadena, CA 91125, USA; bpbowler@caltech.edu \\ ${ }^{2}$ Lowell Observatory, 1400 W. Mars Hill Road, Flagstaff, AZ 86001, USA \\ ${ }^{3}$ Institute for Astronomy, University of Hawai 'i at Mānoa; 2680 Woodlawn Drive, Honolulu, HI 96822, USA \\ ${ }^{4}$ NASA Postdoctoral Program Fellow, NASA Ames Research Center, MS-245-3, Moffett Field, CA 94035, USA \\ ${ }_{6}^{5}$ Department of Astronomy, University of Texas at Austin, TX, USA \\ ${ }^{6}$ Physics and Astronomy, University of Exeter, EX4 4QL Exeter, UK \\ ${ }^{7}$ Department of Physics, University of Notre Dame, 225 Nieuwland Science Hall, Notre Dame, IN 46556, USA \\ ${ }^{8}$ Harvard-Smithsonian Center for Astrophysics, 60 Garden Street, Cambridge, MA 02138 USA \\ ${ }^{9}$ Department of Physics and Astronomy, Northern Arizona University, P.O. Box 6010, Flagstaff, AZ 86011, USA \\ ${ }^{10}$ Department of Terrestrial Magnetism, Carnegie Institution of Washington, 5241 Broad Branch Rd NW, Washington, DC 20015 USA \\ ${ }^{11}$ Department of Physics and Astronomy, Bucknell University, Lewisburg, PA 17837, USA \\ ${ }^{12}$ Kavli Institute for Astronomy and Astrophysics, Peking University; Yi He Yuan Lu 5, Hai Dian Qu; Beijing 100871, P. R. China \\ ${ }^{13}$ Department of Physics and Astronomy, University of North Carolina at Chapel Hill, Chapel Hill, NC 27599-3255, USA \\ ${ }^{14}$ Carl Sagan Center, SETI Institute, 189 Bernardo Avenue, Mountain View, CA 94043, USA \\ ${ }^{15}$ Kavli Institute for Particle Astrophysics and Cosmology, Stanford University, Stanford, CA 94305, USA \\ ${ }^{16}$ European Southern Observatory, Alonso de Cordova 3107, Vitacura, Santiago, Chile \\ ${ }^{17}$ Institute for Astronomy, University of Edinburgh, Blackford Hill View, Edinburgh EH9 3HJ, UK \\ ${ }^{18}$ Gemini Observatory, Southern Operations Center c/o AURA, Casilla 603, La Serena, Chile \\ Received 2015 February 24; accepted 2015 April 4; published 2015 June 9
}

\begin{abstract}
We present optical and near-infrared adaptive optics (AO) imaging and spectroscopy of 13 ultracool (>M6) companions to late-type stars (K7-M4.5), most of which have recently been identified as candidate members of nearby young moving groups (YMGs; 8-120 Myr) in the literature. Three of these are new companions identified in our AO imaging survey, and two others are confirmed to be comoving with their host stars for the first time. The inferred masses of the companions $\left(\sim 10-100 M_{\text {Jup }}\right)$ are highly sensitive to the ages of the primary stars; therefore we critically examine the kinematic and spectroscopic properties of each system to distinguish bona fide YMG members from old field interlopers. The new M7 substellar companion 2MASS J02155892-0929121 C (40-60 M Jup) shows clear spectroscopic signs of low gravity and, hence, youth. The primary, possibly a member of the $\sim 40 \mathrm{Myr}$ Tuc-Hor moving group, is visually resolved into three components, making it a young low-mass quadruple system in a compact ( $\lesssim 100 \mathrm{AU}$ ) configuration. In addition, Li I $\lambda 6708$ absorption in the intermediate-gravity M7.5 companion 2MASS J15594729+4403595 B provides unambiguous evidence that it is young ( $\lesssim 200 \mathrm{Myr})$ and resides below the hydrogen-burning limit. Three new close-separation $\left(<1^{\prime \prime}\right)$ companions (2MASS J06475229-2523304 B, PYC $\mathrm{J} 11519+0731 \mathrm{~B}$, and $\mathrm{GJ} 4378 \mathrm{Ab}$ ) orbit stars previously reported as candidate YMG members, but instead are likely old ( $\gtrsim 1 \mathrm{Gyr})$ tidally locked spectroscopic binaries without convincing kinematic associations with any known moving group. The high rate of false positives in the form of old active stars with YMG-like kinematics underscores the importance of radial velocity and parallax measurements to validate candidate young stars identified via proper motion and activity selection alone. Finally, we spectroscopically confirm the cool temperature and substellar nature of HD 23514 B, a recently discovered M8 benchmark brown dwarf orbiting the dustiest-known member of the Pleiades.
\end{abstract}

\footnotetext{
* Some of the data presented herein were obtained at the W. M. Keck Observatory, which is operated as a scientific partnership among the California Institute of Technology, the University of California, and the National Aeronautics and Space Administration. The Observatory was made possible by the generous financial support of the W. M. Keck Foundation.

$\dagger$ Based on observations obtained at the Southern Astrophysical Research (SOAR) telescope, which is a joint project of the Ministério da Ciência, Tecnologia, e Inovação (MCTI) da República Federativa do Brasil, the U.S. National Optical Astronomy Observatory (NOAO), the University of North Carolina at Chapel Hill (UNC), and Michigan State University (MSU).

$\$$ Based in part on data collected at Subaru Telescope, which is operated by the National Astronomical Observatory of Japan.

$\S$ Based on observations collected at the European Organization for Astronomical Research in the Southern Hemisphere, Chile (ESO Program 090.A-9010(A)).

19 Caltech Joint Center for Planetary Astronomy Fellow.

${ }^{20}$ NSF Graduate Research Fellow.
}

\footnotetext{
${ }^{21}$ Visiting Astronomer, Cerro Tololo Inter-American Observatory, National Optical Astronomy Observatory, which is operated by the Association of Universities for Research in Astronomy under contract with the National Science Foundation.

${ }^{22}$ Visiting Astronomer, Kitt Peak National Observatory, National Optical Astronomy Observatory, which is operated by the Association of Universities for Research in Astronomy (AURA) under cooperative agreement with the National Science Foundation.

${ }^{23}$ Visiting Astronomer at the Infrared Telescope Facility, which is operated by the University of Hawaii under Cooperative Agreement NNX-08AE38A with the National Aeronautics and Space Administration, Science Mission Directorate, Planetary Astronomy Program.

${ }^{24}$ Based on observations obtained at the Canada-France-Hawaii Telescope (CFHT), which is operated by the National Research Council of Canada, the Institut National des Sciences de l'Univers of the Centre National de la Recherche Scientifique of France, and the University of Hawaii.
} 
Key words: binaries: spectroscopic - brown dwarfs - stars: individual (2MASS J02155892, 0929121, 2MASS J15594729+4403595, HD 23514)

\section{INTRODUCTION}

With masses between $\approx 13-75 M_{\text {Jup }}$, brown dwarfs directly link the lowest-mass stars and young giant planets in mass, radius, and temperature. Nearly two thousand isolated brown dwarfs have been identified over the past 20 years, but their individual ages, metallicities, and masses are largely unknown. Age benchmarks-brown dwarfs orbiting wellcharacterized stars or members of coeval clusters-are rare but valuable tools to test low-temperature atmospheric and evolutionary models for mutual consistency and to empirically calibrate isolated field objects because their ages, distances, and compositions can be leveraged from the host star or cluster (e.g., Crepp et al. 2014; Deacon et al. 2014; Naud et al. 2014).

Over the past decade, dozens of nearby brown dwarfs with optical and near-infrared (NIR) spectroscopic signs of low surface gravity (youth) have been identified (e.g., Rebolo et al. 1995; Kirkpatrick 2008; Allers \& Liu 2013). Many of these objects have proper motions consistent with nearby $(<80 \mathrm{pc})$ young moving groups (YMGs) with ages of ₹10-120 Myr (e.g., Scholz et al. 2005; Looper et al. 2007; Delorme et al. 2012; Faherty et al. 2012; Gagné et al. 2014), but because they are intrinsically faint, most of these isolated brown dwarfs lack trigonometric distances and radial velocities (RVs) needed to unambiguously define their space motion. As a result, only a handful of young field brown dwarfs have been kinematically linked with high confidence to YMGs like TWA (Gizis et al. 2007; Weinberger et al. 2013), $\beta$ Pic (Liu et al. 2013b), and AB Dor (Faherty et al. 2012; Liu et al. 2013a). These low-gravity benchmarks are especially useful for empirical spectral classification sequences to map the effects of age on gravity-sensitive features like alkali absorption lines, $H$-band morphology, and $\mathrm{H}_{2} \mathrm{O}$ steam bands at $\approx 1.4$ and $\approx 1.9 \mu \mathrm{m}$ spanning a large range of temperatures $(500-2500 \mathrm{~K})$ and corresponding masses $\left(\approx 5-75 M_{\text {Jup }}\right)$. These empirically based spectral sequences at fixed ages also enable detailed analyses of secondary effects like photospheric clouds and nonequilibrium chemistry, which become increasingly important near the $\mathrm{L} / \mathrm{T}$ transition for young giant planets (Bowler et al. 2010; Barman et al. 2011; Marley et al. 2012; Skemer et al. 2014).

Another approach to find these elusive young substellar benchmarks is to search for faint companions to known nearby young stars. One advantage of this method is that precise parallaxes and RVs are more readily obtained for the primary stars, making kinematic association with YMGs relatively straightforward compared to faint isolated brown dwarfs in the field. Indeed, many substellar companions to YMG members have been discovered this way, primarily through space-based imaging (Lowrance et al. 1999; Webb et al. 1999; Lowrance et al. 2000) and adaptive optics (AO) imaging from the ground (Chauvin et al. 2005; Biller et al. 2010; Mugrauer et al. 2010; Wahhaj et al. 2011; Bowler et al. 2012a; Bowler et al. 2013; Delorme et al. 2013). However, old interloping stars with $U V W$ space motions similar to YMGs can complicate efforts to identify young substellar companions in moving groups. Distinguishing bona fide members from kinematic interlopers can be particularly challenging for M-type members of YMGs since both single mid- to late-M dwarfs and tidally locked $M+M$ binaries can have short rotation periods (less than a few days) and strong magnetic dynamos even at old ( $\gg 1$ Gyr) ages.

Here we present a suite of spectroscopic and AO imaging observations of ultracool companions to $\mathrm{M}$ dwarfs. The majority of these companions have been identified as part of the Planets Around Low-Mass Stars (PALMS) high-contrast imaging search for giant planets and brown dwarfs, three of which are new here (2MASS J06475229-2523304 B, PYC J11519+0731 B, and GJ 4378 Ab). Several additional systems were identified by the Astralux Lucky imaging survey (Janson et al. 2012), which obtained shallow diffraction-limited imaging of nearby, mostly active $M$ dwarfs in the optical. The sky positions, proper motions, spectral types, distances, and ages of our targets are listed in Table 1. The physical properties of the companions can be found in Table 2. Most of the primary stars have been previously reported in the literature as candidate or confirmed members of YMGs, making the companions excellent candidates for young benchmark brown dwarfs.

We critically examine these moving group membership claims on a case-by-case basis using new RVs of the primary stars together with recently published parallaxes, when available. We also use our new spectroscopy of the companions to independently constrain the ages of the systems via low-gravity spectroscopic features, which can be more prominent and last longer $(\lesssim 200 \mathrm{Myr})$ at late-M and L spectral types than at earlier types. Finally, we search for signs of orbital motion with multiepoch high-resolution AO imaging to identify systems that will yield dynamical masses in the near future.

\section{OBSERVATIONS}

\subsection{AO Imaging}

\subsubsection{Keck/NIRC2}

Our NIR imaging with NIRC2 at the $10 \mathrm{~m}$ Keck II telescope was carried out between 2012 and 2014 using Natural Guide Star AO (NGSAO; Wizinowich et al. 2000). We utilized the narrow NIRC2 camera mode, resulting in a plate scale of 9.952 \pm 0.002 mas pixel $^{-1}$ (Yelda et al. 2010) and a field of view (FOV) of $10.2^{\prime \prime} \times 10.2^{\prime \prime}$. The NIRC2 $J, H, K, K_{S}$, and $L^{\prime}$ filters are on the Maunakea Observatory (MKO) filter system (Simons \& Tokunaga 2002; Tokunaga \& Vacca 2005). The NIRC2 $Y$-band filter was installed in 2011 and has a central wavelength of $1.0180 \mu \mathrm{m}$ with a bandwidth of $0.0996 \mu \mathrm{m}$, differing slightly from other $Y$-band filters (Hillenbrand et al. 2002; see Liu et al. 2012 for additional details). In addition, we also include a single epoch of $K_{S}$-band NGSAO imaging of 1RXS J034231.8+121622 from 2007 in our analysis of the system (Section 3.4).

Raw images were cleaned of cosmic rays and bad pixels, dark-subtracted, flat-fielded, and north-aligned using the orientation of $+0.252^{\circ} \pm 0.009^{\circ}$ from Yelda et al. (2010). For our $L^{\prime}$ data, the science frames were combined after masking the target to create a flat field. For close companions $\left(\lesssim 2^{\prime \prime}\right)$, astrometry and relative photometry are measured by 
Table 1

Target Properties

\begin{tabular}{|c|c|c|c|c|c|c|c|c|c|c|c|c|c|}
\hline Name & $\alpha_{\mathrm{J} 2000}$ & $\delta_{\mathrm{J} 2000}$ & $\begin{array}{c}\mu_{\alpha} \cos \delta \\
\left(\mathrm{mas}_{\mathrm{yr}}^{-1}\right)\end{array}$ & 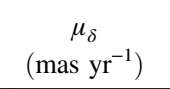 & $\begin{array}{c}\mu \\
\text { Ref. }\end{array}$ & $\begin{array}{c}\mathrm{SpT} \\
( \pm 0.5)\end{array}$ & $\begin{array}{l}\text { SpT } \\
\text { Ref. }\end{array}$ & $\begin{array}{c}\text { Distance } \\
(\mathrm{pc})\end{array}$ & $\begin{array}{c}\text { Dist. } \\
\text { Method }\end{array}$ & $\begin{array}{l}\text { Dist. } \\
\text { Ref. }\end{array}$ & $\mathrm{YMG}^{\mathrm{d}}$ & $\begin{array}{c}\text { Age } \\
(\mathrm{Myr})\end{array}$ & $\begin{array}{l}\text { Age } \\
\text { Ref. }\end{array}$ \\
\hline 2MASS J01225093-2439505 AB & 012250.93 & -243950.5 & $120.2 \pm 1.9$ & $-120.3 \pm 1.7$ & $\mathrm{Z13}$ & M4.0 & TW & $36 \pm 4$ & Phot & $\mathrm{B} 13$ & AB Dor? & $120 \pm 10$ & B13, M13 \\
\hline 2MASS J02155892-0929121 AabBC & 021558.92 & -092912.1 & $96.6 \pm 1.9$ & $-46.5 \pm 2.6$ & $\mathrm{Z13}$ & M3.5 & TW & $26 \pm 10$ & Phot & R06 & Tuc-Hor? & $\sim 30-40$ & M13, B15 \\
\hline 2MASS J02594789-0913109 AB & 025947.89 & -091310.9 & $296 \pm 8$ & $-527 \pm 8$ & $\mathrm{Z13}$ & M4.0 & R07 & $36 \pm 13$ & Phot & $\mathrm{J} 12$ & $\cdots$ & $\gtrsim 4000$ & TW \\
\hline 1RXS J034231.8+121622 AB & 034231.80 & +121622.5 & $196.8 \pm 2.2$ & $-16.3 \pm 3.8$ & Z13 & M4.0 & R06 & $23.9 \pm 1.1$ & $\pi$ & D14 & $\ldots$ & $60-300$ & Sh09 \\
\hline HD $23514 \mathrm{AB}$ & 034638.39 & +225511.2 & $20.5 \pm 0.4$ & $-42.6 \pm 0.5$ & $\mathrm{Z} 13$ & F5V & G01 & $136.2 \pm 1.2$ & Cluster & Me14 & Pleiades & $120 \pm 10$ & S98 \\
\hline G 160-54 AabBC & 041345.85 & -050904.9 & $182.0 \pm 8.0$ & $-112.5 \pm 8.0$ & $\mathrm{Z} 13$ & M4.5 & TW & $21 \pm 9$ & Phot & L11 & $\ldots$ & $\gtrsim 1000$ & TW \\
\hline 2MASS J05464932-0757427 AB & 054649.32 & -075742.7 & $127.9 \pm 2.2$ & $-53.8 \pm 2.1$ & Z13 & M3.0 & R07 & 45 & Phot & R07 & $\ldots$ & $\gtrsim 1000$ & TW \\
\hline 2MASS J06475229-2523304 AB & 064752.29 & -252330.4 & $22.7 \pm 0.8$ & $-72.0 \pm 1.1$ & Z13 & K7.0 & TW & $240 \pm 40$ & Phot & TW & $\cdots$ & $\ldots$ & $\cdots$ \\
\hline 2MASS J08540240-3051366 AB & 085402.40 & -305136.6 & $-281.6 \pm 5.7$ & $-8.9 \pm 5.7$ & R10 & M4.0 & R06 & $10 \pm 4$ & Phot & R06 & $\cdots$ & $\lesssim 200 ?$ & $\mathrm{C} 10$ \\
\hline 2MASS J11240434+3808108 AB & 112404.34 & +380810.8 & $121.7 \pm 2.6$ & $-12.4 \pm 2.6$ & Z13 & M4.5 & R07 & $20.3 \pm 1.3$ & Phot & B15 & UMa? & $500 \pm 100$ & Sh12 \\
\hline PYC J11519+0731 AabB & 115156.81 & +073126.2 & $-142 \pm 19$ & $-89 \pm 18$ & R08 & M2.0 & TW & $37 \pm 6$ & Phot & $\mathrm{TW}$ & $\cdots$ & $>10$ & TW \\
\hline G $180-11 \mathrm{AB}$ & 155531.78 & +351202.8 & $-233 \pm 8.0$ & $150.0 \pm 8.0$ & $\mathrm{Z13}$ & M4.0 & R06 & $28 \pm 3$ & $\pi$ & D14 & $\cdots$ & $\lesssim 200$ & TW \\
\hline 2MASS J15594729+4403595 AB & 155947.29 & +440359.5 & $-70.7 \pm 0.9$ & $-8.9 \pm 0.6$ & $\mathrm{Z} 13$ & M1.5 & TW & $27 \pm 2$ & Phot & $\mathrm{B} 15$ & $\cdots$ & $50-200$ & B15 \\
\hline GJ 4378 Aa12b & 235720.56 & -125848.7 & $208.3 \pm 8.0$ & $25.2 \pm 8.0$ & Z13 & M4.5 & TW & $14 \pm 2$ & Phot & $\mathrm{TW}$ & $\cdots$ & $\gtrsim 4000$ & $\mathrm{TW}$ \\
\hline
\end{tabular}

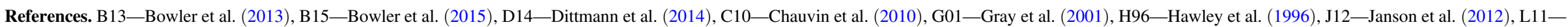

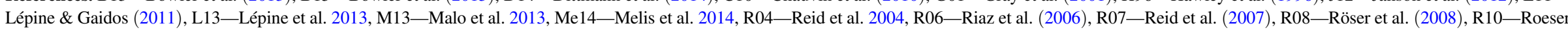
et al. (2010), S98-Stauffer et al. (1998), Sh09—Shkolnik et al. (2009), Sh12—Shkolnik et al. (2012), TW—This work, Z05—Zacharias et al. (2005), Z13-UCAC4 (Zacharias et al. 2013). 
Table 2

Companion Properties

\begin{tabular}{|c|c|c|c|c|c|c|c|}
\hline Name & $\begin{array}{c}\text { Ang. Sep } \\
\left({ }^{\prime \prime}\right)\end{array}$ & $\begin{array}{c}\text { Proj. Sep } \\
(\mathrm{AU})\end{array}$ & $\mathrm{SpT}$ & YMG? & $\begin{array}{c}\text { Age } \\
(\mathrm{Myr})\end{array}$ & $\begin{array}{l}\text { Mass } \\
\left(M_{\text {Jup }}\right)\end{array}$ & $\begin{array}{l}\text { Discovery } \\
\text { Reference }\end{array}$ \\
\hline 2MASS J01225093-2439505 B & 1.5 & $52 \pm 6$ & $\mathrm{~L} 5 \pm 1$ & AB Dor? & $120 \pm 10$ & $13-25$ & B13 \\
\hline 2MASS J02155892-0929121 C & 3.5 & $90 \pm 10$ & $\mathrm{M} 7 \pm 1$ & Tuc-Hor? & $30-40$ & $42 \pm 15$ & B10, TW \\
\hline 1RXS J034231.8+121622 B & 0.8 & $19.1 \pm 0.9$ & $\mathrm{~L} 0 \pm 1$ & $\cdots$ & $60-300$ & $35 \pm 8$ & B15 \\
\hline HD 23514 B & 2.6 & $360 \pm 3$ & $\mathrm{M} 8 \pm 1$ & Pleiades & $120 \pm 10$ & $60 \pm 10$ & $\mathrm{R} 12$ \\
\hline G $160-54 \mathrm{C}$ & 3.3 & $70 \pm 30$ & $\mathrm{M} 7.0 \pm 0.5$ & $\cdots$ & $\gtrsim 1000$ & $85 \pm 16$ & B15 \\
\hline 2MASS J08540240-3051366 B & 1.7 & $17 \pm 7$ & $\mathrm{M} 5.0 \pm 0.5$ & $\cdots$ & $\lesssim 200 ?$ & $\sim 40 \pm 20$ & $\mathrm{C} 10$ \\
\hline 2MASS J11240434+3808108 B & 8.3 & $170 \pm 11$ & $\mathrm{M} 9.5 \pm 0.5$ & UMa? & $500 \pm 100$ & $81 \pm 5$ & $\mathrm{C} 03$ \\
\hline PYC J11519+0731 B & 0.5 & $18 \pm 3$ & $\mathrm{M} 8 \pm 1$ & $\cdots$ & $>10$ & $15-90$ & TW \\
\hline G $180-11$ B & 1.6 & $45 \pm 5$ & $\mathrm{M} 6.0 \pm 0.5$ & $\cdots$ & $\lesssim 200$ & $90-150$ & M01, D07 \\
\hline 2MASS J15594729+4403595 B & 5.6 & $150 \pm 11$ & $\mathrm{M} 7.5 \pm 0.5$ & $\cdots$ & $50-200$ & $43 \pm 9$ & $\mathrm{~J} 12, \mathrm{~B} 15$ \\
\hline GJ $4378 \mathrm{Ab}$ & 0.5 & $7 \pm 1$ & $\mathrm{M} 8 \pm 1$ & $\cdots$ & $\gtrsim 4000$ & $90 \pm 3$ & TW \\
\hline
\end{tabular}

References. B10-Bergfors et al. (2010), B13-Bowler et al. (2013), B15-Bowler et al. (2015), C03-Close et al. (2003), C10—Chauvin et al. (2010), D07Daemgen et al. (2007), J12—Janson et al. (2012), M01—McCarthy et al. (2001), R12—Rodriguez et al. (2012), TW—This work.

${ }^{a}$ Estimated spectral type based on colors.

fitting three bivariate Gaussians to each binary component as described in Liu et al. (2008). Aperture photometry is used for companions widely separated from their host stars. The standard deviations from multiple measurements are adopted for our separation and position angle (P.A.) uncertainties.

For PYC J11519+0731 we also obtained deep imaging with the 600 mas diameter coronagraph in angular differential imaging mode (Liu 2004; Marois et al. 2006) in $H$ band on 2012 May 22 UT. We acquired 20 frames each with an integration time of $30 \mathrm{~s}$ spanning a total sky rotation of $11^{\circ}$. Following image registration based on the star's position behind the partly opaque mask and corrections for optical distortions (B. Cameron, 2007, private communication), we performed point-spread function (PSF) subtraction with the Locally Optimized Combination of Images algorithm (LOCI; Lafrenière et al. 2007) as described in Bowler et al. (2015). However, because of the small sky rotation, we also used a reference PSF library containing over 2000 NIRC2 coronagraphic images from our PALMS program. At each geometric subsection the LOCI coefficients were computed from 100 library images, which were chosen by minimizing the rms after scaling and subtracting every PSF reference image within that particular section. For our final reduction we masked the region surrounding PYC J11519+0731 B to avoid self-subtraction when computing image coefficients. Coronagraph throughput measurements from Bowler et al. (2015) were used to produce a $7 \sigma$ contrast curve. Results are described in Section 3.11.

\subsubsection{Subaru/Infrared Camera and Spectrograph}

We imaged 2MASS J02155892-0929121, 2MASS J06475229-2523304, and GJ 4378 A with the Infrared Camera and Spectrograph (IRCS; Tokunaga 1998; Kobayashi 2000) coupled with the AO188 NGSAO system (Hayano et al. 2010) at the $8.2 \mathrm{~m}$ Subaru Telescope on UT 2012 October 12 and 13. The weather was clear throughout both nights, but the seeing was highly variable $\left(1-2^{\prime \prime}\right)$. The A-B-C components of 2MASS J02155892-0929121 were easily identified, but the (then unknown) fourth member, $\mathrm{Ab}$, was not resolved in these data. We obtained MKO $K$-band images of 2MASS J02155892-0929121 ABC and MKO $J, H$, and $K$ images of 2MASS J06475229-2523304 AB and GJ 4378 Aab.

The raw frames were cleaned of bad pixels, flat-fielded, and dark-subtracted. Astrometry and relative photometry were measured for each image individually by fitting three bivariate Gaussians to each component (Liu et al. 2008). We adopt a plate scale and north orientation of $20.41 \pm 0.05$ mas pixel $^{-1}$ and $+89.03^{\circ} \pm 0.09^{\circ}$ measured in Bowler et al. (2013) using the 2.4" binary 1RXS J235133.3+312720 AB (Bowler et al. 2012a), which we observed with IRCS on UT 2012 October 12.

\subsubsection{Gemini/Near-Infrared Coronagraphic Imager}

On UT 2010 August 29 we targeted 1RXS J034231.8 +121622 with the Near-Infrared Coronagraphic Imager (NICI; Chun et al. 2008) on the $8.1 \mathrm{~m}$ Gemini South telescope in queue mode as part of the NICI Planet-Finding Campaign (Liu et al. 2010b; Program GS-2010B-Q-500, PI: M. Liu). NICI is a simultaneous dual-channel imager with a tapered, partly opaque Lyot coronagraph and two $1024 \times 1024$ $\left(18^{\prime \prime} \times 18^{\prime \prime} \mathrm{FOV}\right)$ infrared arrays. A short imaging sequence of eight $60 \mathrm{~s}$ frames was acquired for 1RXS J034231.8+121622 in $\mathrm{H}$ and $\mathrm{CH}_{4}$ long (4\% throughput centered at $1.652 \mu \mathrm{m}$ ) bandpasses. Ultimately, this target was not included in the final NICI Planet-Finding Campaign because the ADI sequence was aborted after a few frames. The $0.8^{\prime \prime}$ companion 1RXS J034231.8+121622 B originally found by Bowler et al. (2015) is easily detected in individual images without PSF subtraction.

PYC J11519+0731 was targeted in a similar fashion with NICI on UT 2013 April 19 as part of a separate survey to search for giant planets orbiting young $\mathrm{M}$ dwarfs (Program GS2013A-Q-54, PI: E. Nielsen). A total of 10 short (1.14 s per coadd $\times 10$ coadds) frames were obtained simultaneously in $H$ and $K_{S}$ bands, resulting in a total on-source integration time of $114 \mathrm{~s}$ per filter. PYC J11519+0731 B was easily resolved and remained unsaturated in these short images. 
We reduced the NICI images following the description in Wahhaj et al. (2011), which includes basic image reduction (bad pixel removal and flat-fielding) and distortion correction. Plate-scale and sky orientation values were used from calibration measurements by the Gemini staff closest in time before the observations were taken (Hayward et al. 2014). Astrometry and relative photometry incorporating the mask transmission measurement and uncertainty from Wahhaj et al. (2011) is listed in Table 3.

\subsubsection{Palomar $60 \mathrm{Inch} /$ Robo-AO}

2MASS J15594729+4403595，2MASS J11240434+3808108, and G 180-11 were imaged with the visible-light Robo-AO robotic laser guide star AO system (Baranec et al. 2013; Baranec et al. 2014) mounted on the automated Palomar 60 inch $(1.5 \mathrm{~m})$ telescope (Cenko et al. 2006) on the night of 2014 June 13 UT. Robo-AO utilizes a pulsed ultraviolet laser to generate a Rayleigh-scattered guide star for wavefront sensing, enabling efficient observations with AO correction in the optical down to $V \approx 16$ mag. The electron-multiplying CCD camera is continually read out during the observations, and a shift-and-add pipeline creates a final science image (Law et al. 2014). The nominal detector FOV is $44^{\prime \prime}$ with a plate scale of $0.0431^{\prime \prime}$ pixel $^{-1}$ (Baranec et al. 2014). The pipeline processing produces a final image that is resampled at half the original pixel scale $\left(0.0216^{\prime \prime}\right.$ pixel $\left.^{-1}\right)$.

All three targets were imaged in Sloan $r^{\prime}, i^{\prime}$, and $z^{\prime}$ filters (York et al. 2000) with exposure times of $60 \mathrm{~s}$ each. The late-M companions to 2MASS J15594729+4403595 and 2MASS $\mathrm{J} 11240434+3808108$ are clearly visible in the $i^{\prime}$ and $z^{\prime}$ images. $\mathrm{G} 180-11 \mathrm{~B}$ is detected in all three filters. The north orientation and plate scale are derived using the $z^{\prime}$ image of 2MASS J15594729+4403595 AB and astrometry of the pair from Bowler et al. (2015). Based on its photometric distance of $27 \mathrm{pc}$, this system has a projected separation of $\approx 150 \mathrm{AU}$. No orbital motion is expected, as the observations used for astrometric calibration were taken in 2012. We measure a plate scale of $0.0219^{\prime \prime} \pm 0.0002^{\prime \prime}$ pixel $^{-1}$ and sky orientation on the detector of $335.4^{\circ} \pm 0.4^{\circ}$. These are within $2 \sigma$ of the values derived using a distortion solution based on Hubble Space Telescope-calibrated images of M15 (A. Tokovinin, 2015, private communication). The FWHM of the primary stars in these observations are $\sim 0.28^{\prime \prime}, \sim 0.20^{\prime \prime}$, and $\sim 0.16^{\prime \prime}$ in $r^{\prime}, i^{\prime}$, and $z^{\prime}$, respectively, in $1.3^{\prime \prime}-1.8^{\prime \prime}$ seeing conditions. Flux ratios for the components are measured with aperture photometry and listed in Table 3.

\subsection{NIR Spectroscopy}

\subsubsection{Keck/OH-suppressing Infrared Imaging Spectrograph-NGSAO}

NIR spectroscopy of low-mass companions allows for spectral classification and provides an independent diagnostic of youth in the form of gravity-sensitive atomic and molecular features. We obtained moderate-resolution $(R \equiv \delta \lambda / \lambda=3800)$ NIR spectra of the close-in companions $\left(<3^{\prime \prime}\right) \quad$ PYC J11519+0731 B, G 180-11 B, HD $23514 \mathrm{~B}$, GJ $4378 \mathrm{Ab}$, 2MASS J06475229-2523304 B, and 2MASS J08540240 - 3051366 B with the OH-suppressing Infrared Imaging Spectrograph (OSIRIS; Larkin et al. 2006) at the $10 \mathrm{~m}$ Keck I telescope between 2012 June and 2014 December (Table 4). Our 2013 and 2014 observations utilized the newly installed, more efficient grating described in Mieda et al. (2014).
We used the 20 mas pixel $^{-1}$ plate scale $\left(0.32^{\prime \prime} \times 1.28^{\prime \prime} \mathrm{FOV}\right)$ for PYC J11519+0731 B， 2MASS J06475229-2523304 B , and 2MASS J08540240-3051366 B. The 50 mas pixel $^{-1}$ scale $\left(0.8^{\prime \prime} \times 3.2^{\prime \prime} \mathrm{FOV}\right)$ was used for the other companions (see Table 4). All observations were taken in ABBA nodded pairs for sky subtraction. Immediately before or after our science observations we targeted single A0V standards at a similar airmass and sky position for telluric absorption measurements.

Basic 2D image reduction, pairwise sky subtraction, and rectification into 3D cubes was carried out with version 3.2 of the OSIRIS Data Reduction Pipeline maintained by Keck Observatory. Rectification matrices derived closest in time prior to the observations were used for the pipeline processing. The science and standard spectra were extracted using aperture photometry, normalized to a common level, then mediancombined. Telluric correction was carried out with xtellcor general, part of the Spextool reduction package for IRTF/SpeX (Vacca et al. 2003; Cushing et al. 2004). Individual bandpasses were flux-calibrated with photometry from Rodriguez et al. (2012) for HD 23514 B and from Table 3 for the remaining targets. The median signal-to-noise ratio $(\mathrm{S} / \mathrm{N})$ per pixel of our (unsmoothed) OSIRIS spectra range from $25-120$.

\subsubsection{IRTF/SpeX}

We obtained moderate-resolution spectra of the wide-separation $\left(3^{\prime \prime}-8^{\prime \prime}\right)$ companions 2MASS J11240434+3808108 B, G 160-54 B, and 2MASS J02155892-0929121 C with the Infrared Telescope Facility's SpeX spectrograph in SXD mode on the nights of UT 2011 January 22, UT 2012 September 24, and UT 2014 January 18, respectively. The $0.5^{\prime \prime}$ slit width was used for all three targets, yielding a resolving power of $R \approx 1200$. We also observed the unresolved primary star PYC J11519+0731 Aab in SXD mode with a $0.8^{\prime \prime}$ slit width $(R \approx 750)$ on UT 2012 June 1. All science data were taken in a nodded ABBA pattern. A0V standards, internal flats, and arc lamps were obtained at a similar sky position to the science target. Individual spectra were extracted, median-combined, and telluric-corrected with the Spextool reduction package (Vacca et al. 2003; Cushing et al. 2004). The median $\mathrm{S} / \mathrm{N}$ per pixel of our SpeX spectra range from $30-60$.

\subsection{Optical Spectroscopy}

\subsubsection{Mayall/Ritchey-Chretien Spectrograph}

We obtained low-resolution optical spectra of most of the primary stars in our sample to provide uniform spectral classification and measure $\mathrm{H} \alpha$ line strengths. We targeted PYC J11519+0731 Aab with the Ritchey-Chretien Spectrograph (RC-Spec) using the T2KA CCD mounted on the NOAO Mayall $4 \mathrm{~m}$ telescope on 2014 May 22 UT. The $1.5^{\prime \prime} \times 98^{\prime \prime}$ slit was fixed in the north-south direction throughout the night, so we observed PYC J11519+0731 at a low airmass $(\sec z=1.1)$ near transit to minimize slit losses from differential atmospheric refraction (Filippenko 1982). We used the $6001 \mathrm{~mm}^{-1}$ BL420 grating in first order, which provided a resolving power of $R \approx$ 2600 spanning 6300-9200 $\AA$. The raw $240 \mathrm{~s}$ image was corrected for bad pixels and cosmic rays, bias-subtracted, and flat-fielded. We then extracted the spectrum by summing in the spatial direction and corrected for throughput losses using our observations of the spectrophotometric standard HZ 44. 
Table 3

Adaptive Optics Imaging Observations

\begin{tabular}{|c|c|c|c|c|c|c|c|c|c|c|}
\hline Name & $\begin{array}{l}\text { Date } \\
\text { (UT) }\end{array}$ & $\begin{array}{l}\text { Epoch } \\
\text { (UT) }\end{array}$ & $\begin{array}{l}\text { Telescope/ } \\
\text { Instrument }\end{array}$ & Filter & $\begin{array}{c}N \times \text { Coadds } \times t_{\text {exp }} \\
(\mathrm{s})\end{array}$ & $\begin{array}{c}\text { Separation } \\
\text { (mas) }\end{array}$ & $\begin{array}{l}\text { P.A. } \\
\left({ }^{\circ}\right)\end{array}$ & $\Delta \operatorname{mag}$ & $\begin{array}{l}m_{\mathrm{A}}{ }^{\mathrm{a}} \\
(\mathrm{mag})\end{array}$ & $\begin{array}{c}m_{\mathrm{B}}^{\mathrm{b}} \\
(\mathrm{mag})\end{array}$ \\
\hline 2M01225093-2439505 AB & 2013 Aug 17 & 2013.6258 & Keck II/NIRC2 & $Y$ & $14 \times 20 \times 2$ & $1448 \pm 3$ & $216.52 \pm 0.09$ & $7.6 \pm 0.3$ & $10.61 \pm 0.03$ & $18.2 \pm 0.3$ \\
\hline \multirow[t]{3}{*}{ 2M02155892-0929121 AB } & 2012 Aug 23 & 2012.6451 & Keck II/NIRC2 & $K_{S}$ & $20 \times 100 \times 0.028$ & $575.5 \pm 0.5$ & $289.98 \pm 0.03$ & $1.17 \pm 0.02$ & $7.86 \pm 0.02$ & $9.03 \pm 0.03$ \\
\hline & 2012 Oct 12 & 2012.7816 & Subaru/IRCS & $K$ & $5 \times 5 \times 0.5$ & $575 \pm 2$ & $289.9 \pm 0.5$ & $1.16 \pm 0.02$ & $7.84 \pm 0.03^{\mathrm{c}}$ & $9.00 \pm 0.03^{\mathrm{c}}$ \\
\hline & 2013 Aug 17 & 2013.6259 & Keck II/NIRC2 & $K_{S}$ & $10 \times 10 \times 0.2$ & $576.35 \pm 0.11$ & $289.351 \pm 0.011$ & $1.112 \pm 0.006$ & $7.88 \pm 0.02$ & $8.99 \pm 0.02$ \\
\hline \multirow[t]{2}{*}{ 2M02155892-0929121 AC } & 2012 Aug 23 & 2012.6451 & Keck II/NIRC2 & $K_{S}+\operatorname{cor} 600$ & $5 \times 1 \times 2.9$ & $3430 \pm 3$ & $299.4 \pm 0.2$ & $4.1 \pm 0.2$ & $7.57 \pm 0.02$ & $11.7 \pm 0.2$ \\
\hline & 2012 Oct 12 & 2012.7816 & Subaru/IRCS & K & $4 \times 5 \times 0.5$ & $3370 \pm 80$ & $299.57 \pm 0.17$ & $4.50 \pm 0.16$ & $7.54 \pm 0.03^{c}$ & $12.04 \pm 0.16^{\mathrm{c}}$ \\
\hline \multirow[t]{2}{*}{ 2M02155892-0929121 Aab } & 2012 Aug 23 & 2012.6451 & Keck II/NIRC2 & $K_{S}$ & $20 \times 100 \times 0.028$ & $42 \pm 7$ & $112 \pm 2$ & $1.2 \pm 0.3$ & $7.86 \pm 0.08$ & $9.1 \pm 0.2$ \\
\hline & 2013 Aug 17 & 2013.6259 & Keck II/NIRC2 & $K_{S}$ & $10 \times 10 \times 0.2$ & $42 \pm 7$ & $308 \pm 5$ & $1.17 \pm 0.05$ & $7.86 \pm 0.03$ & $9.03 \pm 0.04$ \\
\hline 2M02594789-0913109 АB & 2013 Aug 17 & 2013.6259 & Keck II/NIRC2 & $K_{S}$ & $10 \times 10 \times 0.2$ & $648.6 \pm 0.3$ & $320.53 \pm 0.01$ & $2.70 \pm 0.02$ & $10.22 \pm 0.02$ & $12.92 \pm 0.03$ \\
\hline \multirow[t]{2}{*}{ 1RXS J034231.8+121622 AB } & 2007 Dec 14 & 2007.9513 & Keck II/NIRC2 & $K_{S}$ & $10 \times 30 \times 1.5$ & $883.0 \pm 0.2$ & $17.58 \pm 0.09$ & $3.62 \pm 0.04$ & $9.313 \pm 0.018$ & $12.93 \pm 0.04$ \\
\hline & 2010 Aug 29 & 2010.6585 & Gemini-S/NICI & $H$ & $8 \times 1 \times 60$ & $851 \pm 3$ & $18.7 \pm 0.1$ & $3.84 \pm 0.06$ & $9.580 \pm 0.019$ & $13.42 \pm 0.06$ \\
\hline 2M05464932-0757427 AB & 2013 Jan 17 & 2013.0448 & Keck II/NIRC2 & $K_{S}$ & $10 \times 10 \times 1.0$ & $2877.3 \pm 1.2$ & $114.48 \pm 0.03$ & $3.86 \pm 0.03$ & $9.81 \pm 0.02$ & $13.67 \pm 0.04$ \\
\hline \multirow{6}{*}{ 2M06475229-2523304 AB } & 2012 Oct 13 & 2012.7847 & Subaru/IRCS & $J$ & $5 \times 5 \times 0.4$ & $1116 \pm 8$ & $28.9 \pm 0.5$ & $5.22 \pm 0.09$ & $8.36 \pm 0.02$ & $13.58 \pm 0.09$ \\
\hline & 2012 Oct 13 & 2012.7847 & Subaru/IRCS & $H$ & $4 \times 1 \times 10.0$ & $1071 \pm 7$ & $28.7 \pm 0.3$ & $4.89 \pm 0.12$ & $7.77 \pm 0.04$ & $12.66 \pm 0.13$ \\
\hline & 2012 Oct 13 & 2012.7847 & Subaru/IRCS & $K$ & $4 \times 10 \times 0.26$ & $1089 \pm 23$ & $28.8 \pm 0.2$ & $5.1 \pm 0.5$ & $7.53 \pm 0.02^{\mathrm{c}}$ & $12.6 \pm 0.5^{\mathrm{c}}$ \\
\hline & 2013 Jan 18 & 2013.0478 & Keck II/NIRC2 & $K$ & $5 \times 100 \times 0.106$ & $1086.6 \pm 1.2$ & $28.08 \pm 0.07$ & $5.538 \pm 0.012$ & $7.53 \pm 0.02^{\mathrm{c}}$ & $13.07 \pm 0.03^{\mathrm{c}}$ \\
\hline & 2014 Nov 8 & 2014.8531 & Keck II/NIRC2 & Ks & $9 \times 10 \times 0.1$ & $1088 \pm 2$ & $28.10 \pm 0.09$ & $5.33 \pm 0.16$ & $7.55 \pm 0.02$ & $12.88 \pm 0.16$ \\
\hline & 2014 Nov 8 & 2014.8531 & Keck II/NIRC2 & $K s+\operatorname{cor} 600$ & $10 \times 1 \times 30$ & $1089.9 \pm 0.7$ & $27.80 \pm 0.03$ & & $\cdots$ & \\
\hline \multirow[t]{3}{*}{ 2M08540240-3051366 AB } & 2013 Jan 18 & 2013.0478 & Keck II/NIRC2 & $J$ & $4 \times 100 \times 0.053$ & $1741 \pm 3$ & $159.08 \pm 0.03$ & $3.00 \pm 0.07$ & $9.08 \pm 0.03$ & $12.08 \pm 0.07$ \\
\hline & 2013 Jan 18 & 2013.0478 & Keck II/NIRC2 & $H$ & $4 \times 100 \times 0.053$ & $1741.2 \pm 1.3$ & $159.07 \pm 0.08$ & $2.97 \pm 0.03$ & $8.46 \pm 0.04$ & $11.43 \pm 0.05$ \\
\hline & 2013 Jan 18 & 2013.0478 & Keck II/NIRC2 & $K$ & $4 \times 100 \times 0.181$ & $1740.9 \pm 1.8$ & $159.07 \pm 0.05$ & $2.848 \pm 0.008$ & $8.142 \pm 0.03^{\mathrm{c}}$ & $10.99 \pm 0.03^{\mathrm{c}}$ \\
\hline \multirow[t]{2}{*}{$2 \mathrm{M} 11240434+3808108 \mathrm{AB}$} & 2014 Jun 13 & 2014.4463 & P60/Robo-AO & $i^{\prime}$ & $1 \times 1 \times 60$ & $8250 \pm 90$ & $131.2 \pm 0.5$ & $4.63 \pm 0.05$ & $12.554 \pm 0.001^{\mathrm{f}}$ & $17.18 \pm 0.05^{\mathrm{f}}$ \\
\hline & 2014 Jun 13 & 2014.4463 & P60/Robo-AO & $z^{\prime}$ & $1 \times 1 \times 60$ & $8270 \pm 90$ & $131.1 \pm 0.5$ & $3.90 \pm 0.03$ & $13.985 \pm 0.013^{\mathrm{f}}$ & $17.88 \pm 0.03^{\mathrm{f}}$ \\
\hline \multirow[t]{11}{*}{ PYC $11519+0731$ AabB } & 2012 May 22 & 2012.3896 & Keck II/NIRC2 & $Y$ & $11 \times 5 \times 0.5$ & $496 \pm 3$ & $110.6 \pm 0.3$ & $5.3 \pm 0.3$ & $9.31 \pm 0.03^{\mathrm{d}}$ & $14.6 \pm 0.3^{\mathrm{d}}$ \\
\hline & 2012 May 22 & 2012.3896 & Keck II/NIRC2 & $J$ & $20 \times 100 \times 0.05$ & $497 \pm 2$ & $110.4 \pm 0.2$ & $5.40 \pm 0.15$ & $8.82 \pm 0.03$ & $14.22 \pm 0.15$ \\
\hline & 2012 May 22 & 2012.3896 & Keck II/NIRC2 & $H$ & $22 \times 10 \times 0.028$ & $496.7 \pm 1.4$ & $110.4 \pm 0.2$ & $5.4 \pm 0.2$ & $8.14 \pm 0.04$ & $13.5 \pm 0.2$ \\
\hline & 2012 May 22 & 2012.3896 & Keck II/NIRC2 & $K_{S}$ & $17 \times 100 \times 0.028$ & $497.1 \pm 1.1$ & $110.46 \pm 0.09$ & $5.23 \pm 0.09$ & $7.90 \pm 0.03$ & $13.13 \pm 0.10$ \\
\hline & 2012 May 22 & 2012.3896 & Keck II/NIRC2 & $L^{\prime}$ & $10 \times 10 \times 0.11$ & $500 \pm 3$ & $110.4 \pm 0.2$ & $4.97 \pm 0.13$ & $7.80 \pm 0.11^{\mathrm{e}}$ & $12.77 \pm 0.17^{\mathrm{e}}$ \\
\hline & 2012 Jun 24 & 2012.4798 & Keck II/NIRC2 & $H$ & $20 \times 100 \times 0.028$ & $498.2 \pm 2.3$ & $110.21 \pm 0.15$ & $5.18 \pm 0.09$ & $8.14 \pm 0.04$ & $13.32 \pm 0.10$ \\
\hline & 2012 Jun 24 & 2012.4798 & Keck II/NIRC2 & $K_{S}$ & $19 \times 100 \times 0.028$ & $499.2 \pm 0.5$ & $110.30 \pm 0.10$ & $5.08 \pm 0.12$ & $7.90 \pm 0.05$ & $12.98 \pm 0.13$ \\
\hline & 2013 Jan 18 & 2013.0479 & Keck II/NIRC2 & $H+\operatorname{cor} 600$ & $20 \times 1 \times 30$ & $500.9 \pm 1.8$ & $108.76 \pm 0.05$ & $\cdots$ & $\cdots$ & $\cdots$ \\
\hline & 2013 Apr 19 & 2013.2957 & Gemini-S/NICI & $H$ & $10 \times 10 \times 1.14$ & $517 \pm 10$ & $108.3 \pm 0.5$ & $6.0 \pm 0.2$ & $8.14 \pm 0.04$ & $14.1 \pm 0.2$ \\
\hline & 2013 Apr 19 & 2013.2957 & Gemini-S/NICI & $K_{S}$ & $10 \times 10 \times 1.14$ & $511 \pm 10$ & $107.9 \pm 0.5$ & $5.4 \pm 0.2$ & $7.89 \pm 0.04$ & $13.3 \pm 0.2$ \\
\hline & 2013 Dec 18 & 2013.9628 & Keck II/NIRC2 & $K_{S}+\operatorname{cor} 600$ & $9 \times 2 \times 10$ & $514 \pm 2$ & $107.15 \pm 0.04$ & $\cdots$ & $\cdots$ & $\cdots$ \\
\hline \multirow[t]{7}{*}{ G $180-11$ AB } & 2013 Feb 04 & 2013.0949 & Keck II/NIRC2 & Y & $10 \times 10 \times 0.5$ & $1620.4 \pm 1.3$ & $254.98 \pm 0.03$ & $2.089 \pm 0.008$ & $9.60 \pm 0.02^{\mathrm{d}}$ & $11.70 \pm 0.02^{\mathrm{d}}$ \\
\hline & 2013 Feb 04 & 2013.0949 & Keck II/NIRC2 & $J$ & $10 \times 10 \times 0.2$ & $1619.9 \pm 1.2$ & $254.92 \pm 0.05$ & $2.063 \pm 0.010$ & $9.08 \pm 0.02$ & $11.14 \pm 0.02$ \\
\hline & 2013 Feb 04 & 2013.0949 & Keck II/NIRC2 & $H$ & $19 \times 10 \times 0.028$ & $1619.6 \pm 1.4$ & $254.97 \pm 0.03$ & $2.08 \pm 0.02$ & $8.41 \pm 0.03$ & $10.49 \pm 0.04$ \\
\hline & 2013 Feb 04 & 2013.0949 & Keck II/NIRC2 & $K_{S}$ & $15 \times 10 \times 0.028$ & $1620.5 \pm 1.3$ & $254.93 \pm 0.04$ & $2.06 \pm 0.03$ & $8.19 \pm 0.03$ & $10.25 \pm 0.04$ \\
\hline & 2014 Jun 13 & 2014.4463 & P60/Robo-AO & $r^{\prime}$ & $1 \times 1 \times 60$ & $1610 \pm 50$ & $253.2 \pm 1.6$ & $2.18 \pm 0.03$ & $13.544 \pm 0.004^{\mathrm{f}}$ & $15.724 \pm 0.027^{f}$ \\
\hline & 2014 Jun 13 & 2014.4463 & P60/Robo-AO & $i^{\prime}$ & $1 \times 1 \times 60$ & $1640 \pm 50$ & $253.9 \pm 1.6$ & $2.09 \pm 0.02$ & $11.749 \pm 0.003^{\mathrm{f}}$ & $13.839 \pm 0.017^{\mathrm{f}}$ \\
\hline & 2014 Jun 13 & 2014.4463 & P60/Robo-AO & $z^{\prime}$ & $1 \times 1 \times 60$ & $1630 \pm 50$ & $253.8 \pm 1.6$ & $2.03 \pm 0.02$ & $11.142 \pm 0.005^{\mathrm{f}}$ & $13.172 \pm 0.018^{f}$ \\
\hline \multirow[t]{2}{*}{$2 \mathrm{M} 15594729+4403595 \mathrm{AB}$} & 2014 Jun 13 & 2014.4463 & P60/Robo-AO & $i^{\prime}$ & $1 \times 1 \times 60$ & $5670 \pm 70$ & $284.4 \pm 0.6$ & $6.37 \pm 0.07$ & $10.433 \pm 0.001^{\mathrm{f}}$ & $16.80 \pm 0.07^{\mathrm{f}}$ \\
\hline & 2014 Jun 13 & 2014.4463 & P60/Robo-AO & $z^{\prime}$ & $1 \times 1 \times 60$ & $\cdots$ & $\cdots$ & $5.55 \pm 0.05$ & $10.147 \pm 0.001^{\mathrm{f}}$ & $15.70 \pm 0.05^{\mathrm{f}}$ \\
\hline \multirow[t]{4}{*}{ GJ 4378 Aab } & 2012 Oct 13 & 2012.7842 & Subaru/IRCS & $J$ & $4 \times 1 \times 30.0$ & $473.4 \pm 1.2$ & $348.20 \pm 0.18$ & $2.907 \pm 0.011$ & $8.71 \pm 0.02$ & $11.62 \pm 0.02$ \\
\hline & 2012 Oct 13 & 2012.7842 & Subaru/IRCS & $H$ & $5 \times 1 \times 8.0$ & $475.6 \pm 1.8$ & $348.3 \pm 0.16$ & $3.14 \pm 0.07$ & $8.13 \pm 0.03$ & $11.27 \pm 0.07$ \\
\hline & 2012 Oct 13 & 2012.7842 & Subaru/IRCS & $K$ & $5 \times 30 \times 0.145$ & $475.3 \pm 1.7$ & $348.4 \pm 0.10$ & $3.02 \pm 0.03$ & $7.93 \pm 0.05^{\mathrm{c}}$ & $10.95 \pm 0.06^{\mathrm{c}}$ \\
\hline & 2013 Jan 18 & 2013.0471 & Keck II/NIRC2 & $K$ & $13 \times 10 \times 0.053$ & $492.8 \pm 1.5$ & $347.85 \pm 0.13$ & $3.06 \pm 0.03$ & $7.92 \pm 0.05^{\mathrm{c}}$ & $10.98 \pm 0.06^{\mathrm{c}}$ \\
\hline
\end{tabular}


Table 3

(Continued)

\begin{tabular}{|c|c|c|c|c|c|c|c|c|c|c|}
\hline Name & $\begin{array}{l}\text { Date } \\
\text { (UT) }\end{array}$ & $\begin{array}{l}\text { Epoch } \\
\text { (UT) }\end{array}$ & $\begin{array}{l}\text { Telescope/ } \\
\text { Instrument }\end{array}$ & Filter & $\begin{array}{l}N \times \text { Coadds } \times t_{\exp } \\
(\mathrm{s})\end{array}$ & $\begin{array}{c}\text { Separation } \\
(\mathrm{mas})\end{array}$ & $\begin{array}{c}\text { P.A. } \\
\left({ }^{\circ}\right)\end{array}$ & $\Delta \mathrm{mag}$ & $\begin{array}{l}m_{\mathrm{A}}{ }^{\mathrm{a}} \\
(\mathrm{mag})\end{array}$ & $\begin{array}{c}m_{\mathrm{B}}^{\mathrm{b}} \\
(\mathrm{mag})\end{array}$ \\
\hline & 2013 Aug 17 & 2013.6258 & Keck II/NIRC2 & $Y$ & $10 \times 5 \times 0.5$ & $526.7 \pm 0.4$ & $347.22 \pm 0.03$ & $3.353 \pm 0.014$ & $9.21 \pm 0.02^{\mathrm{d}}$ & $12.56 \pm 0.03^{\mathrm{d}}$ \\
\hline & 2013 Aug 17 & 2013.6258 & Keck II/NIRC2 & $J$ & $10 \times 10 \times 0.05$ & $526.0 \pm 0.3$ & $347.22 \pm 0.03$ & $3.11 \pm 0.03$ & $8.70 \pm 0.02$ & $11.81 \pm 0.04$ \\
\hline & 2013 Aug 17 & 2013.6258 & Keck II/NIRC2 & $H$ & $10 \times 10 \times 0.05$ & $526.1 \pm 0.2$ & $347.18 \pm 0.03$ & $3.107 \pm 0.019$ & $8.13 \pm 0.03$ & $11.24 \pm 0.03$ \\
\hline & 2013 Aug 17 & 2013.6258 & Keck II/NIRC2 & $K_{S}$ & $15 \times 10 \times 0.1$ & $525.96 \pm 0.16$ & $347.15 \pm 0.03$ & $3.066 \pm 0.008$ & $7.87 \pm 0.03$ & $10.94 \pm 0.03$ \\
\hline & 2013 Aug 17 & 2013.6258 & Keck II/NIRC2 & $K$ & $10 \times 10 \times 0.1$ & $526.06 \pm 0.08$ & $347.194 \pm 0.018$ & $3.046 \pm 0.018$ & $7.93 \pm 0.05^{\mathrm{c}}$ & $10.97 \pm 0.05^{c}$ \\
\hline & 2013 Aug 17 & 2013.6258 & Keck II/NIRC2 & $L^{\prime}$ & $20 \times 10 \times 0.114$ & $525.9 \pm 0.6$ & $347.23 \pm 0.04$ & $2.819 \pm 0.011$ & $7.58 \pm 0.10^{\mathrm{e}}$ & $10.40 \pm 0.11^{\mathrm{e}}$ \\
\hline & 2014 Aug 04 & 2014.5901 & Keck II/NIRC2 & $K_{S}$ & $20 \times 10 \times 0.2$ & $577.2 \pm 0.4$ & $346.2 \pm 0.02$ & $3.06 \pm 0.03$ & $7.87 \pm 0.03$ & $10.93 \pm 0.04$ \\
\hline
\end{tabular}

$\checkmark \quad$ Notes.

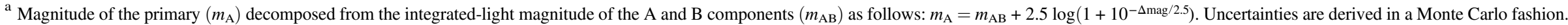

Unless otherwise noted, $J, H$, and $K_{S}$ magnitudes are from 2MASS (Skrutskie et al. 2006).

${ }^{\mathrm{b}}$ Magnitude of the companion $\left(m_{\mathrm{B}}\right)$ is computed from $\Delta$ mag and $m_{\mathrm{A}}$.

c Integrated-light $K_{S}$-band magnitude is converted to $K_{\mathrm{MKO}}$ using relations from Leggett et al. (2006).

${ }^{\mathrm{d}} Y$-band integrated-light magnitude is derived from the typical $Y-J$ color of dwarfs for the system spectral type from Rayner et al. (2009) and the integrated-light $J$-band magnitude.

${ }^{\mathrm{e}} L^{\prime}$-band integrated-light magnitude is derived from the typical $K_{S}-L^{\prime}$ color of dwarfs for the system spectral type from Golimowski et al. (2004) and the integrated-light $K_{S^{\prime}}$-band magnitude.

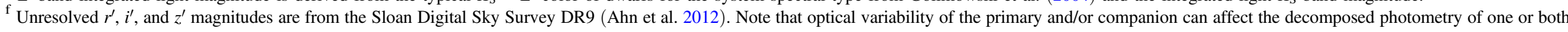
components. 
Table 4

Spectroscopic Observations

\begin{tabular}{|c|c|c|c|c|c|c|c|c|}
\hline Object & $\begin{array}{l}\text { Date } \\
\text { (UT) }\end{array}$ & $\begin{array}{l}\text { Telescope/ } \\
\text { Instrument }\end{array}$ & Filter & $\begin{array}{l}\text { Slit Width } \\
\left({ }^{\prime \prime}\right)\end{array}$ & $\begin{array}{c}\text { Plate Scale } \\
\left(\text { mas pixel }^{-1}\right)\end{array}$ & $\begin{array}{c}\text { Exp. Time } \\
\text { (minutes) }\end{array}$ & $\begin{array}{c}\text { Resolution } \\
(=\Delta \lambda \lambda)\end{array}$ & Standard $^{\mathrm{a}}$ \\
\hline 2MASS J01225093-2439505 A & 2014 Jun 28 & SOAR/Goodman & $G G 455$ & 0.46 & $\cdots$ & 5 & 1800 & LTT 6248 \\
\hline \multirow[t]{4}{*}{ 2MASS J02155892-0929121 A } & $2012 \operatorname{Dec} 28$ & Keck I/HIRES & $\ldots$ & 0.86 & $\ldots$ & 1 & 45,000 & GJ 908 \\
\hline & 2014 Jun 28 & SOAR/Goodman & $G G 455$ & 0.46 & $\ldots$ & 4 & 1800 & LTT 6248 \\
\hline & 2014 Jun 28 & SOAR/Goodman & $G G 455$ & 0.46 & $\ldots$ & 5 & 5900 & LTT 6248 \\
\hline & 2014 Dec 08 & CTIO 1.5 m/CHIRON & $\ldots$ & $\ldots$ & $\ldots$ & 45 & 28,000 & HD 42581 \\
\hline 2MASS J02155892-0929121 C & 2014 Jan 18 & IRTF/SpeX-SXD & $\ldots$ & 0.5 & 150 & 12 & 1200 & HD 13936 \\
\hline 2MASS J02594789-0913109 A & 2012 Dec 28 & Keck I/HIRES & $\ldots$ & 0.86 & $\ldots$ & 5 & 45,000 & GJ 908 \\
\hline \multirow[t]{2}{*}{ HD 23514 B } & 2013 Feb 3 & Keck I/OSIRIS & $H b b$ & $\cdots$ & 50 & 45 & 3800 & HD 23258 \\
\hline & 2013 Feb 3 & Keck I/OSIRIS & $K b b$ & $\ldots$ & 50 & 42 & 3800 & HD 23258 \\
\hline \multirow[t]{2}{*}{ G $160-54$ A } & 2007 Dec 19 & CFHT/ESPaDOnS & $\ldots$ & 0.53 & $\ldots$ & 16.7 & 68,000 & GJ 273 \\
\hline & 2012 Jan 29 & UH $2.2 \mathrm{~m} / \mathrm{SNIFS}$ & $\ldots$ & $\ldots$ & 400 & 7 & 1300 & HR 1544, G191B2B \\
\hline G $160-54 \mathrm{C}$ & 2012 Sep 24 & IRTF/SpeX-SXD & $\ldots$ & 0.5 & 150 & 24 & 1200 & HD 25792 \\
\hline \multirow[t]{3}{*}{ 2MASS J06475229-2523304 A } & 2013 Apr 30 & du Pont/Echelle & $\ldots$ & 0.75 & $\ldots$ & 10 & 45,000 & GJ 273, GJ 433 \\
\hline & 2014 Dec 3 & UH $2.2 \mathrm{~m} / \mathrm{SNIFS}$ & $\ldots$ & $\ldots$ & 400 & 3.3 & 1300 & Feige 67 \\
\hline & 2014 Dec 10 & CTIO 1.5 m/CHIRON & $\ldots$ & $\ldots$ & $\ldots$ & 15 & 28,000 & HD 217357 \\
\hline 2MASS J06475229-2523304 В & $2014 \operatorname{Dec} 8$ & Keck I/OSIRIS & $K b b$ & $\ldots$ & 20 & 18 & 3800 & HD 58886 \\
\hline 2MASS J08540240-3051366 A & 2009 Jun 13 & du Pont/Echelle & $\ldots$ & 0.75 & ... & 15 & 45,000 & GJ 908 \\
\hline \multirow[t]{2}{*}{ 2MASS J08540240-3051366 B } & $2014 \operatorname{Dec} 8$ & Keck I/OSIRIS & $H b b$ & $\ldots$ & 20 & 10 & 3800 & HD 58886 \\
\hline & $2014 \operatorname{Dec} 8$ & Keck I/OSIRIS & $K b b$ & $\ldots$ & 20 & 16 & 3800 & HD 58886 \\
\hline 2MASS J11240434+3808108 B & 2011 Jan 22 & IRTF/SpeX-SXD & $\ldots$ & 0.5 & 150 & 8 & 1200 & HD 105388 \\
\hline \multirow[t]{5}{*}{ PYC J11519+0731 Aab } & 2012 Jun 1 & IRTF/SpeX-SXD & $\ldots$ & 0.8 & 150 & 3 & 750 & HD 97585 \\
\hline & 2012 Jul 5 & Keck I/HIRES & $\ldots$ & 0.86 & $\ldots$ & 3.6 & 45,000 & $\ldots$ \\
\hline & 2013 Feb 22 & ESO-MPG $2.2 \mathrm{~m} /$ FEROS & $\ldots$ & $\ldots$ & $\ldots$ & 20 & 48,000 & $\ldots$ \\
\hline & 2014 Jun 9 & CFHT/ESPaDOnS & $\ldots$ & 0.53 & $\ldots$ & 6.7 & 68,000 & GJ 625 \\
\hline & 2014 May 22 & Mayall/RC-Spectrograph & $G G 495$ & 1.5 & 690 & 4 & 2500 & HZ 44 \\
\hline PYC J11519+0731 B & 2012 Jun 25 & Keck I/OSIRIS & $K b b$ & $\ldots$ & 20 & 40 & 3800 & HD 116960 \\
\hline \multirow[t]{3}{*}{ G $180-11$ B } & 2013 Feb 1 & Keck I/OSIRIS & $J b b$ & $\ldots$ & 50 & 6 & 3800 & q Her \\
\hline & 2013 Feb 1 & Keck I/OSIRIS & $H b b$ & $\cdots$ & 50 & 6 & 3800 & q Her \\
\hline & 2013 Feb 1 & Keck I/OSIRIS & $K b b$ & $\ldots$ & 50 & 6 & 3800 & q Her \\
\hline \multirow[t]{2}{*}{ 2MASS J15594729+4403595 A } & 2012 Feb 12 & CFHT/ESPaDOnS & $\cdots$ & 0.53 & $\cdots$ & 5 & 68,000 & GJ 628 \\
\hline & 2012 Aug 23 & UH $2.2 \mathrm{~m} / \mathrm{SNIFS}$ & $\ldots$ & $\cdots$ & 400 & 1.7 & 1300 & HR5501, EG131 \\
\hline 2MASS J15594729+4403595 B & 2012 Sep 9 & Keck II/ESI & $\cdots$ & 1.0 & 150 & 40 & 4000 & G191B2B, Feige 67 \\
\hline \multirow[t]{3}{*}{ GJ $4378 \mathrm{~A}$} & 2009 Aug 20 & du Pont/Echelle & $\cdots$ & 0.75 & $\cdots$ & 10 & 45,000 & GJ 908, GJ 699 \\
\hline & 2014 Jun 28 & SOAR/Goodman & $G G 455$ & 0.46 & $\cdots$ & 4 & 1800 & LTT 6248 \\
\hline & 2014 Jun 28 & SOAR/Goodman & $G G 455$ & 0.46 & $\cdots$ & 5 & 5900 & LTT 6248 \\
\hline \multirow[t]{2}{*}{ GJ $4378 \mathrm{Ab}$} & 2013 Jul 31 & Keck I/OSIRIS & $H b b$ & $\ldots$ & 50 & 28.5 & 3800 & HD 219833 \\
\hline & 2013 Jul 31 & Keck I/OSIRIS & $K b b$ & $\cdots$ & 50 & 11 & 3800 & HD 219833 \\
\hline \multirow[t]{3}{*}{ GJ 4379 B } & 2009 Aug 20 & du Pont/Echelle & $\ldots$ & 0.75 & $\cdots$ & 10 & 45,000 & GJ 908, GJ 699 \\
\hline & 2014 Jun 28 & SOAR/Goodman & $G G 455$ & 0.46 & $\ldots$ & 4 & 1800 & LTT 6248 \\
\hline & 2014 Jun 28 & SOAR/Goodman & $G G 455$ & 0.46 & $\ldots$ & 5 & 5900 & LTT 6248 \\
\hline
\end{tabular}

Note.

${ }^{\text {a }}$ For our high-resolution optical spectra, this refers to the RV standard. For our near-infrared and low-resolution optical spectra, this refers to telluric standards. 


\subsubsection{Southern Astrophysical Research/Goodman Spectrograph}

On UT 2014 June 28 we obtained low-resolution optical spectra of the (unresolved) primary stars 2MASS J01225093-2439505， 2MASS J02155892-0929121, GJ 4378 A, and GJ 4379 B with the Goodman High Throughput Spectrograph at the $4.1 \mathrm{~m}$ Southern Astrophysical Research (SOAR) Telescope located at Cerro Pachón, Chile. All targets were observed at low airmass $(\sec z<1.2)$ with clear sky conditions. The $0.46^{\prime \prime}$-long slit was used with a CCD read rate of $400 \mathrm{kHZ}$ and the GG455 order blocking filter. We acquired low-resolution $(R \sim 1800)$ spectra of all four targets with the $4001 \mathrm{~mm}^{-1}$ M2 grating spanning $\sim 5000-9000 \AA$. We also observed 2MASS J02155892-0929121, GJ 4378 A, and GJ $4379 \mathrm{~B}$ with a moderate-resolution setup $(R \sim 5900)$ using the $12001 \mathrm{~mm}^{-1}$ M5 grating spanning $\sim 6300-7500 \AA$. Integration times were between 240-300 s for all of our observations, and the slit was rotated to the parallactic angle to avoid slit losses from chromatic dispersion. The Goodman spectrograph suffers from strong fringing redward of $\approx 7000 \AA$, which can change from target to target from mechanical flexure. To calibrate and correct these systematics we obtained arc lamp spectra for wavelength calibration and quartz flats after each target. For the $4001 \mathrm{~mm}^{-1}$ grating we used $\mathrm{HgAr}$ lamps, and for the $12001 \mathrm{~mm}^{-1}$ grating we used $\mathrm{CuHeAr}$ lamps.

After basic image reduction entailing bad pixel correction, cosmic ray removal, bias subtraction, and flat-fielding, we removed night sky lines in the $2 \mathrm{D}$ image using the chip regions adjacent to the science spectrum in the spatial direction. The spectrum was then horizontally rectified and optimally extracted using the method from Horne (1986). For each target, fringing was corrected using the same chip region occupied by the science spectrum on the flat field. By measuring the effects of fringing in real time at each telescope position over the same pixels covered by the science data, we effectively divided out most of these artifacts in our target spectra. Finally, we corrected for throughput losses with the standard star LTT 6248 (Hamuy et al. 1992; Hamuy et al. 1994), which was observed with both grating settings.

\subsubsection{UH $2.2 \mathrm{~m} /$ SuperNova Integral Field Spectrograph}

We obtained low-resolution optical spectra of the host stars G 160-54, 2MASS J15594729+4403595, and 2MASS J06475229-2523304 with the SuperNova Integral Field Spectrograph (SNIFS; Lantz et al. 2004) at the University of Hawai'i $2.2 \mathrm{~m}$ telescope on the nights of UT 2012 January 29, UT 2012 August 23, and UT 2014 December 3. SNIFS is an optical integral field spectrograph providing low-resolution $(R \approx 1300)$ spectroscopy spanning $3200-11,000 \AA$. Several Band A-type stars and white dwarfs were targeted throughout the nights for use as spectrophotometric standards (Table 4). The short- and long-wavelength spectra were reduced, rectified into spectral cubes, and extracted in real time with the SNIFS data reduction pipeline (Aldering et al. 2006; Scalzo et al. 2010). This entailed flat-fielding, bias subtraction, wavelength calibration using arc lamps, and spectral extraction using a PSF model. Additional flux calibration and telluric correction is described in Mann et al. (2013). To summarize, we used spectrophotometric standards in conjunction with a model of the atmospheric transparency above Maunakea from Buton et al. (2012) to derive airmass and time-dependent corrections to the spectrum. The resulting flux calibration has been shown to be accurate to $1 \%-2 \%$ when compared to space-based spectra (Mann et al. 2013).

\subsubsection{Keck/HIRES}

We obtained high-resolution spectra for most of the primary stars in our sample to measure RVs, search for Li I $\lambda 6708$ absorption, and check for spectroscopic binarity. Fully convective low-mass stars and high-mass brown dwarfs burn lithium on characteristic timescales that depend on their mass; therefore the presence or absence of the lithium feature provides an independent age constraint for the system (e.g., Chabrier et al. 1996). We targeted 2MASS J021558920929121 A, 2MASS J02594789-0913109 A, and PYC J11519+0731 Aab with the High Resolution Échelle Spectrometer (HIRES; Vogt et al. 1994) on the Keck I 10 m telescope. We used the $0.861^{\prime \prime}$ slit with HIRES to give a spectral resolution of $\lambda \Delta \lambda \approx 58,000$. The detector consists of a mosaic of three $2048 \times 409615 \mu \mathrm{m}$ pixel CCDs, corresponding to a blue, green, and red chip spanning 4900-9300 $\AA$. We used the $G G 475$ filter with the red cross-disperser to maximize the throughput near the peak of an M-dwarf spectral energy distribution.

Standard reduction was performed on each stellar exposure, i.e., bias-subtraction, flat-fielding, and the correction of bad pixels. After optimal extraction, the 1D spectra were wavelength-calibrated with a Th/Ar arc taken within an hour of the stellar exposure. The resulting spectra reached $\mathrm{S} / \mathrm{N}$ of 20-50 per pixel at $7000 \AA$, depending on brightness. Each night, spectra were also taken of the RV standard GJ 908 and an A0V standard star for telluric line correction.

\subsubsection{Canada-France-Hawaii Telescope/Échelle SpectroPolarimetric Device for the Observation of Stars}

We acquired high-resolution échelle optical spectra of G16054 A, 2MASS J15594729+4403595 A, and PYC J11519+0731 Aab using the Échelle SpectroPolarimetric Device for the Observation of Stars (ESPaDOnS; Donati et al. 2006) on the $3.6 \mathrm{~m}$ Canada-France-Hawaii Telescope (CFHT). ESPaDOnS is fiberfed from the Cassegrain focus to the Coudé focus. The fiber image is projected onto a Bowen-Walraven slicer at the entrance to the spectrograph. We used the "star+sky" mode, which records the full spectrum over 40 grating orders onto a $2048 \times 4608$ pixel CCD detector covering 3700-10,400 $\AA$ with a spectral resolution $\approx 68,000$. The data were reduced using Libre Esprit, a fully automated reduction package provided for the instrument and described in detail by Donati et al. (1997, 2006). RV standards used for these targets were GJ 273, GJ 628, and GJ625.

\subsubsection{Irénee du Pont/Echelle Spectrograph}

Four of our targets were observed with the optical echelle spectrograph mounted on the $2.5 \mathrm{~m}$ du Pont telescope at Las Campanas Observatory $^{25}$ : 2MASS J06475229-2523304 A, 2MASS J08540240-3051366 A, GJ 4378 A, and GJ 4379 B. The TEK\#5 CCD with a $2 \mathrm{k}$ by $2 \mathrm{k}$ format and 24 micron pixels was employed for observations prior to December 2009. At this time, the SITe2K CCD was installed with the same format. In

\footnotetext{
25 The instrument manual can be found at http://www.lcocl/telescopesinformation/irenee-du-pont/instruments/website/echelle-spectrograph-manuals/ echelle-spectrograph-users-manual.
} 
order to reach a spectral resolution of 45,000 , we used the $0.75^{\prime \prime}$ by $4^{\prime \prime}$ slit and acquired simultaneous wavelength coverage from $\sim 3700$ to $8500 \AA$. We obtained a ThAr exposure at every pointing to avoid any flexure effects and daytime "milky" flats correct for pixel-pixel variations. In addition to a hot standard star for telluric line correction, RV standards GJ 273, GJ 433, GJ 908, and GJ 699 were also observed.

Excluding our HIRES spectrum of PYC J11519+0731 A, which is detailed in Section 3.11, our RV measurements for the HIRES, ESPaDOnS, and du Pont telescopes are carried out in a similar fashion. After correcting for the heliocentric velocity, we cross-correlated each spectral order between 7000 and $9000 \AA$ of each stellar spectrum with an RV standard of similar spectral type using IRAF's ${ }^{26}$ fxcor routine (Fitzpatrick 1993). We excluded the Ca II infrared triplet (IRT; the target stars exhibit $\mathrm{Ca}$ II emission that is not present in the RV standards) and regions of strong telluric absorption in the crosscorrelation (CC). The resulting CC function also allowed us to quickly identify double- or triple-lined spectroscopic binaries (SBs): G160-54 A, GJ 4378 A, and PYC J11519+0731 A. In nearly all cases we are able to measure RVs to better than $1 \mathrm{~km} \mathrm{~s}^{-1}$.

\subsubsection{ESO-MPG $2.2 \mathrm{~m} /$ Fiberfed Extended Range Optical Spectrograph}

As part of the ongoing CASTOFFS survey to identify young, low-mass stars in the solar neighborhood (J. E. Schlieder et al. 2015, in preparation), PYC J11519+0731 Aab was observed with the FEROS (Kaufer et al. 1999) on the ESO-MPG $2.2 \mathrm{~m}$ telescope located at La Silla, Chile, on UT 2013 February 22. Fiberfed Extended Range Optical Spectrograph (FEROS) is a fiberfed optical échelle spectrograph providing a resolution $\mathrm{R}$ $\approx 48,000$ from $\approx 3500-9200 \AA$ across 39 orders. We acquired three exposures each with integration times of $406 \mathrm{~s}$ at an airmass of 1.34, which resulted in a $\mathrm{S} / \mathrm{N}$ per pixel of $>50$ in the red orders of the median-combined spectrum. FEROS contains two $2.0^{\prime \prime}$ optical fibers separated by $2.9^{\prime}$ on sky. We observed in "object + sky" mode with one fiber on the science target and the other on sky. Data reduction was performed using the FEROS Data Reduction System (DRS) implemented within the ESO-MIDAS software package. The DRS performs flatfielding, background subtraction, bad pixel removal, order extraction (using optimal extraction), and wavelength calibration from ThAr lamp lines. The DRS then computes barycentric velocity corrections ${ }^{27}$ and rebins the individual orders to the same wavelength scale to merge them and produce a continuous spectrum. The calibrations used by the DRS (bias, flats, and a ThAr lamp spectrum) are acquired in the afternoon prior to the observations. We also observed latetype RV templates drawn from Prato et al. (2002) during observing runs in 2011 December and 2012 October for use during CC analysis (Section 3.11). Each night of FEROS observations also includes observations of at least one RV standard drawn from the lists of Chubak et al. (2012) or Nidever et al. (2002) for internal calibration checks. The RV

\footnotetext{
${ }^{26}$ IRAF is distributed by the National Optical Astronomy Observatories, which is operated by the Association of Universities for Research in Astronomy, Inc. (AURA) under cooperative agreement with the National Science Foundation.

27 Müller et al. (2013) show that the barycentric correction computed by the FEROS-DRS is only accurate to $\sim 100 \mathrm{~m} \mathrm{~s}^{-1}$ because it does not take into account coordinate precession. This small systematic error is not significant for our analysis, which achieves an RV precision of $\sim 1 \mathrm{~km} \mathrm{~s}^{-1}$.
}

templates and standards were reduced in the same way as the science target.

\subsubsection{CTIO-SMARTS $1.5 \mathrm{~m} / \mathrm{CHIRON}$}

We obtained high-resolution $(R \approx 28,000)$ optical spectra of 2MASS J02155892-0929121 A and 2MASS J064752292523304 A on the nights of UT 2014 December 8 and UT 2014 December 10, respectively, with the CHIRON echelle spectrograph (Tokovinin et al. 2013) at the CTIO $1.5 \mathrm{~m}$ telescope operated by the SMARTS Consortium at Cerro Tololo, Chile. CHIRON is an extremely stable fiberfed instrument built for high-precision $\left(\approx 1 \mathrm{~m} \mathrm{~s}^{-1}\right) \mathrm{RV}$ exoplanet searches. Each spectrum covers the 4500-8900 A spanning 62 orders. All observations are carried out in queue mode (Brewer et al. 2014) and are reduced in an automated fashion that includes bias subtraction, flat-fielding, optimal spectral extraction, and wavelength calibration of each order using a ThAr exposure acquired after each target.

We measure RVs for both targets by cross-correlating the science spectra with RV standards from Chubak et al. (2012) that have similar spectral types and were also observed using the same setup with CHIRON. Each order is first normalized, and the science and standard spectra are then linearly interpolated onto a common wavelength grid. After applying a barycentric velocity correction to each spectrum, each order is cross-correlated separately, and the RV offset was determined by fitting a Gaussian to the cross-correlation function. The final $\mathrm{RVs}$ are the mean from 10 orders spanning 6120-6860 , which were chosen to avoid telluric absorption features. Our adopted uncertainties incorporate both random errors (the standard deviation from the 10 orders) and an estimated systematic error of $0.5 \mathrm{~km} \mathrm{~s}^{-1}$ (measured by cross-correlating RV standards with each other) added in quadrature.

\subsubsection{Keck/Echellette Spectrograph and Imager}

The wide separation of 2MASS J15594729+4403595 B from its host star $\left(5.6^{\prime \prime}\right)$ makes it amenable to spectroscopy without the need for AO correction. We observed 2MASS J15594729 +4403595 B using the Echellette Spectrograph and Imager (ESI; Sheinis et al. 2002) on Keck II on UT 2012 September 9 under near-photometric conditions. We used the $1.0^{\prime \prime}$ slit, which yielded a spectral resolution of $R \approx 4000$ and wavelength coverage from $3000-10,000 \AA$. The slit was aligned perpendicular to the star-companion position angle to avoid contamination from the primary. We obtained three separate exposures, each $800 \mathrm{~s}$, which yielded a $\mathrm{S} / \mathrm{N}$ per pixel of $\approx 60$ redward of $7500 \AA$. Observations of the G191B2B and Feige 67 spectrophotometric standards were taken throughout the night.

We reduced the data using the ESIRedux package (Prochaska et al. 2003; Bochanski et al. 2009). ${ }^{28}$ ESIRedux performs bias, flat, and dark correction as well as extraction of the two-dimensional data into a one-dimensional spectrum. ESIRedux wavelength-calibrates the data using $\mathrm{HgNe}$ and $\mathrm{CuAr}$ arcs (obtained at the start of the night) to construct a twodimensional wavelength map. The orders are combined into a single spectrum, and an approximate flux calibration is applied (based on archived calibration data), which we improve upon using the observed spectrophotometric standards and a model

\footnotetext{
${ }^{28}$ http://www2.keck.hawaii.edu/inst/esi/ESIRedux/index.html
} 
of the atmosphere above Maunakea (Buton et al. 2012; Mann et al. 2013).

\section{TARGETS}

\subsection{MASS J01225093-2439505 AB}

2MASS J01225093-2439505 B is a young mid-L dwarf companion identified by Bowler et al. (2013) during the PALMS high-contrast imaging planet search. Located at $1.5^{\prime \prime}$ $(\approx 52 \mathrm{AU})$ from the young active mid-M-dwarf 2MASS J01225093-2439505 A, 2MASS J01225093-2439505 B possesses red near-IR colors and an angular $H$-band spectral morphology, hallmarks of a dusty photosphere and low surface gravity, respectively. Our low-resolution optical spectrum of the primary from the SOAR/Goodman Spectrograph is shown in Figure 1. We find a spectral type of M4.0 \pm 0.5 from visual and index-based classification with the IDLbased classification package Hammer (Table 5; Covey et al. 2007), which is slightly later than the M3.5 V classification from Riaz et al. (2006). Malo et al. (2013) found the system probably belongs to the $\approx 120 \mathrm{Myr} \mathrm{AB}$ Dor moving group, which was bolstered by Bowler et al. based on an RV measurement $\left(9.6 \pm 0.7 \mathrm{~km} \mathrm{~s}^{-1}\right)$ and the photometric distance $(36 \pm 4 \mathrm{pc})$. At this age, the companion has a luminosity that corresponds to both $\approx 13$ and $\approx 25 M_{\text {Jup }}$ masses based on substellar evolutionary models, which overlap in this region because of delayed onset of deuterium burning at lower masses (Bowler et al. 2013). More recently, Gagné et al. (2014) suggested this system may belong to the $15-25 \mathrm{Myr} \beta$ Pic moving group using the RV of $11.4 \pm 0.2 \mathrm{~km} \mathrm{~s}^{-1}$ from Malo et al. (2014), in which case the companion mass is as low as $\approx 6 M_{\text {Jup }}$. A parallactic distance is needed to unambiguously distinguish these cases. Note that the RVs from Bowler et al. and Malo et al. differ by $2.5 \sigma$, indicating the primary could be a single-line spectroscopic binary (SB1). The Radial Velocity Experiment (RAVE; Kordopatis 2013) found a slightly larger higher RV of $12.3 \pm 2.5 \mathrm{~km} \mathrm{~s}^{-1}$, which is formally consistent with the other two measurements.

On UT 2013 August 17 we obtained the first $Y$-band images of 2MASS J01225093-2439505 B with NIRC2 (Figure 2). Our astrometry is consistent with measurements between 20122013 from Bowler et al. Based on the typical $Y-J$ color of an M4.0 V star from Rayner et al. (2009), our $Y$-band contrast of $7.6 \pm 0.3 \mathrm{mag}$ implies an apparent magnitude of $Y=18.2 \pm 0.3$ mag (Table 3), an absolute magnitude of $M_{Y}=15.4 \pm 0.3 \mathrm{mag}$, and a $Y-J$ color of $1.5 \pm 0.3 \mathrm{mag}$. The relationship between $M_{Y}$ and spectral type has not yet been mapped for young brown dwarfs and giant planets, but compared to old field objects from Dupuy \& Liu (2012), our new measurement best corresponds to L5-L7 objects. This is in good agreement with our classification of L4-L6 based on $H$ - and $K$-band spectroscopy from Bowler et al. (2013).

\subsection{MASS J02155892-0929121 AabBC}

Riaz et al. (2006) classify 2MASS J02155892-0929121 as an M2.5 star displaying $\mathrm{H} \alpha$ emission $(\mathrm{EW}=-6.9 \AA)$ in their catalog of ROSAT-detected M dwarfs. We find a slightly later type of M3.5 with our Goodman spectrum (Table 5; Figures 1 and 3) but a similar $\mathrm{H} \alpha$ line strength of $-5.6 \AA$. Bergfors et al. (2010) resolved this object into three components in a single epoch of imaging in 2008 as part of the AstraLux Lucky Imaging survey: a close stellar companion at $0.6^{\prime \prime}$ ("B") and a fainter component at 3.5 " with red optical colors ("C"), which Janson et al. (2012) estimated has a spectral type of M8.

Based on its activity and proper motion, Malo et al. (2013) suggest this system is a possible member of the Columba, $\beta$ Pic, or Tuc-Hor moving groups. Kraus et al. (2014b) also identify it as a candidate Tuc-Hor member, although their measured RV $\left(10.1 \pm 0.6 \mathrm{~km} \mathrm{~s}^{-1}\right)$ disagrees with the expected value assuming group membership by $5.2 \mathrm{~km} \mathrm{~s}^{-1}$. Three additional epochs were presented by Malo et al. (2014): $0.5 \pm 0.3 \mathrm{~km} \mathrm{~s}^{-1}$ and $-0.6 \pm 0.3 \mathrm{~km} \mathrm{~s}^{-1}$ in 2010 and $8.3 \pm 0.3 \mathrm{~km} \mathrm{~s}^{-1}$ in 2012. A consistent but less precise RV of $0.6 \pm 5 \mathrm{~km} \mathrm{~s}^{-1}$ was measured by the RAVE survey in 2007 (Kordopatis 2013). These RV variations indicate the primary is an SB1 (Table 6).

We measure RVs of $6.2 \pm 0.4 \mathrm{~km} \mathrm{~s}^{-1}$ and $8.3 \pm 0.6 \mathrm{~km} \mathrm{~s}^{-1}$ for 2MASS J02155892-0929121 from spectra obtained in 2012 December and 2014 December with Keck/HIRES and SMARTS $1.5 \mathrm{~m} / \mathrm{CHIRON}$. Our Keck/HIRES measurement agrees well with the predicted RV of $4.5 \pm 1.4 \mathrm{~km} \mathrm{~s}^{-1}$ for TucHor membership from Malo et al. (2013). Based on this RV, the web-based Bayesian tool BANYAN II to compute YMG membership probabilities ${ }^{29}$ by Gagné et al. (2014) returns a 99.5\% likelihood that this system belongs to the Tuc-Hor moving group. The weighted mean RV $\left(4.0 \pm 0.15 \mathrm{~km} \mathrm{~s}^{-1}\right)$ is also consistent with the predicted value for Tuc-Hor. However, the changing RVs spanning 2010-2014 mean that more spectroscopic monitoring will be needed to derive a systemic $\mathrm{RV}$ and unambiguously determine its group membership.

We imaged 2MASS J02155892-0929121 three times between 2012-2013 with Subaru/IRCS and Keck/NIRC2 (Figure 4). We confirm the B and C components are comoving with the primary (Figure 5) and detect orbital motion between our epochs and those in 2008 from Bergfors et al. (2010). For our 2013 August data with NIRC2, only the central $198 \times 248$ pixel $^{2}\left(1.97^{\prime \prime} \times 2.47^{\prime \prime}\right)$ region of the detector encompassing the $\mathrm{AB}$ components was read out, so we do not have astrometry of the $\mathrm{C}$ component at that epoch.

\subsubsection{Resolved AO Imaging of 2MASS J02155892-0929121 AabB}

From visual inspection of our NIRC2 images in August 2012 and August 2013 we found that the A component appears slightly elongated compared to B at both epochs, but in the opposite directions (SE in 2012 and NE in 2013). The separation between $\mathrm{A}$ and $\mathrm{B}$ is close enough $\left(0.6^{\prime \prime}\right)$ that isoplanatic effects should be small, so any differences in the PSF shape are probably caused by a marginally resolved closein companion.

Using a $0.4^{\prime \prime} \times 0.4^{\prime \prime}$ region surrounding 2MASS J02155892-0929121 B as a reference PSF, we jointly fit two identical PSF templates to the A component with the NelderMead ("Amoeba") downhill-simplex minimization technique (Nelder \& Mead 1965; Press et al. 2007). Seven parameters were allowed to vary: an $x$-position, $y$-position, and multiplicative scale factor for each PSF as well as an overall additive amplitude offset. Each image was fit separately (20 in 2012 and 10 in 2013), and the resulting coadded frames are shown in Figure 6. In each case, subtracting the primary reveals a clear image of a companion and its first Airy ring. The joint fits provide excellent matches to the data as demonstrated by the low rms residuals of $\approx 8 \mathrm{DN}(70 \mathrm{DN})$ compared to a peak flux

\footnotetext{
${ }^{29}$ http://www.astro.umontreal.ca/ gagne/banyanII.php
} 
Table 5

$\mathrm{H} \alpha$ Emission and Optical Spectral Classification

\begin{tabular}{|c|c|c|c|c|c|c|}
\hline Name & $\begin{array}{c}\mathrm{H} \alpha \mathrm{EW}^{\mathrm{a}} \\
(\AA)\end{array}$ & $\begin{array}{l}\text { TiO5 } \\
\text { Index }\end{array}$ & $\begin{array}{l}\mathrm{CaH} 1 \\
\text { Index }\end{array}$ & $\begin{array}{l}\mathrm{CaH} 2 \\
\text { Index }\end{array}$ & $\begin{array}{l}\mathrm{CaH} 3 \\
\text { Index }\end{array}$ & $\begin{array}{c}\text { Adopted SpT } \\
( \pm 0.5)\end{array}$ \\
\hline 2MASS J01225093-2439505 A & -5.0 & 0.417 & 0.783 & 0.424 & 0.677 & M4.0 \\
\hline 2MASS J02155892-0929121 Aab & -5.6 & 0.482 & 0.810 & 0.485 & 0.716 & M3.5 \\
\hline 2MASS J06475229-2523304 Aab & -0.3 & 0.890 & 1.009 & 0.950 & 0.952 & K7.0 \\
\hline PYC J11519+0731 Aab & -2.8 & 0.594 & 0.866 & 0.612 & 0.814 & M2.0 \\
\hline 2MASS J15594729+4403595 A & -2.3 & 0.685 & 0.877 & 0.648 & 0.821 & M1.5 \\
\hline GJ 4379 B & 0.1 & 0.457 & 0.801 & 0.459 & 0.725 & M3.5 \\
\hline
\end{tabular}

Note.

${ }^{\mathrm{a}}$ Negative values indicate emission.

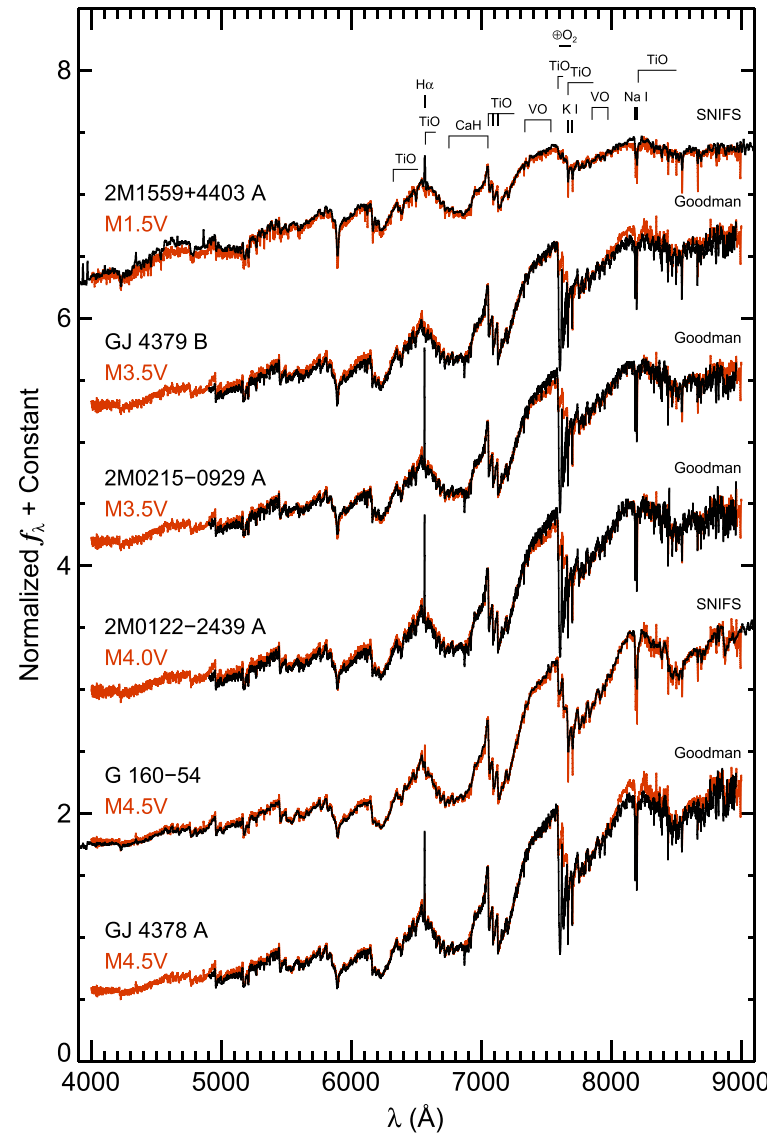

Figure 1. Low-resolution SOAR/Goodman $(R \sim 1800)$ and UH2.2 m/SNIFS $(R \sim 1300)$ optical spectra of host stars to ultracool companions. Red templates from Bochanski et al. (2007) show the best matches based on visual and indexbased classification with Hammer (Table 5; Covey et al. 2007). Half-integer subtypes are created by averaging two templates together. All spectra are normalized between $7200-7400 \AA$ and offset by a constant. Although these spectra originate from instruments with different resolving powers and wavelength spans, our spectral types agree with those in the literature (to within \pm 0.5 subtypes) for nearly all stars that have been previously classified.

of $\approx 1600 \mathrm{DN}(14,000 \mathrm{DN})$ in our 2012 (2013) data. The median and standard deviation of the separations, position angles, and $K_{S}$-band flux ratios are $42 \pm 7$ mas, $112 \pm 2^{\circ}$, and $1.2 \pm 0.3 \mathrm{mag}$ for our 2012 data set. For our 2013 data we measure $42 \pm 7$ mas, $308 \pm 5^{\circ}$, and $1.17 \pm 0.05 \mathrm{mag}$.
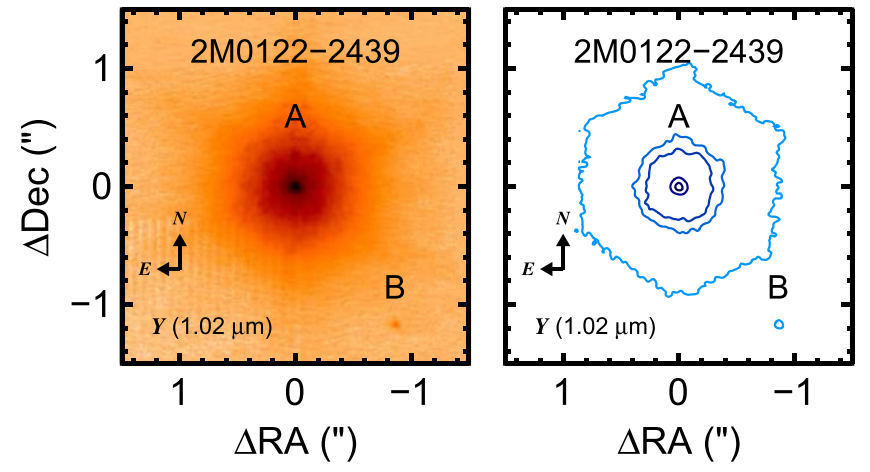

Figure 2. Keck/NIRC2 $Y$-band image of 2MASS J01225093-2439505 B. Contours represent $0.03 \%, 0.5 \%, 1 \%, 10 \%$, and $50 \%$ of the peak flux after convolution with a Gaussian kernel with a FWHM equal to that of the image PSF. North is up, and east is left.

Resolving the very close binary 2MASS J02155892$0929121 \mathrm{Aab}$ at the same separation but with a nearly $180^{\circ}$ change in P.A. suggests an orbital period of $\sim 2$ years (or shorter aliases). We perform a simple test to verify whether the amplitudes of the RV variations are plausibly caused by this companion. Based on the empirically derived relations in Boyajian et al. (2012), the M3.5 spectral type of the Aa component corresponds to an effective temperature of $\approx 3300 \mathrm{~K}$. At an age of $40 \mathrm{Myr}$, this implies a mass of $\approx 0.2$ $M_{\odot}$ for the primary (Aa) and $\approx 0.09 M_{\odot}$ for the companion $(\mathrm{Ab})$ using the measured $K$-band flux ratio and the evolutionary models of Baraffe et al. (1998). Assuming a semimajor axis of $\approx 1 \mathrm{AU}$, which roughly corresponds to the observed separation of the pair, a circular orbit, and an inclination of $90^{\circ}$, the induced velocity semiamplitude is $\approx 5.0 \mathrm{~km} \mathrm{~s}^{-1}$. This is in good agreement with the maximum total RV amplitude change of $\approx 11 \mathrm{~km} \mathrm{~s}^{-1}$ from random RV sampling over the past decade.

There is no parallactic distance for this system, but spectrophotometric distance estimates to 2MASS J02155892$0929121 \mathrm{C}$ (see below) and the unresolved primary (Riaz et al. 2006; Kraus et al. 2014b) imply distances of $\approx 20-30$ pc. These correspond to physical separations of $\approx 1 \mathrm{AU}$ for Aab. Fortunately, the nearby components 2MASS J02155892$0929121 \mathrm{~B}$ and C (see below) offer excellent opportunities to measure individual dynamical masses of pre-main-sequence $\mathrm{M}$ dwarfs using absolute astrometry of the system as opposed to a total mass with relative astrometry. 


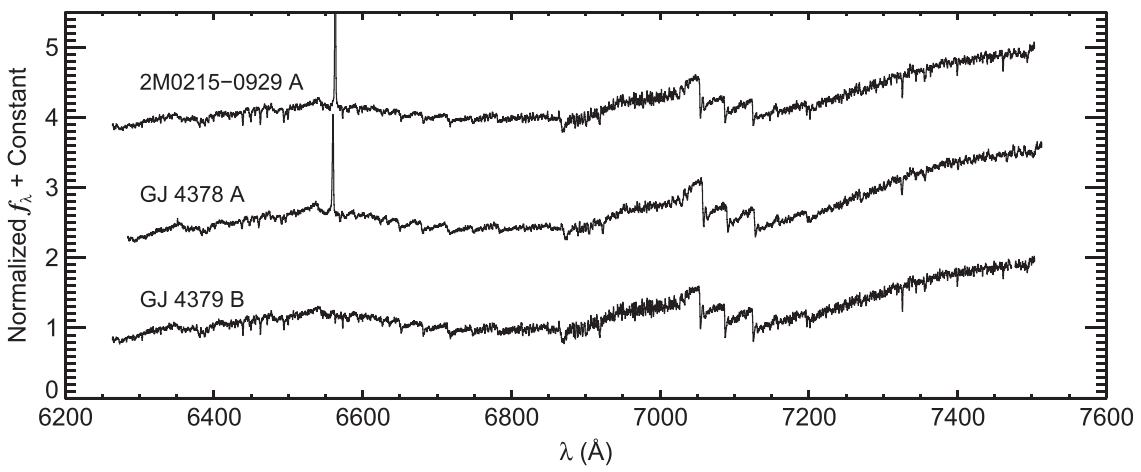

Figure 3. Moderate-resolution $(R \sim 5900)$ spectra of 2MASS J02155892-0929121 A, GJ 4378 A, and GJ 4379 B from the SOAR/Goodman spectrograph. None of the targets show signs of $\mathrm{Li}$ I $\lambda 6708$ absorption. All spectra are normalized between 7200-7400 $\AA$ and offset by a constant.

Table 6

Radial Velocities

\begin{tabular}{|c|c|c|c|c|}
\hline Name & Component & $\begin{array}{c}\text { UT Date } \\
\text { (YYYY-MM-DD) }\end{array}$ & $\begin{array}{c}\mathrm{RV} \\
\mathrm{km} \mathrm{s}^{-1}\end{array}$ & Reference \\
\hline \multirow[t]{3}{*}{ 2MASS J01225093-2439505 } & A & 2010 Sep 24 & $11.4 \pm 0.2$ & M14 \\
\hline & A & 2011 Aug 24 & $12.3 \pm 2.5$ & K13 \\
\hline & A & $2012 \operatorname{Dec} 28$ & $9.6 \pm 0.7$ & B13 \\
\hline \multirow[t]{7}{*}{ 2MASS J02155892-0929121 } & $\mathrm{Aa}$ & 2007 Nov 08 & $0.6 \pm 5.0$ & K13 \\
\hline & Aa & $2010 \mathrm{Jul} 26$ & $0.5 \pm 0.3$ & M14 \\
\hline & $\mathrm{Aa}$ & 2010 Sep 19 & $-0.6 \pm 0.3$ & M14 \\
\hline & Aa & 2012 Feb 11 & $8.3 \pm 0.3$ & M14 \\
\hline & Aa & $2012 \mathrm{Jul} 18$ & $10.1 \pm 0.6$ & K14 \\
\hline & Aa & $2012 \operatorname{Dec} 28$ & $6.2 \pm 0.4$ & TW \\
\hline & Aa & 2014 Dec 08 & $8.3 \pm 0.6$ & TW \\
\hline 2MASS J02594789-0913109 & A & $2012 \operatorname{Dec} 28$ & $4.9 \pm 0.5$ & TW \\
\hline 1RXS J034231.8+121622 & $\mathrm{A}$ & 2005 Dec 21 & $35.4 \pm 0.4$ & S12 \\
\hline \multirow[t]{5}{*}{ 2MASS J06475229-2523304 } & $\mathrm{Aa}$ & $\ldots$ & -56.5 & T06 \\
\hline & Aa & 2009 Feb 28 & $-67 \pm 6$ & K13 \\
\hline & $\mathrm{Aa}$ & 2011 Sep 14 & $-24.4 \pm 0.8$ & M14 \\
\hline & $\mathrm{Aa}$ & 2013 Apr 30 & $-2.1 \pm 0.9$ & TW \\
\hline & $\mathrm{Aa}$ & 2014 Dec 10 & $-5.5 \pm 0.8$ & $\mathrm{TW}$ \\
\hline 2MASS J08540240-3051366 & $\mathrm{A}$ & 2009 Jun 13 & $44.5 \pm 0.6$ & TW \\
\hline \multirow[t]{2}{*}{ 2MASS J11240434+3808108 } & A & 2006 May 11 & $-11.5 \pm 0.5$ & $\mathrm{~S} 12$ \\
\hline & $\mathrm{B}$ & $\ldots$ & $-14 \pm 3$ & R09 \\
\hline \multirow[t]{7}{*}{ PYC J11519+0731 } & Aa & $2012 \mathrm{Jul} 05$ & $50.9 \pm 1.0$ & TW \\
\hline & $\mathrm{Aa}$ & 2013 Feb 22 & $14.7 \pm 1.0$ & $\mathrm{TW}$ \\
\hline & $\mathrm{Aa}$ & 2014 Jun 09 & $69.5 \pm 1.0$ & TW \\
\hline & $\mathrm{Ab}$ & $2012 \mathrm{Jul} 05$ & $-51.9 \pm 1.0$ & TW \\
\hline & $\mathrm{Ab}$ & 2013 Feb 22 & $-18.2 \pm 1.0$ & TW \\
\hline & $\mathrm{Ab}$ & 2014 Jun 09 & $-75.5 \pm 1.0$ & TW \\
\hline & $\gamma$ & $\ldots$ & $-0.3 \pm 1.0$ & TW \\
\hline G $180-11$ & A & 2006 Aug 13 & $-15.5 \pm 0.7$ & S12 \\
\hline \multirow[t]{2}{*}{ 2MASS J15594729+4403595 } & A & 2013 Mar 03 & $-15.8 \pm 0.5$ & M14 \\
\hline & A & 2012 Feb 12 & $-19.6 \pm 0.6$ & TW \\
\hline \multirow[t]{2}{*}{ GJ $4378 \mathrm{~A}$} & a1 & 2009 Aug 20 & $-7.0 \pm 0.8$ & TW \\
\hline & $\mathrm{a} 2$ & 2009 Aug 20 & $49.2 \pm 1.1$ & $\mathrm{TW}$ \\
\hline GJ 4379 B & $\cdots$ & 2009 Aug 20 & $20.6 \pm 0.4$ & TW \\
\hline
\end{tabular}

References. K13—Kordopatis (2013), K14—Kraus et al. (2014b), M14—Malo et al. (2014), R09—Reiners \& Basri (2009), S12—Shkolnik et al. (2012), T06— Torres et al. (2006), TW-this work.

\subsubsection{The Ultracool Companion 2MASS J02155892-0929121 C}

Our 0.8-2.5 $\mu \mathrm{m}$ spectrum of 2MASS J02155892-0929121 C is shown in Figure 7. Overall, the NIR spectral energy distribution (SED) agrees fairly well with the M7 field template. Individual bandpasses are most similar to $\mathrm{M} 7-\mathrm{M} 8$ objects. The $\mathrm{H}_{2} \mathrm{O}, \mathrm{H}_{2} \mathrm{O}-1$, and $\mathrm{H}_{2} \mathrm{O}-2$ gravity-insensitive indexbased classifications from Allers \& Liu (2013), which were originally defined by Allers et al. (2007) and Slesnick et al. (2004), give spectral types of M6.6 $\pm 1.7, \mathrm{M} 7.0 \pm 2.3$, and M6.7 \pm 2.2 with a weighted mean of M6.7 \pm 1.2 . Altogether we adopt a final NIR spectral type of M7 \pm 1 . Of note are the shallow 1.244 and $1.252 \mu \mathrm{m} \mathrm{K}$ and $1.2 \mu \mathrm{m} \mathrm{FeH}$ features in $J$ band, hallmark signatures of low surface gravity and youth $(\lesssim 200 \mathrm{Myr}$ ) in brown dwarfs (e.g., McGovern et al. 2004). We confirm this quantitatively using the indices from Allers \& Liu 


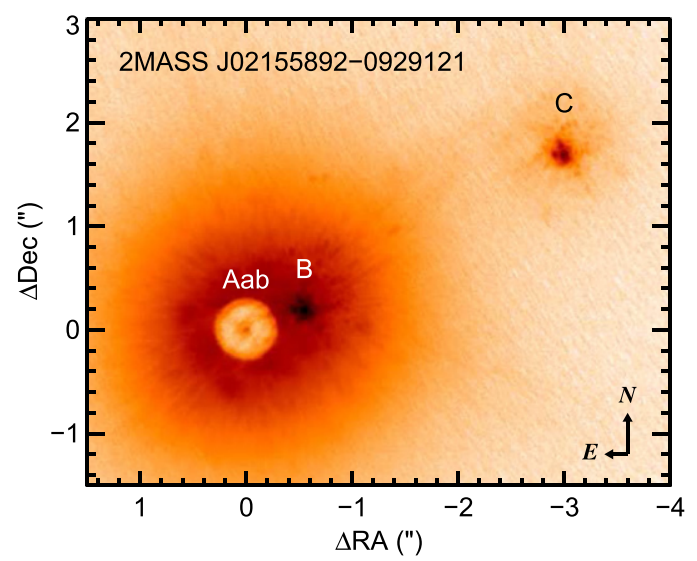

Figure 4. Keck/NIRC2 $K_{S}$-band coadded image of the young 2MASS J02155892-0929121 system. The primary ("Aab") is positioned behind the 600 mas partly opaque coronagraph. The stellar ("B") and brown dwarf ("C") companions are located at separations $0.6^{\prime \prime}$ and $3.5^{\prime \prime}$, respectively. North is up, and east is left.

(2013), which yield an intermediate-gravity (INT-G) classification for 2MASS J02155892-0929121 C (Table 8). This is strong evidence independent of kinematics that the system is quite young-likely $\approx 30-150 \mathrm{Myr}$-implying that 2MASS J02155892-0929121 C is indeed substellar.

The $K$-band photometric distance to 2MASS J02155892$0929121 \mathrm{C}$ is $29 \pm 5 \mathrm{pc}$ using the field relations from Dupuy \& Liu (2012). Because the system is young, this distance is probably underestimated. Nevertheless, adopting the $K$-band bolometric correction from Liu et al. (2010a) gives a luminosity of $\log \left(L / L_{*}\right)=-3.23 \pm 0.16$ dex, implying a mass of $42 \pm 15 M_{\text {Jup }}$ based on cooling models from Burrows et al.
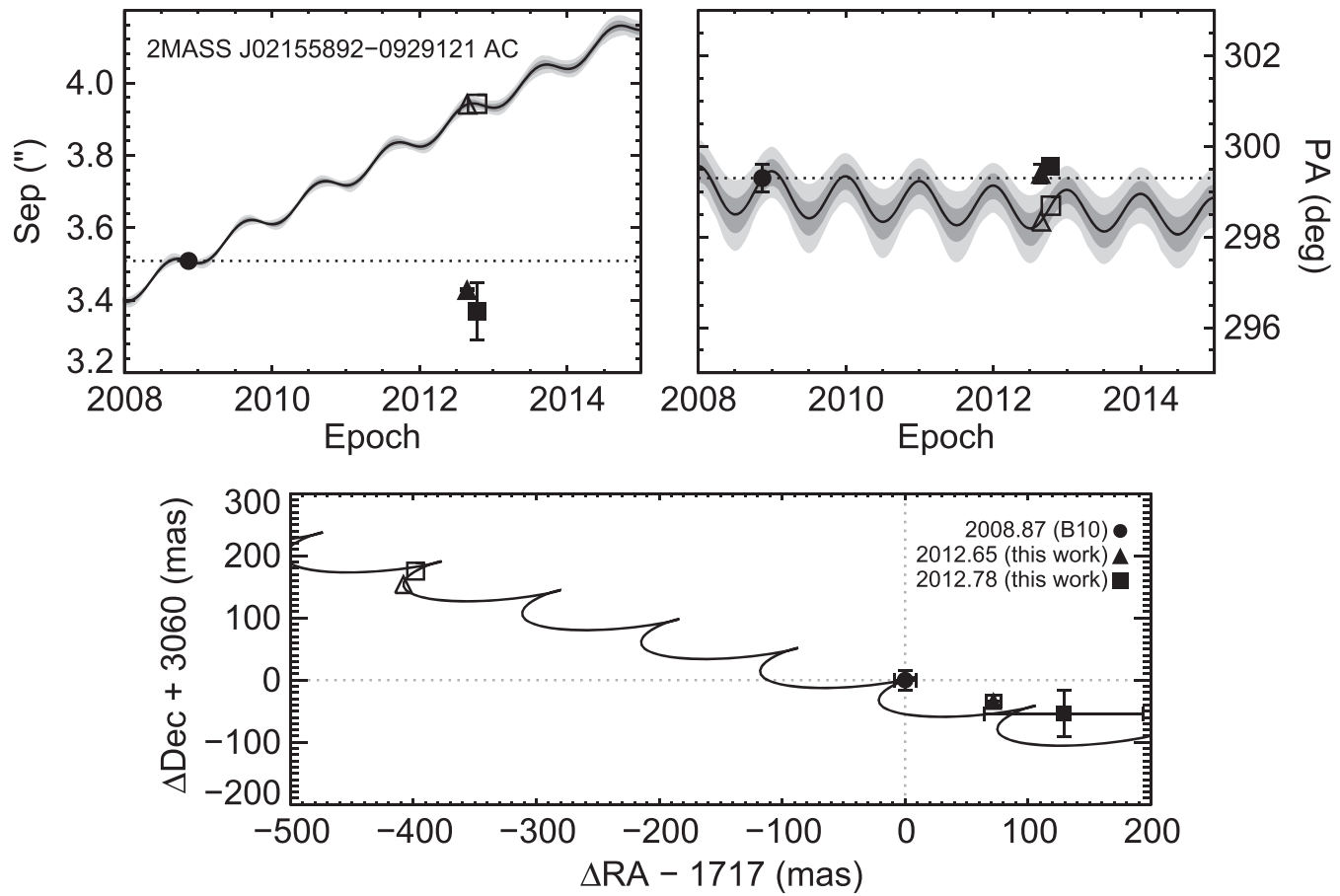

Figure 5. Astrometry of the substellar companion 2MASS J02155892-0929121 C between 2008-2014 relative to the primary, which itself is a close 40 mas visual binary. The solid curve shows the expected relative astrometry of a background object; the gray shaded regions show $1 \sigma$ and $2 \sigma$ confidence intervals. Open symbols represent the predicted astrometry for a stationary object at the epochs of the observations. Compared to astrometry from Bergfors et al. (2010), 2MASS J02155892-0929121 C is clearly comoving. There is some evidence of orbital motion in the system, which is likely caused by motion from the Aab primary, Aab-B component, and/or between the Aab-C components of this quadruple system. 

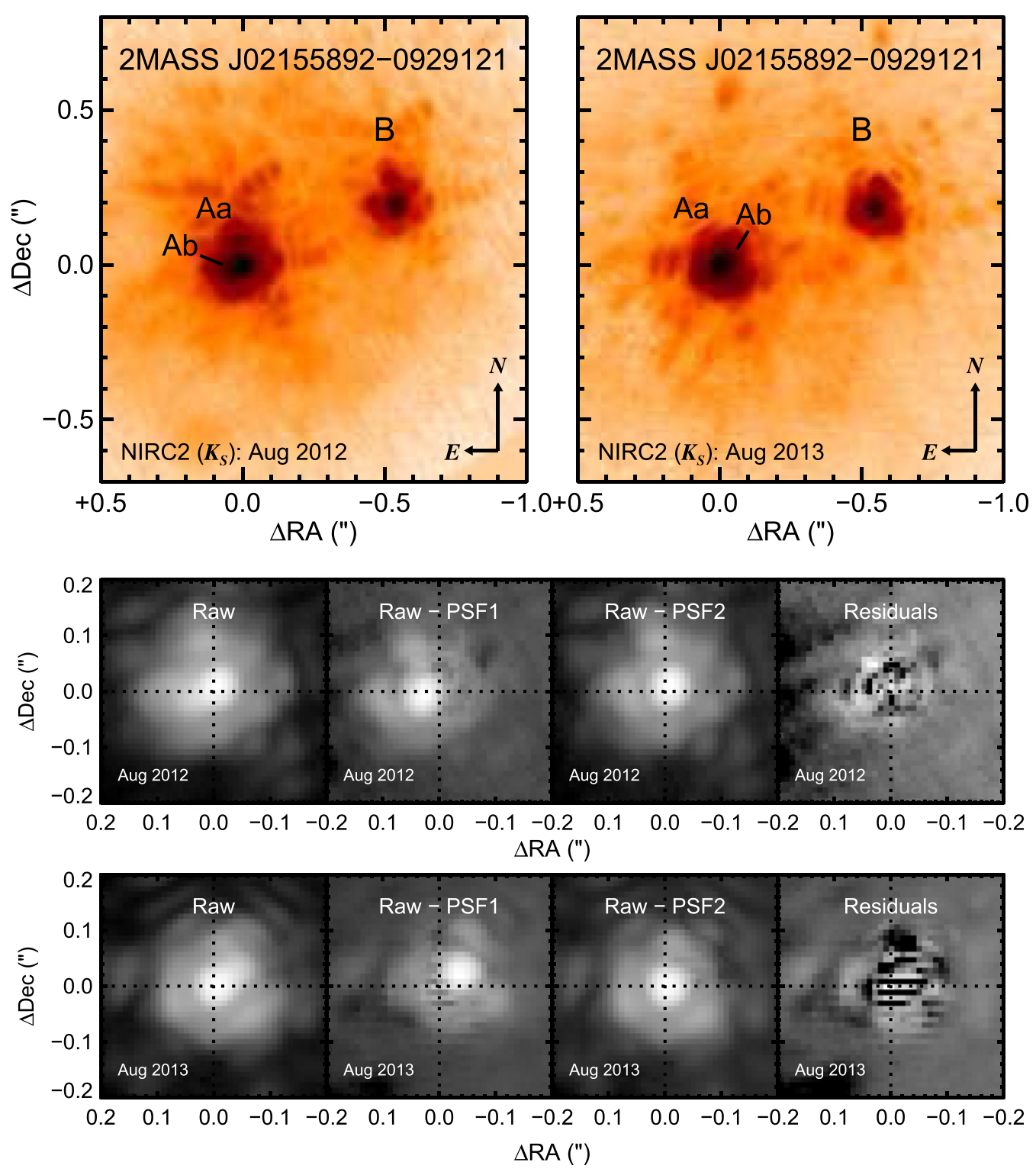

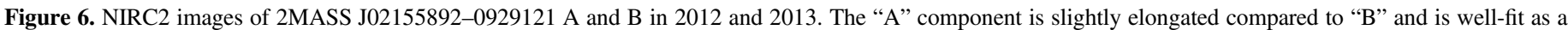

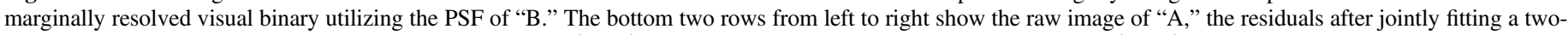

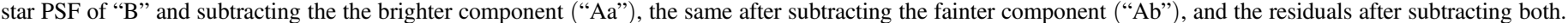
In 2012 August, 2MASS J02155892-0929121 Ab is $\approx 40$ mas southeast of Aa, and in 2013 August it is $\approx 40$ mas northwest of Aa.

spectroscopy. Based on the age and luminosity of the system, Bowler et al. inferred a mass of $35 \pm 8 M_{\text {Jup }}$ for $1 \mathrm{RXS}$ J034231.8+121622 B.

Here we present additional astrometry of 1RXS J034231.8 +121622 AB between 2007 and 2013 with Keck/NIRC2 and Gemini-S/NICI. Combined with published astrometry (Table 7) there is clear orbital motion in the system. Although the coverage is quite limited, only a subset of Keplerian orbits are consistent with the observed astrometry. Our first attempt to constrain the orbital parameters and total mass of the system with orbit fitting is described below.

The orbital elements are calculated by fitting the resolved astrometric observations to a Keplerian orbit using emcee (Foreman-Mackey et al. 2013), an implementation of the affine-invariant MCMC ensemble sampler of Goodman \& Weare (2010). Our model has eight free parameters: $\sqrt{e} \cos \omega, \sqrt{e} \sin \omega$, the time of periapsis, $\log ($ period $), \log$ (total mass), inclination, the position angle of the ascending node, and distance. For the distance, we apply a prior following the parallax measured by Dittmann et al. (2014). At each step, we forward model the orbits of the two components, calculating their relative projected positions on the sky as viewed from the Earth at the time of each astrometric observation and determining the likelihood of these orbital elements (Figure 8). We find that even though observations cover only $1.5 \%$ of the orbit, we are able to constrain the orbital period to $12 \%$ and the total mass to $18 \%$.

The median and $68 \%$ confidence intervals of the posteriors are $411 \pm 51$ years for the orbital period, $0.16 \pm 0.07$ for the eccentricity, and $0.152 \pm 0.027 M_{\odot}$ for the total mass (Figure 9). This is somewhat lower than the total mass predicted by evolutionary models. Using an $H$-band bolometric correction from Casagrande et al. (2008) and the parallactic distance to the system, we find a luminosity of $\log L / L_{\odot}=-2.17 \pm 0.05$ dex for 
Table 7

Allers \& Liu 2013 Gravity Indices

\begin{tabular}{|c|c|c|c|}
\hline Parameter & 2MASS J02155892-0929121 C & G $160-54 \mathrm{C}$ & 2MASS J11240434+3808108 B \\
\hline $\mathrm{VO}_{z}$ Index & $1.023 \pm 0.011$ & $1.042 \pm 0.007$ & $1.069 \pm 0.011$ \\
\hline $\mathrm{FeH}_{z}$ Index & $1.082 \pm 0.012$ & $1.109 \pm 0.010$ & $1.210 \pm 0.015$ \\
\hline $\mathrm{K}_{\mathrm{I}_{J}}$ Index & $1.030 \pm 0.006$ & $1.072 \pm 0.005$ & $1.111 \pm 0.007$ \\
\hline$H$-cont Index & $1.005 \pm 0.006$ & $0.968 \pm 0.005$ & $0.931 \pm 0.007$ \\
\hline $\mathrm{FeH}_{J}$ Index & $1.066 \pm 0.010$ & $1.113 \pm 0.010$ & $1.202 \pm 0.019$ \\
\hline $\mathrm{EW}(\mathrm{Na}$ I $1.138 \mu \mathrm{m})(\AA)$ & $6.9 \pm 0.7$ & $12.1 \pm 0.2$ & $\cdots$ \\
\hline $\mathrm{EW}\left(\mathrm{K}_{\mathrm{I}} 1.169 \mu \mathrm{m}\right)(\AA)$ & $2.7 \pm 0.5$ & $4.3 \pm 0.4$ & $5.5 \pm 0.5$ \\
\hline $\operatorname{EW}\left(\mathrm{K}_{\mathrm{I}} 1.177 \mu \mathrm{m}\right)(\AA)$ & $4.0 \pm 0.5$ & $6.6 \pm 0.4$ & $8.8 \pm 0.5$ \\
\hline $\operatorname{EW}\left(\mathrm{K}_{\mathrm{I}} 1.253 \mu \mathrm{m}\right)(\AA)$ & $2.4 \pm 0.4$ & $4.5 \pm 0.4$ & $5.4 \pm 0.4$ \\
\hline Gravity Score ${ }^{a}$ & $\ln 12$ & On00 & On00 \\
\hline Gravity Class & INT-G & FLD-G & FLD-G \\
\hline
\end{tabular}

Note.

${ }^{a}$ Gravity scores are for the FeH, VO, alkali lines, and $H$-band continuum shape. See Allers \& Liu (2013) for details.
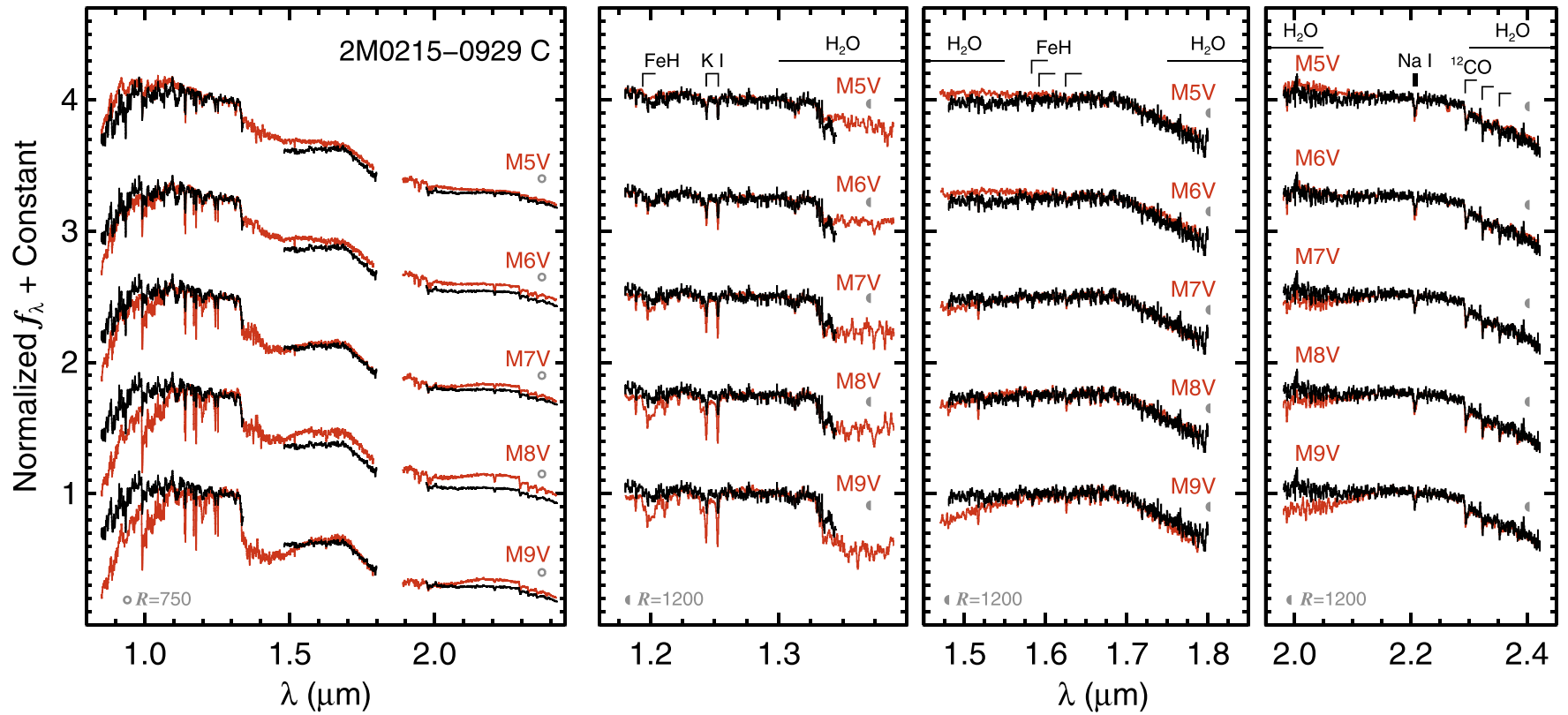

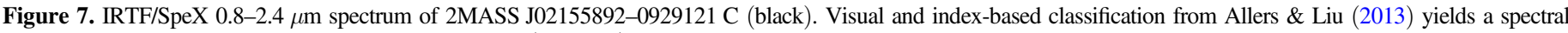

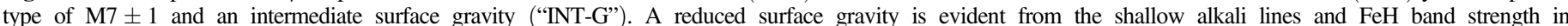

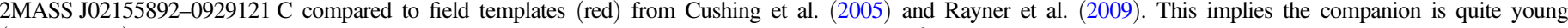

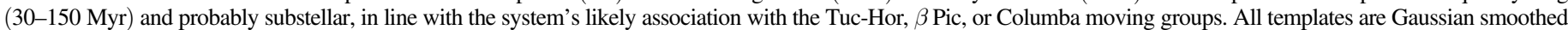

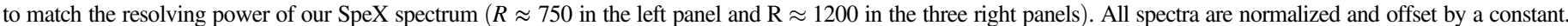

Table 8

1RXS J034231.8+121622 AB Astrometry

\begin{tabular}{lccc}
\hline \hline Epoch & $\begin{array}{c}\text { Separation } \\
(\text { mas })\end{array}$ & $\begin{array}{c}\text { PA } \\
\left({ }^{\circ}\right)\end{array}$ & Ref \\
\hline 2007.95 & $883.0 \pm 0.2$ & $17.58 \pm 0.09$ & TW \\
2008.63 & $860 \pm 8$ & $17.3 \pm 0.4$ & J12 \\
2008.87 & $866 \pm 8$ & $17.8 \pm 0.4$ & J12 \\
2010.66 & $851 \pm 3$ & $18.7 \pm 0.1$ & TW \\
2012.02 & $834 \pm 57$ & $17.6 \pm 1.7$ & J14 \\
2012.65 & $831 \pm 2$ & $18.71 \pm 0.07$ & B15 \\
2013.04 & $822 \pm 8$ & $19.1 \pm 0.7$ & B15 \\
\hline
\end{tabular}

References. B15-Bowler et al. (2015), J12-Janson et al. (2012), J14Janson et al. (2014), TW-this work.

the primary star. This corresponds to masses of $0.15,0.20,0.23$, and 0.25 for ages of $60,100,200$, and $300 \mathrm{Myr}$ using the evolutionary models of Baraffe et al. (1998). The only way the total mass is consistent with these models is for young ages ( $\lesssim 100 \mathrm{Myr}$ ), although it is also possible that systematic errors exist in the evolutionary models (Dupuy et al. 2014). Unfortunately, the relatively uncertain age of this system makes it difficult to use as a robust test of models.

\section{5. $H D 23514$ AB (2MASS J03463839+2255112)}

HD 23514 is an F6 member of the Pleiades with an unusually high fractional infrared luminosity of $\approx 2 \%$, indicating the presence of a large amount of collisionally ground dust within $\sim 1$ AU (Rhee et al. 2008). Rodriguez et al. (2012) discovered a substellar companion to HD 23514 located at 2.6" ( $\approx 360 \mathrm{AU})$ with AO imaging. HD $23514 \mathrm{~B}$ was also independently found by Yamamoto et al. (2013) as part of the Subaru SEEDS exoplanet imaging program. As a companion to a wellestablished member of the Pleiades cluster, HD $23514 \mathrm{~B}$ is an 

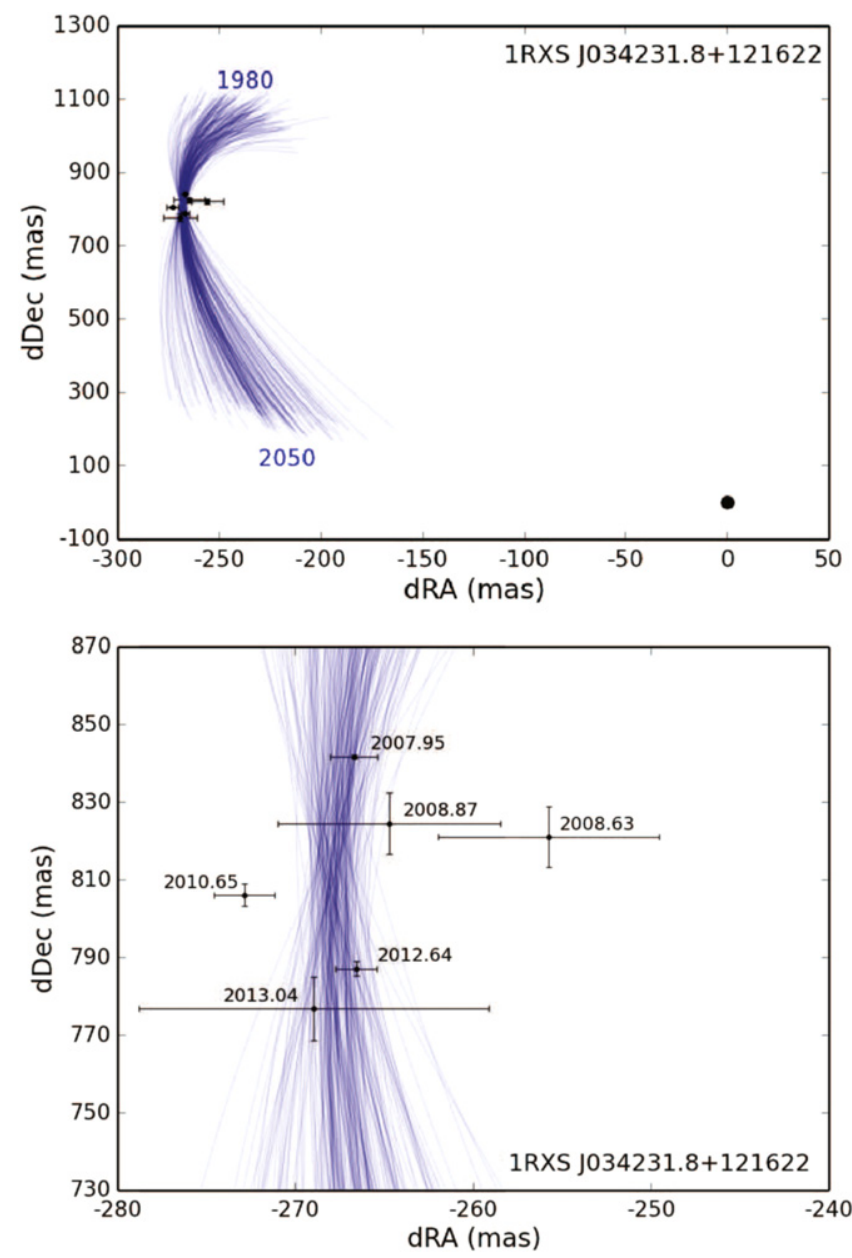

Figure 8. Orbit fits for the intermediate-age (60-300 Myr) M4+L0 binary 1RXS J034231.8+121622 AB. Although the astrometric baseline (2007-2013) spans a small fraction of the orbital period, only a subset of Keplerian orbits are consistent with the observations. Each curve represents a draw from the posterior distribution of allowed orbital elements, conditioned on the astrometric data. The orbits are plotted only from the expected position of the companion in 1980 to the expected position in 2050. In the upper panel, the black dot denotes the position of the primary star. The lower panel shows a magnified version of the upper panel.

excellent intermediate-age $(120 \pm 10 \mathrm{Myr})$ benchmark for comparative analysis with field objects of unknown ages.

Figure 10 shows our $H$ - and $K$ - band (1.5-2.4 $\mu \mathrm{m})$ spectra of HD $23514 \mathrm{~B}$ taken with Keck/OSIRIS. We have not made any correction for reddening because extinction to the Pleiades is small $(E(B-V)<0.06 \mathrm{mag}$; Taylor 2008). HD $23514 \mathrm{~B}$ exhibits typical features of late-M dwarfs including $\mathrm{FeH}$ absorption, strong $\mathrm{CO}$ bands, and $\mathrm{Na}$ I absorption. Interestingly, the $H$-band shape is only slightly angular, implying that late-M dwarfs showing even more pronounced angular morphology are younger than $120 \mathrm{Myr}$. This agrees with the conclusions by Allers \& Liu (2013), who found that intermediate and very low gravity brown dwarfs have ages $\lesssim 200$ Myr. Compared to field templates, HD 23514 B best resembles M9-L0 objects in $H$ band and M7-M8 objects in $K$ band. We therefore adopt a NIR spectral type of M8 \pm 1 . In the future, $J$-band spectroscopy of HD $23514 \mathrm{~B}$ will be useful to better calibrate the age-dependency of $J$-band alkali absorption line strengths.

\subsection{G 160-54 AabBC (2MASS J04134585-0509049 AabBC)}

G 160-54 is a little-studied system with an (unresolved) spectral type of M4.5 \pm 0.5 (Figure 1; Table 5) and weak $\mathrm{H} \alpha$ emission $(\mathrm{EW}=-0.8 \AA$ from UH $2.2 \mathrm{~m} / \mathrm{SNIFS}$ and $\mathrm{EW}=$ $-0.54 \AA$ from CFHT/ESPaDOnS). Although not detected by ROSAT, a UV counterpart appears in GALEX (FUV = $21.7 \pm 0.4 \mathrm{mag}, \mathrm{NUV}=20.79 \pm 0.17 \mathrm{mag})$ and was resolved into a close visual triple system by Bowler et al. (2015) with NIR AO imaging at Keck and Subaru. G 160-54 B is a stellar companion $0.2^{\prime \prime}(\approx 4 \mathrm{AU})$ from the primary $\mathrm{G} 160-54 \mathrm{~A}$, and $\mathrm{G} 160-54 \mathrm{C}$ is a fainter $(\Delta K=2.8 \mathrm{mag})$ comoving tertiary located at $3.3^{\prime \prime}(\approx 70 \mathrm{AU})$. No parallax or $\mathrm{RV}$ has been measured for this system, but we note that at a distance of $\approx 18.5 \mathrm{pc}$ and $\mathrm{RV}$ of $\approx 13 \mathrm{~km} \mathrm{~s}^{-1}$ its $U V W$ kinematics $\left(\{-9.9,-20.7,-0.9\} \mathrm{km} \mathrm{s}^{-1}\right)$ would precisely match known members of the Tuc-Hor moving group. At that age ( 30-40 Myr), the outer companion G 160-54 C would fall below the hydrogen-burning limit.

The very weak $\mathrm{H} \alpha$ emission, however, disagrees with established Tuc-Hor members, which without exception have line strengths larger than $-6 \AA$ at a spectral type of M4 (Kraus et al. 2014b). Cross-correlating our high-resolution spectrum of G 160-54 AB with RV standards yields three strong peaks, implying there is a fourth component in the system because $\mathrm{G} 160-54 \mathrm{C}$ is too distant and too faint in the optical to produce such a large CC peak. Since we only have a single epoch, we cannot identify whether this fourth member orbits G 160$54 \mathrm{~A}$ or $\mathrm{B}$, but for this work we assume it orbits the primary "A" component in a similar configuration as 2 MASS J02155892-0929121.

Our SpeX SXD spectrum of G160-54C is shown in Figure 11. Compared to field templates, it best resembles the M7 dwarf across the entire $0.8-2.4 \mu \mathrm{m}$ spectrum and among individual bandpasses. There are no obvious signs of low surface gravity. This is corroborated with index-based spectral and gravity classifications from Allers \& Liu (2013), which yield a NIR spectral type of $\mathrm{M} 7 \pm 0.5$ and a field gravity (FLD-G). Altogether, despite the potentially coincidental kinematic overlap with Tuc-Hor, this system is certainly older than $150 \mathrm{Myr}$ and most likely much older (>1 Gyr). At these old ages, all four components in this system are low-mass stars. Based on its distance $(21 \pm 9 \mathrm{pc})$, age $(1-10 \mathrm{Gyr})$, and $K_{S^{-}}$-band magnitude (12.24 \pm 0.06 ; Bowler et al. 2015), G 160-54 C has a mass of $85 \pm 16$ using a $K$-band bolometric correction from Liu et al. (2010a) and the Burrows et al. (1997) evolutionary models. The probability it is substellar $\left(<75 M_{\mathrm{Jup}}\right)$ is only $7 \%$.

\subsection{MASS J05464932-0757427 AB}

The M3.0 primary star 2MASS J05464932-0757427 A is not particularly noteworthy, as it was not detected by ROSAT nor GALEX. Janson et al. (2012) identified a moderate-contrast companion $\left(\Delta z^{\prime}=5.6 \mathrm{mag}\right)$ at $2.8^{\prime \prime}$ in two epochs of imaging in 2008 and 2009. We imaged the system in $K_{S}$ band with NIRC2 in 2013. The known secondary was clearly detected, and there are no signs of additional companions in the system. Janson et al. estimate a spectral type of >LO for the companion based on $z^{\prime}$ - and $i^{\prime}$-band contrasts. Our $K_{S}$-band contrast of 3.9 mag corresponds to an apparent magnitude of 13.7 mag and an absolute $K_{S}$-band magnitude of $\approx 10.4$ mag at the estimated distance of $\approx 45 \mathrm{pc}$ from Janson et al. Assuming a $20 \%$ uncertainty in distance, the implied mass of 2MASS 

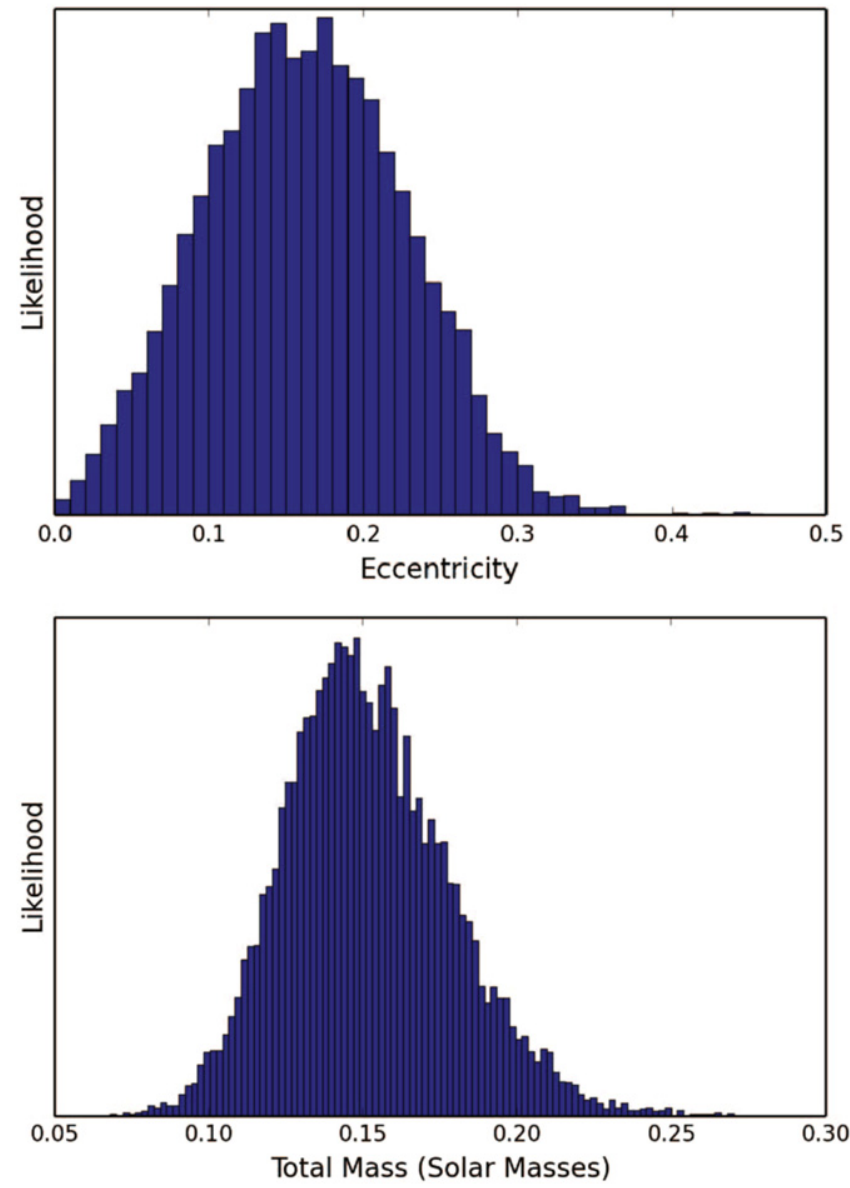

Figure 9. Posterior probability distributions for the eccentricity and total mass of 1RXS J034231.8+121622 AB (see Figure 8). Initial orbit fits of this system point to a low eccentricity $(\lesssim 0.3)$ and a total mass of $\approx 0.1-0.2 M_{\odot}$.
J05464932-0757427 B is $87 \pm 3 M_{\text {Jup }}$ for an age range of 1-10 Gyr using a bolometric correction from Liu et al. (2010a) and evolutionary models from Burrows et al. (1997).

\subsection{MASS J06475229-2523304 AabB}

2MASS J06475229-2523304 is an active late-K-type star first recognized by Riaz et al. (2006), who found weak $\mathrm{H} \alpha$ emission $(\mathrm{EW}=-1.1 \AA$ ) and a saturated X-ray fractional luminosity $\left(\log L_{X} / L_{\mathrm{bol}}=-3.13 \mathrm{dex}\right)$. There are various conflicting classifications in the literature. Riaz et al. (2006) find a spectral type of $\mathrm{K} 7 \mathrm{~V}$ from low-resolution optical spectroscopy, Torres et al. (2006) find K1IIIJe from highresolution optical spectroscopy, and Pickles \& Depagne (2010) infer K3III from photometric fits to synthetic spectra spanning optical to NIR wavelengths. To better characterize this star we obtained a UH $2.2 \mathrm{~m} / \mathrm{SNIFS}$ low-resolution optical spectrum spanning $\approx 3500-8500 \AA$. Figure 12 compares our UH $2.2 \mathrm{~m} /$ SNIFS spectrum to late-type templates from Bochanski et al. (2007) and Mann et al. (2013). 2MASS J06475229-2523304 broadly resembles K7/M0 dwarfs, and we find a consistent index-based spectral type of K7 using Hammer (Covey et al. 2007). We therefore adopt a K7.0 \pm 0.5 classification for 2MASS J06475229-2523304.

The band depths in our UH $2.2 \mathrm{~m} / \mathrm{SNIFS}$ spectrum do not match dwarf templates in Figure 12, however, which is confirmed via spectral indices from Reid et al. (1995). We measure values of $0.890,0.950$, and 0.952 for the TiO5, $\mathrm{CaH} 2$, and $\mathrm{CaH} 3$ indices, which sit above the dwarf locus in $\mathrm{CaH} 2+\mathrm{CaH} 3$ versus the TiO5 diagram in a region occupied by giant stars (e.g., Mann et al. 2012). This points to a low surface gravity, indicating 2MASS J06475229-2523304 is most likely a very young or evolved (subgiant or giant) star. The NIR colors of 2MASS J06475229-2523304 $(J-H=0.59 \pm$ $0.04 \mathrm{mag}, H-K_{S}=0.21 \pm 0.04 \mathrm{mag}$ ) are similar to both late-K/
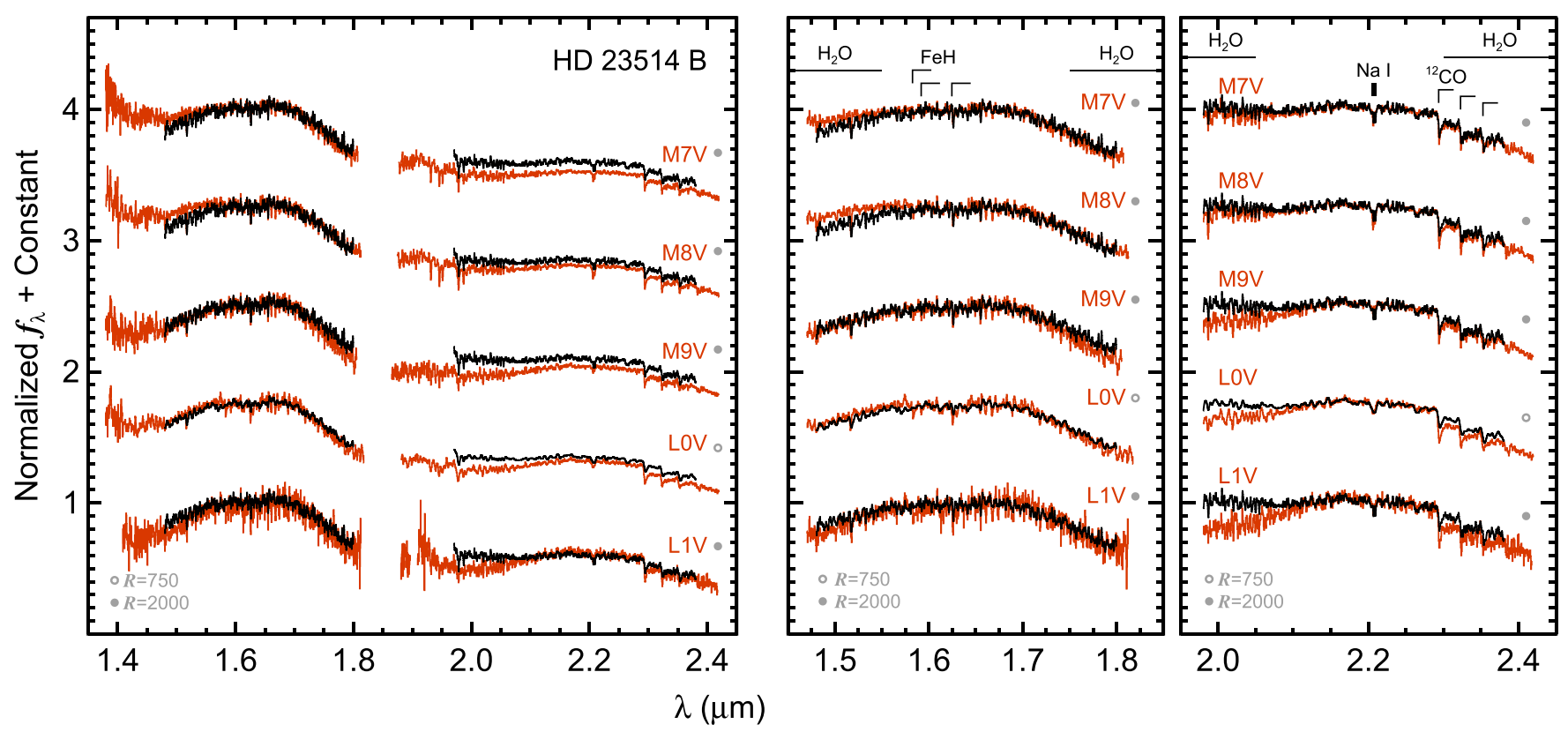

Figure 10. Keck/OSIRIS 1.4-2.4 $\mu \mathrm{m}$ spectrum of the substellar companion to the dusty F6 Pleiades star HD 23514. HD 23514 B best resembles field M7-L0 templates (red) from the IRTF Spectral Library. Our OSIRIS $H$ and $K$ spectra (black) are flux-calibrated to photometry from Rodriguez et al. (2012). All spectra are Gaussian smoothed to a common resolving power, normalized, and offset by a constant. 

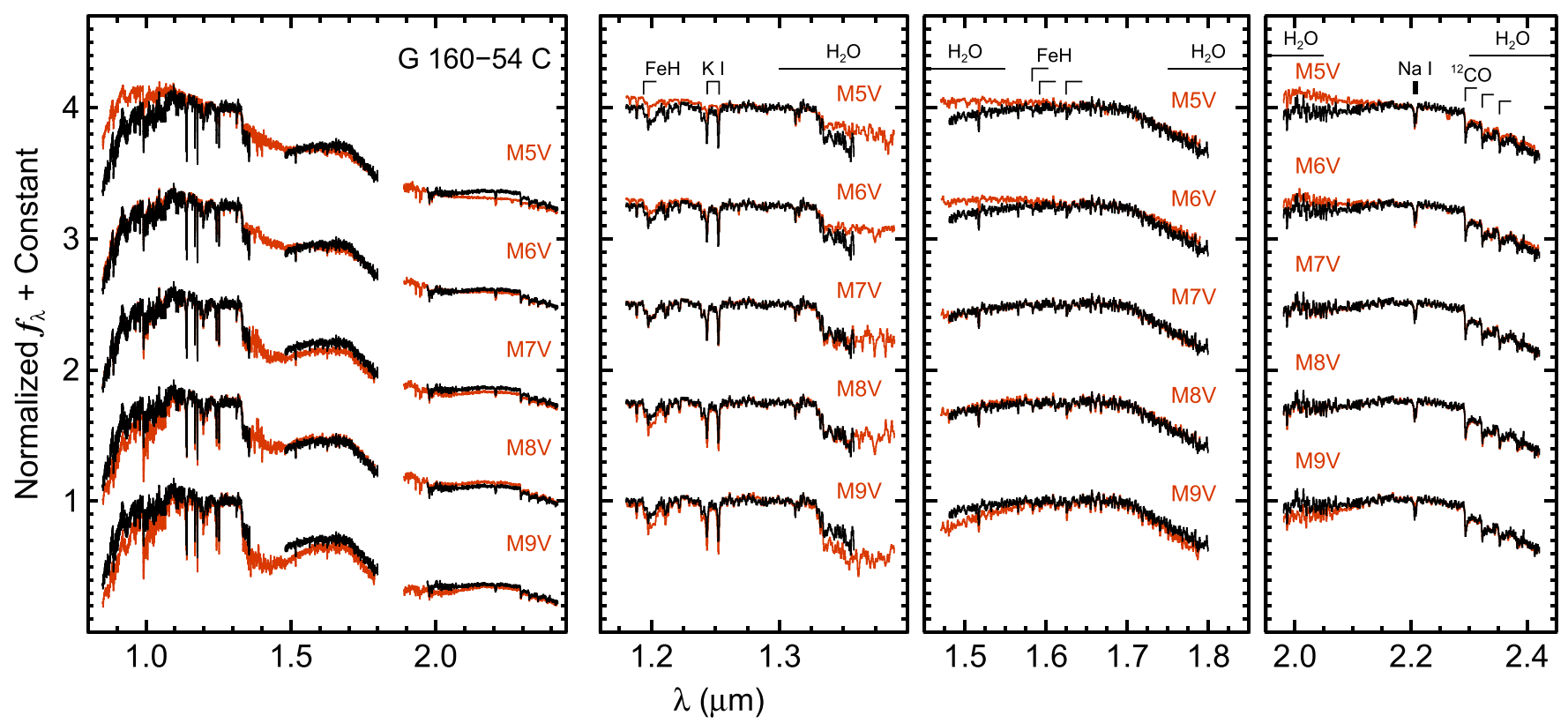

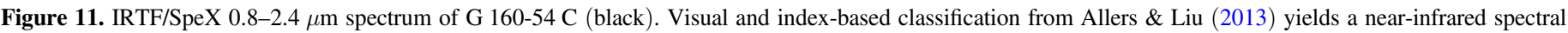

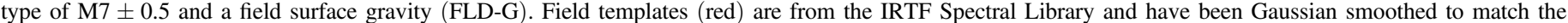
resolving power of our SpeX $\operatorname{spectrum}(R \approx 1200)$. All spectra are normalized and offset by a constant.

early-M dwarfs and giants and cannot be used to distinguish between these evolutionary stages; only at later spectral types do their branches diverge in NIR color-color diagrams (e.g., Bessell \& Brett 1988). The reduced proper motion of 2MASS J06475229-2523304 $\left(H_{V} \equiv V+5 \log (\mu)+5\right.$, where $\mu$ is the total proper motion in arcseconds per year) is $10.3 \mathrm{mag}$, which is more consistent with field dwarf-like kinematic and photometric properties instead of distant giant stars with high luminosities and low tangential velocities (e.g., Lépine \& Gaidos 2011).

Based on its activity and proper motion, Malo et al. (2013) suggest 2MASS J06475229-2523304 is a possible member of Columba or AB Dor, but they note that neither group assignment is compatible with the measured RV of $-56.5 \mathrm{~km} \mathrm{~s}^{-1}$ from Torres et al. (2006). Malo et al. (2014) find an RV of $-24.4 \pm 0.8 \mathrm{~km} \mathrm{~s}^{-1}$ measured in 2011 , and the RAVE survey (Kordopatis 2013) finds an RV of $-67 \pm 6 \mathrm{~km} \mathrm{~s}^{-1}$ measured in 2009, suggesting the primary is an SB1. Indeed, Torres et al. also listed this target as a possible binary ("SB1?"). We confirm this with our own RVs of $-2.1 \pm 0.9 \mathrm{~km} \mathrm{~s}^{-1}$ and $-5.5 \pm 0.8 \mathrm{~km} \mathrm{~s}^{-1}$ obtained in 2013 and 2014. Our du Pont spectrum from 2013 shows slight $\mathrm{H} \alpha$ emission $(\mathrm{EW}=-0.5 \AA)$ with an asymmetric line profile; however, there are no signs of a companion in the crosscorrelation function. Hereinafter this $\mathrm{SB}$ is referred to as 2MASS J06475229-2523304 Aab.

There is not yet a parallax measurement for this system. Riaz et al. (2006) find a $J$-band spectrophotometric distance of $45 \pm 17 \mathrm{pc}$, and Ammons et al. (2006) estimate a distance of $94_{-35}^{+88}$ pc from SED fits. Assuming a Pleiades-like age, we infer an absolute $V$-band magnitude of $8.11 \pm 0.09 \mathrm{mag}$ and a photometric distance of $36 \pm 2$ pc using the relation from Bowler et al. (2013). If 2MASS J06475229-2523304 Aab is younger than $\sim 120 \mathrm{Myr}$, or if it is an evolved star, then its distance will be farther. Although we do not have a systemic $\mathrm{RV}$, we note that no combination of $\mathrm{RV}$ between -50 and
$-2 \mathrm{~km} \mathrm{~s}^{-1}$ and distance between $10-100 \mathrm{pc}$ is consistent with the $X Y Z$ spatial positions of known moving groups.

\subsubsection{The Comoving Companion 2MASS J06475229-2523304 B}

In 2012 we resolved a close $\left(1.1^{\prime \prime}\right)$, modest-contrast $(\Delta K=$ $5.5 \mathrm{mag}$ ) point source near 2MASS J06475229-2523304 with Subaru/IRCS AO imaging (Figure 13). Follow-up astrometry with Keck/NIRC2 in 2013 and 2014 confirms the candidate is comoving with the primary star (Figure 14). No orbital motion is detected.

Torres et al. (2006) cite $60 \mathrm{m \AA} \mathrm{Li}$ I absorption from their high-resolution spectrum of the primary star. Regardless of its kinematics, this would imply an age range of $\sim 40-150 \mathrm{Myr}$, assuming the system is indeed young rather than evolved, which raises the possibility that 2MASS J06475229$2523304 \mathrm{~B}$ is a new young brown dwarf companion. However, we do not detect any $\mathrm{Li}$ I absorption with our own highresolution du Pont/Echelle and SMARTS $1.5 \mathrm{~m} / \mathrm{CHIRON}$ spectra, pointing to an age $\gtrsim 100 \mathrm{Myr}$ and a companion mass of about 40,85 , and $100 M_{\text {Jup }}$ for distances of 40,70 , and $100 \mathrm{pc}$, respectively.

In 2014 December we obtained a $K$-band spectrum of 2MASS J06475229-2523304 B with Keck/OSIRIS NGSAO. The spectrum does not resemble a late-M/early-L spectral type as expected for a young $(\lesssim 200 \mathrm{Myr})$, nearby (within $\sim 100 \mathrm{pc}$ ) system. Instead, the best match is an M3 template with no obvious signs of low surface gravity (Figure 15). This has forced us to reevaluate the nature of this triple system because the component spectral types of K7 and M3 conflict with our measured NIR flux ratio of $5.5 \mathrm{mag}$. The corresponding effective temperatures of K7 and M3 dwarfs are 3960 and $3412 \mathrm{~K}$ (Boyajian et al. 2012), respectively, which translates into a $K$-band contrast ratio of $\approx 1.7-1.9 \mathrm{mag}$, regardless of the system age (Baraffe et al. 1998). In other words, 2MASS J06475229-2523304 B is at least 3.5 mag fainter than 


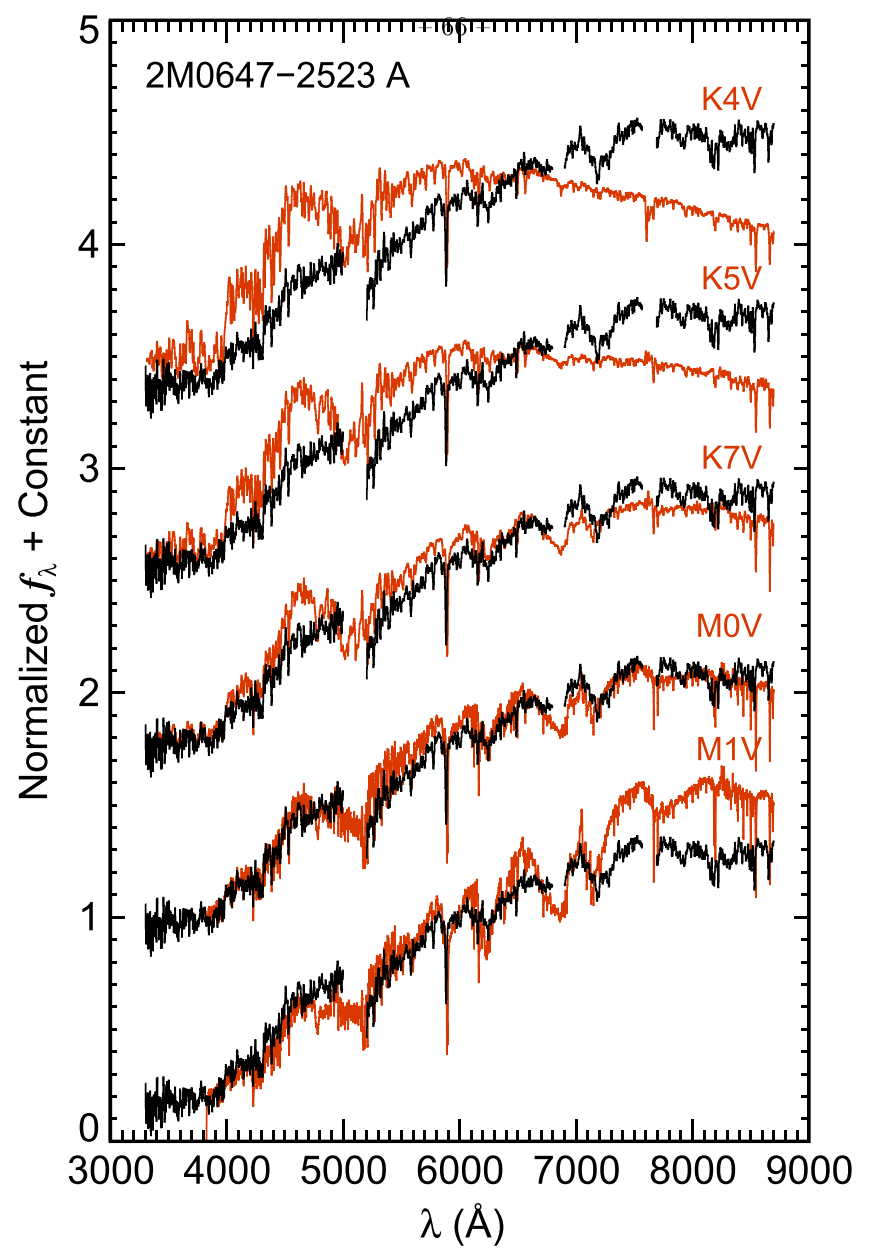

Figure 12. UH $2.2 \mathrm{~m} / \mathrm{SNIFS}$ spectrum of 2MASS J06475229-2523304 A (black) compared to field K- and M-dwarf templates (red) from Mann et al. (2013) and Bochanski et al. (2007), respectively. 2MASS J064752292523304 A broadly resembles the K7V/M0V templates, but the detailed molecular band strengths are not a good match. Instead, index measurements point to a low surface gravity. The $\mathrm{H} \alpha$ line is slightly in emission $(\mathrm{EW}=-0.3 \AA$ ). Spectral regions with strong telluric absorption have been removed, and all spectra are normalized and offset by a constant.

it should be, assuming all stars in the system are on the main or pre-main sequence.

\subsubsection{A Subgiant and M-Dwarf Binary?}

Since it appears unlikely that 2MASS J06475229-2523304 AabB is composed of normal stars on the main or pre-main sequence, here we discuss a few alternative explanations. One option is that the system is very young and an edge-on protoplanetary disk surrounding 2MASS J06475229-2523304 B is producing $\sim 3.5-4 \mathrm{mag}$ of extinction in the NIR, similar to TWA 30 B (Looper et al. 2010) and possibly FW Tau (Bowler et al. 2014; Kraus et al. 2014a). However, this would require the system to be younger than the disk dissipation timescale of $\sim 10 \mathrm{Myr}$, in which case we would expect lithium absorption in the primary to be similar to TWA members (at least $300 \mathrm{~m} \AA$; e.g., Mentuch et al. 2008). This age is definitively ruled out with the nondetection of $\mathrm{Li}$ I in our high-resolution optical spectrum, so this edge-on disk scenario is unlikely.

Another option is that the primary is an evolved star while the companion is on the main sequence. Using the $J$-band
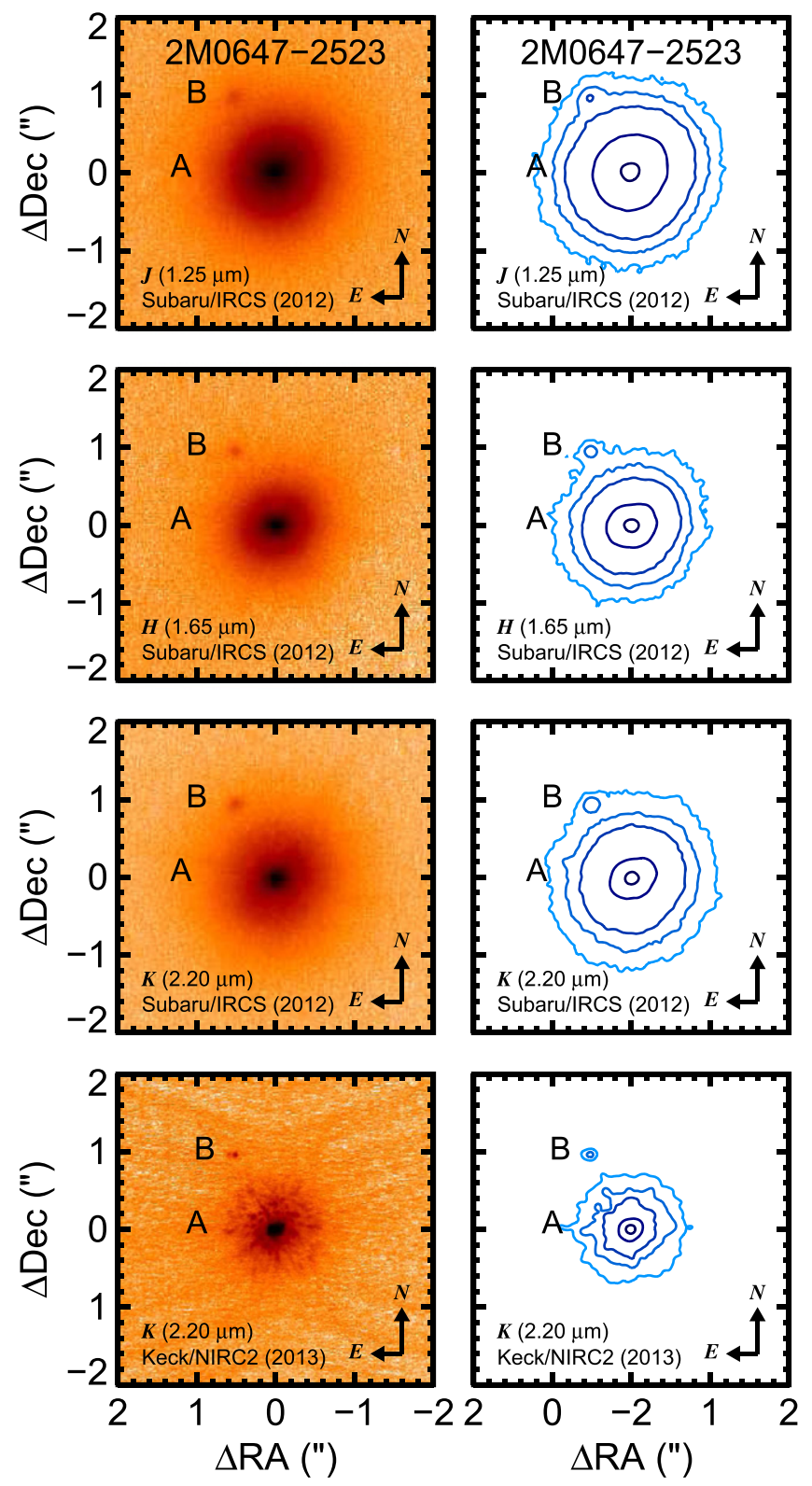

Figure 13. Subaru/IRCS and Keck/NIRC2 AO images of 2MASS J064752292523304 AB between 2012 and 2013. The companion is easily resolved, with a separation of $1.1^{\prime \prime}$ at both epochs. Contours represent $0.02 \%, 0.5 \%, 1 \%, 5 \%$, and $50 \%$ of the peak flux after convolution with a Gaussian kernel with a FWHM equal to that of the image PSF. North is up, and east is left. The field of view is $4^{\prime \prime} \times 4^{\prime \prime}$.

contrast, the typical $V-J$ color of M3 dwarfs from Pecaut \& Mamajek (2013), and the absolute $V$-band magnitude of M3 dwarfs from Drilling \& Landolt (2000), we find a spectrophotometric distance of $240 \pm 40 \mathrm{pc}$ to 2MASS J06475229$2523304 \mathrm{~B}$. If we assume the primary is a red giant, its apparent $V$-band magnitude implies a distance of $1700 \pm 100 \mathrm{pc}$. Instead, to match the distance estimate of $240 \mathrm{pc}$ for the companion, 2MASS J06475229-2523304 A would need to have an absolute $V$-band magnitude of $\sim 4.0 \mathrm{mag}$. This is above the main sequence even if the primary is an equal-flux binary and is most consistent with evolved stars on the subgiant branch. Pojmanski (1997) and Richards et al. (2012) measure a rotation rate of 7.56 days for 2MASS J06475229-2523304 A, which is somewhat faster than the typical rotation period for 


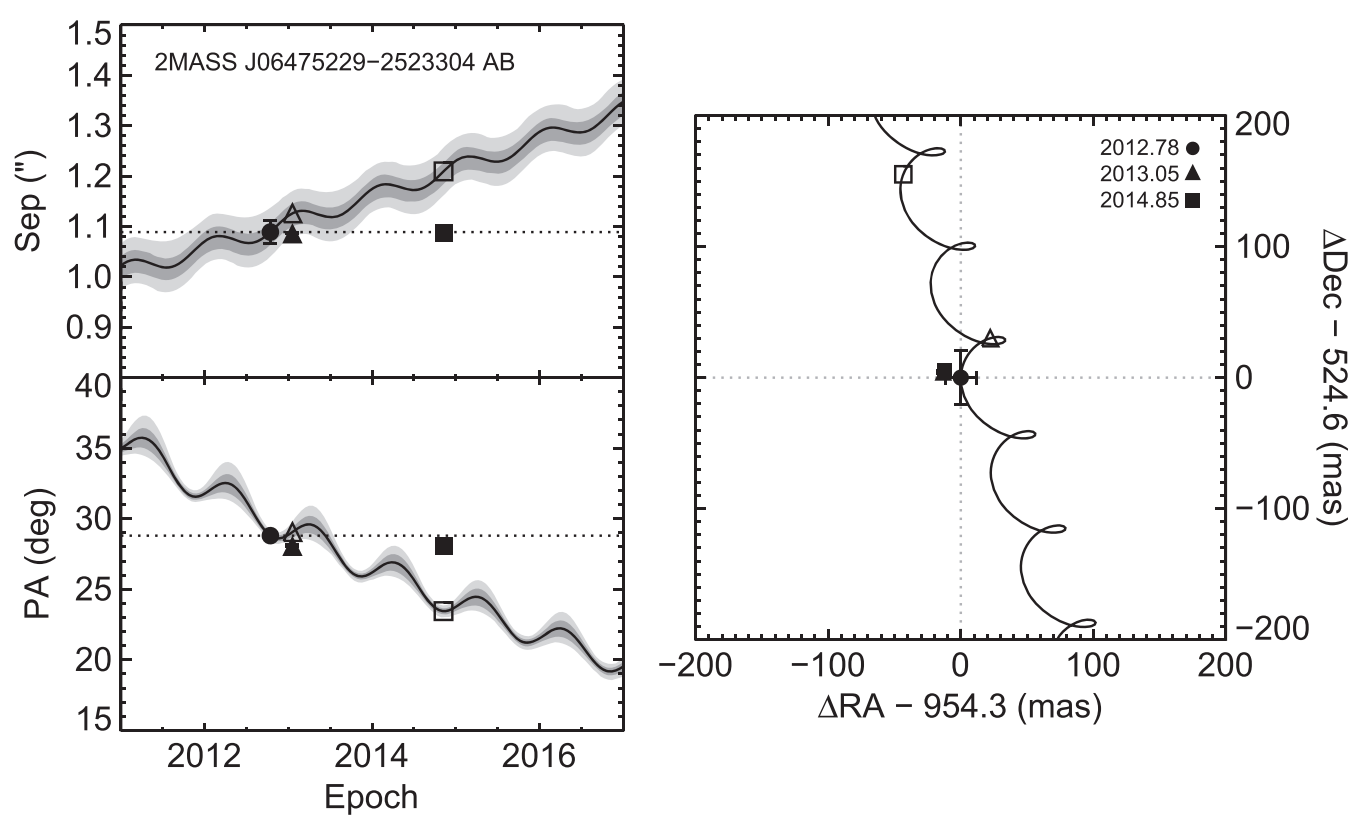

Figure 14. Relative astrometry of 2MASS J06475229-2523304 AB between 2012 and 2014 (filled symbols). The solid curve shows the expected path of a background object, and gray shaded regions show $1 \sigma$ and $2 \sigma$ confidence intervals. Open symbols represent the predicted astrometry for a stationary object at the epochs of the observations. The pair are clearly comoving and physically bound.

subgiants of $\sim 10-100$ days (do Nascimento et al. 2012; van Saders \& Pinsonneault 2013). However, the fast rotation period can be explained by a tidally locked short-period companion (e.g., Ryan \& Deliyannis 1995), which can also account for its $\mathrm{RV}$ variations, $\mathrm{H} \alpha$ emission, and X-ray activity. We conclude that this system is most likely a distant $(\sim 240 \mathrm{pc})$ triple composed of an active $\mathrm{K} 7$ subgiant primary with a close, likely tidally locked companion and a much wider ( 260 AU) M3 dwarf.

\subsection{MASS J08540240-3051366 AB}

This nearby active M4.0 star was detected in ROSAT and GALEX and shows fairly strong $\mathrm{H} \alpha$ emission (Riaz et al. 2006, $\mathrm{EW}=-8.4 \AA$ ). Its $G A L E X$ photometry $(\mathrm{FUV}=21.1 \pm 0.4$ mag, NUV $=19.53 \pm 0.15)$ points to activity levels comparable to known moving group members in UV/NIR color-color diagrams (e.g., Bowler et al. 2012b). Chauvin et al. (2010) estimate an age of $100 \mathrm{Myr}$ based on its saturated X-ray luminosity $\left(\log L_{X} / L_{\mathrm{bol}}=-2.79 \mathrm{dex}\right.$; Riaz et al. 2006). With no $\mathrm{RV}$, the BANYAN tool for calculating moving group membership probabilities (Malo et al. 2013; Gagné et al. 2014) predicts a high a priori value (87\%) that 2MASS J08540240-3051366 is a member of $\beta$ Pic. We measure an RV of $44.5 \pm 0.6 \mathrm{~km} \mathrm{~s}^{-1}$ from our high-resolution optical spectrum obtained in 2009. Based on this value, 2MASS J08540240-3051366 does not appear to be a member of a known moving group.

Chauvin et al. (2010) first imaged this star as part of their AO imaging search for planets around young southern stars with VLT/NaCo. They identified a modest-contrast $(\Delta K$ $=2.8 \mathrm{mag}$ ) companion located at $1.7^{\prime \prime}$. Assuming an age less than 200 Myr and a distance of $\approx 10-15 \mathrm{pc}$ as inferred by Riaz et al. and Chauvin et al., the mass of 2MASS J08540240-3051366 B is below $\approx 50 M_{\text {Jup }}$. Janson et al. (2012) and Janson et al. (2014) present additional Lucky imaging of the system between 2010 and 2012 and find hints of orbital motion, which we confirm with our own NIRC2 images in 2012 .
In 2014 December we obtained Keck/OSIRIS $H$ - and $K$ band spectroscopy of 2MASS J08540240-3051366 B. Compared to field templates in Figure 16, it best resembles M4-M6 spectral types with no significant deviations from field objects that would point to an especially low surface gravity. It is therefore inconsistent with $\beta$ Pic membership. We adopt a NIR spectral type of $\mathrm{M} 5 \pm 0.5$, which is slightly earlier than the classification of $\sim \mathrm{M} 7 \pm 2$ suggested by our photometry in Table 3.

Assuming an age of 100-200 Myr and a distance of $13 \pm 5 \mathrm{pc}$, the implied mass of 2MASS J08540240- 3051366 B is $42 \pm$ $21 M_{\text {Jup }}$ based on the $K$-band bolometric correction from Liu et al. (2010a) and evolutionary models from Burrows et al. (1997). On the other hand, if the system is older, then its mass is closer to the hydrogen-burning limit (e.g., $78 \pm 19 M_{\text {Jup }}$ at 0.5-1 Gyr).

\subsection{MASS J11240434+3808108 AB}

This M4.5 star is a likely member of the $\sim 500 \pm 100 \mathrm{Myr}$ Ursa Major moving group (see Bowler et al. 2015 for a detailed summary). Close et al. (2003) and Cruz et al. (2003) first noticed the late-M companion 2MASS J11240434+3808108 B located at $8.3^{\prime \prime}(\approx 170 \mathrm{AU})$ from the primary star. No additional companions were identified in deep AO imaging (Bowler et al. 2015). We obtained a moderate-resolution spectrum of 2MASS J11240434+3808108 B with IRTF/SpeX (Figure 17) and optical AO imaging with Robo-AO (Figure 18). We find an NIR spectral type of M9.5 \pm 0.5 using the spectral indices from Allers \& Liu (2013) and visual comparison to field templates, which is somewhat later than the optical type of M8.5 found by Cruz et al. (2003). The gravity indices of Allers \& Liu 2013 return a field surface gravity (FLD-G) with no signs of youth. This independently constrains the age of the system to $\gtrsim 100 \mathrm{Myr}$, consistent with the tentative association to the Ursa Major moving group. 


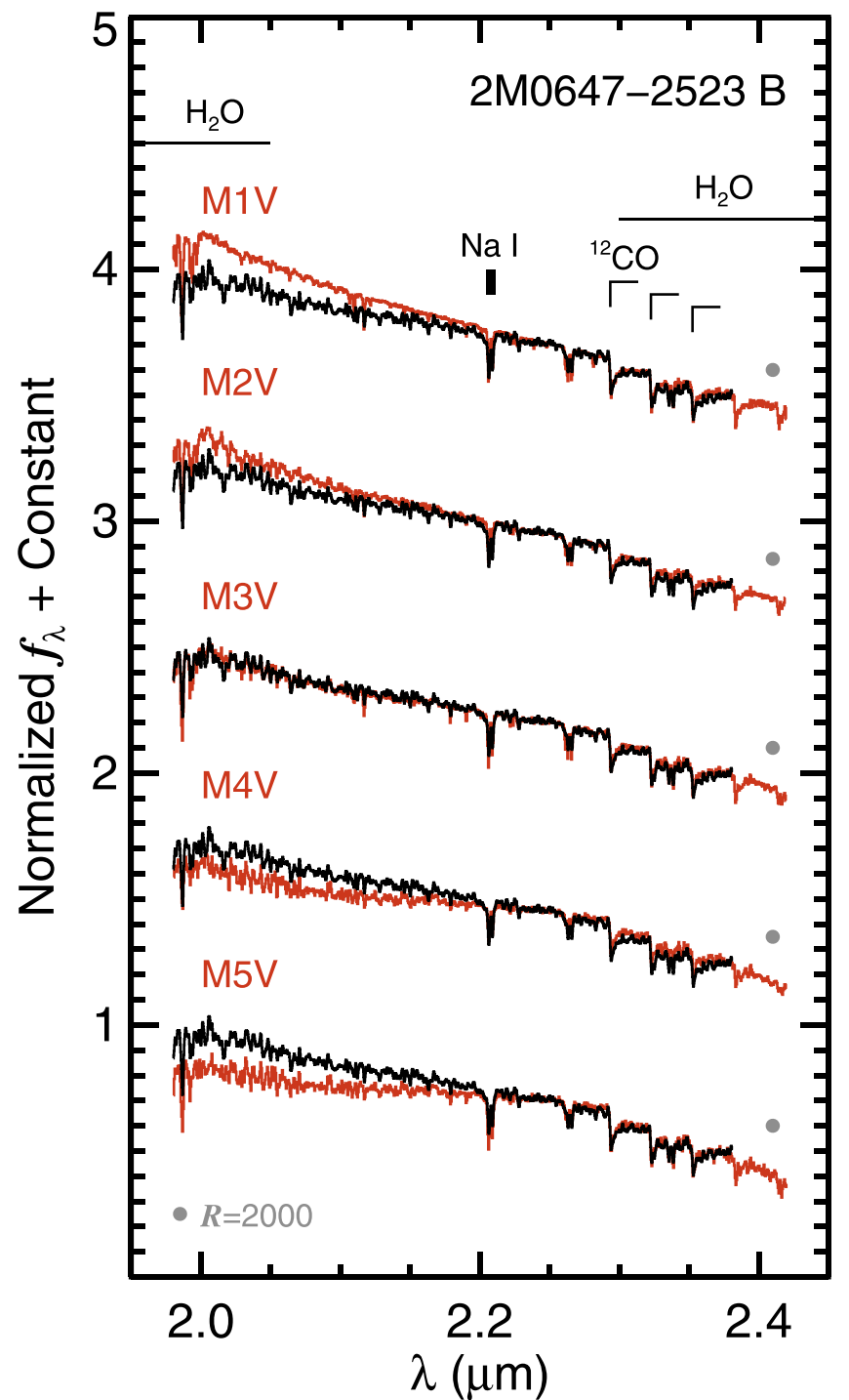

Figure 15. Keck/OSIRIS $K$-band spectrum of 2MASS J06475229-2523304 B (black). Despite being $5.5 \mathrm{mag}$ fainter than the K7 host star 2MASS J06475229-2523304 A in the NIR, this companion has a spectral type of M3V based on comparison to the field template (red) from Rayner et al. (2009). This suggests one or both components are not on the main sequence; the most likely scenario is the system is a distant $(240 \mathrm{pc}) \mathrm{K} 7$ subgiant with an M3 companion. Our OSIRIS spectrum has been Gaussian smoothed to match the resolving power of the templates $(R \approx 2000)$, and all spectra are normalized and offset by a constant.

\subsection{PYC J11519+0731 AabB (2MASS J11515681+0731262 AabB)}

\subsubsection{Spectral Classification and Binarity of PYC J11519+0731 Aab}

PYC J11519+0731 has received little previous attention in the literature aside from identification as a ROSAT source counterpart (Zickgraf et al. 2003; Haakonsen \& Rutledge 2009) and measurement of a 2.391 day rotationally modulated photometric period (Kiraga 2012). It was identified as a candidate member of the $\beta$ Pic moving group by Schlieder et al. (2012b) from its proper motion and strong activity traced by ROSAT X-ray and GALEX UV emission.

Schlieder et al. (2012a) and Lépine et al. (2013) classify PYC J11519+0731 as M3 and M2.5, respectively, from moderate-resolution optical spectroscopy. Our Mayall/RC-
Spectrograph optical spectrum and IRTF/SpeX NIR spectrum are shown in Figures 19 and 20. Even at the relatively low resolving power of our Mayall spectrum $(R \sim 2500)$, the $\mathrm{H} \alpha$ emission line $(\mathrm{EW}=-2.8 \AA)$ is marginally resolved into two components (inset). This is easily split into a double-lined spectroscopic binary (SB2) at higher resolutions (see below). Visual comparison to M-dwarf templates from Bochanski et al. (2007) indicates an M2 optical spectral type. Our spectrum deviates somewhat from the templates below $\approx 7100 \AA$, which is probably caused by slit loss from differential chromatic refraction and/or the unresolved binarity. Altogether we adopt a spectral type of M2.0 \pm 0.5 . Compared to the NIR spectrum of the M2.0 V star Gl 806 from Rayner et al. (2009) in Figure 20, PYC J11519+0731 shows some peculiarities in the form of deeper molecular and atomic absorption features primarily between $1.50-1.65 \mu \mathrm{m}$, which could reflect its SB2 nature or a supersolar metallicity. Indeed, Schlieder et al. found rather large Na I $\lambda 8200$ absorption $(\mathrm{EW}=4.3 \AA$ ), which is above the median value for field-age dwarfs of this type (see Figure 3 in Schlieder et al. 2012a) and points to a high surface gravity and old age.

We obtained three epochs of high-resolution optical spectroscopy of PYC J11519+0731 with Keck/HIRES, ESO-MPG $2.2 \mathrm{~m} / \mathrm{FEROS}$, and CFHT/ESPaDOnS between 2012 and 2014 (Table 4). Visual inspection of each spectrum reveals prominent emission and absorption features split into doublets (Figure 21), clearly indicating the primary star (PYC J11519 $+0731 \mathrm{~A}$ ) is itself a double-lined SB caught with the components at a relatively large velocity (close physical) separation. Our HIRES spectrum exhibits strong, doublepeaked $\mathrm{H} \alpha$ emission with dual self-absorption. The FEROS spectrum shows double-peaked $\mathrm{Ca}$ II $\mathrm{H}$ and $\mathrm{K}$ emission, strong $\mathrm{H} \alpha$ emission with dual self-absorption, dual reversed-emission cores in each of the $\mathrm{Ca}$ II IR triplet and the $\mathrm{NaD}$ lines, He I $\lambda 5876$ emission, and single-peaked $\mathrm{H} \beta, \mathrm{H} \gamma$, and $\mathrm{H} \delta$ emission. Similarly, the ESPaDOnS spectrum displays doublepeaked $\mathrm{Ca}$ II $\mathrm{H}$ and $\mathrm{K}$ emission, strong $\mathrm{H} \alpha$ emission with dual self-absorption, dual reversed-emission cores in each of the $\mathrm{Ca}$ II IR triplet and the $\mathrm{NaD}$ lines, dual $\mathrm{He}_{\text {I }} \lambda 5876$ emission, and dual-peaked $\mathrm{H} \beta, \mathrm{H} \gamma, \mathrm{H} \delta$, and $\mathrm{H} \epsilon$ emission.

We used a CC package from Bender \& Simon (2008) to determine the RV of the primary (Aa) and secondary $(\mathrm{Ab})$ at each epoch, systemic velocity, and mass ratio of the system. Following the methods of Mazeh et al. (2002), we performed $\mathrm{CCs}$ between the science spectrum and template spectra to measure the RV of each component. The templates can be observed standards or models, and the routine automatically accounts for the relative normalization of the spectra.

The HIRES, FEROS, and ESPaDOnS spectra were analyzed in a similar fashion. The echelle orders were first trimmed to avoid telluric features, normalized, and, when necessary, the continua were flattened by fitting and dividing by a polynomial. A set of K5-M4 RV templates were selected from Prato et al. (2002) and observed using FEROS. These same templates were used for the HIRES and ESPaDOnS analysis. We attempted $\mathrm{CC}$ with single, binary, and rotationally broadened templates. For our FEROS data, the strongest CC power $(\sim 90 \%)$ was achieved for the primary and secondary using an $\mathrm{M} 2.5+\mathrm{M} 2.5$ system with $R V_{P}=15.7 \pm 1 \mathrm{~km} \mathrm{~s}^{-1}$ and $v_{P} \operatorname{sini}_{P}=14 \pm 2 \mathrm{~km} \mathrm{~s}^{-1}$ and $R V_{S}=-18.2 \pm 1 \mathrm{~km} \mathrm{~s}^{-1}$ and $v_{S} \sin i_{S}=12 \pm 2 \mathrm{~km} \mathrm{~s}^{-1}$. The RV uncertainties are dominated by the use of observed template spectra, and the projected 

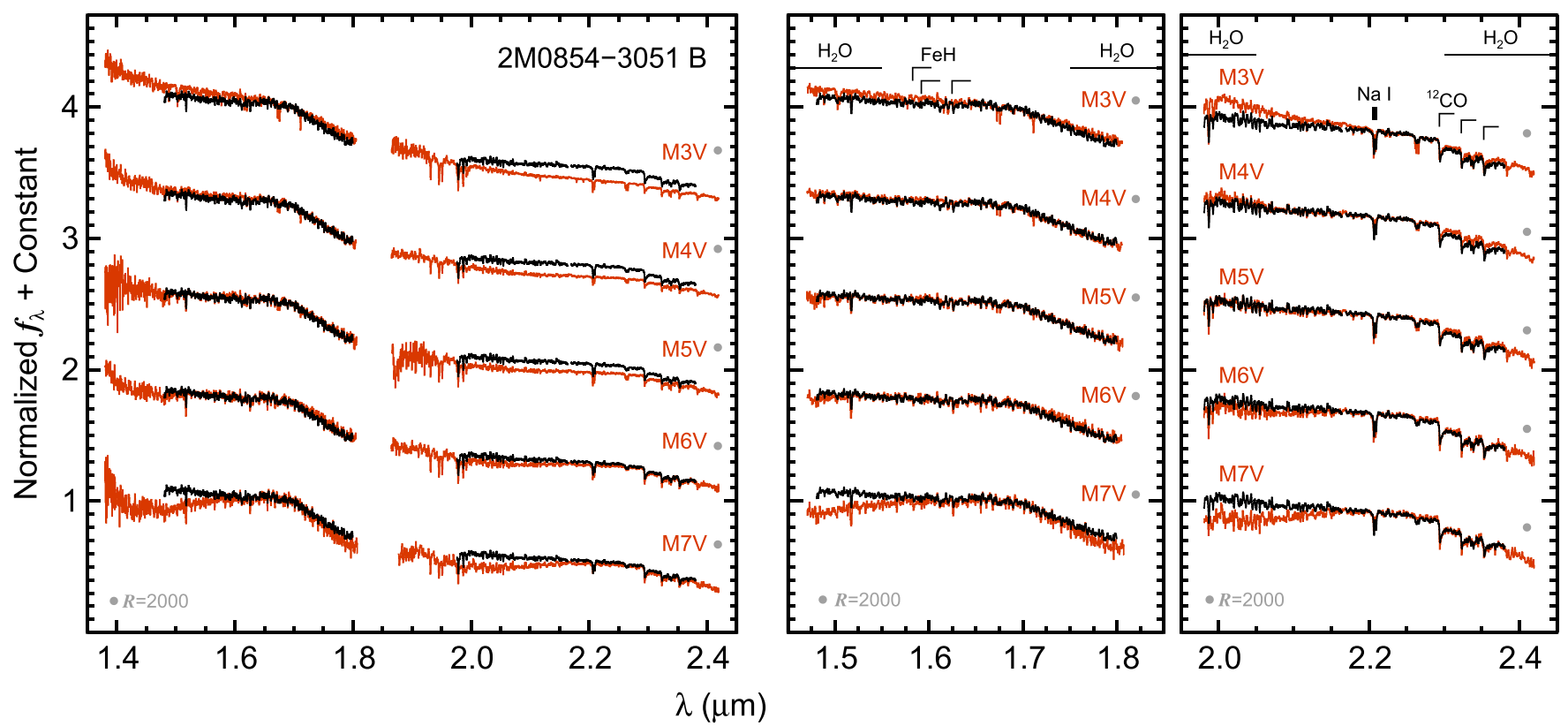

Figure 16. Keck/OSIRIS 1.5-2.4 $\mu \mathrm{m}$ spectrum of 2MASS J08540240-3051366 B (black). The best match is to M4-M6 field templates (red) from the IRTF Spectral Library; we adopt a near-infrared spectral type of M5 \pm 0.5 . Our OSIRIS spectrum has been Gaussian-smoothed to match the resolving power of the templates $(R \approx$ 2000), and all spectra are normalized and offset by a constant.
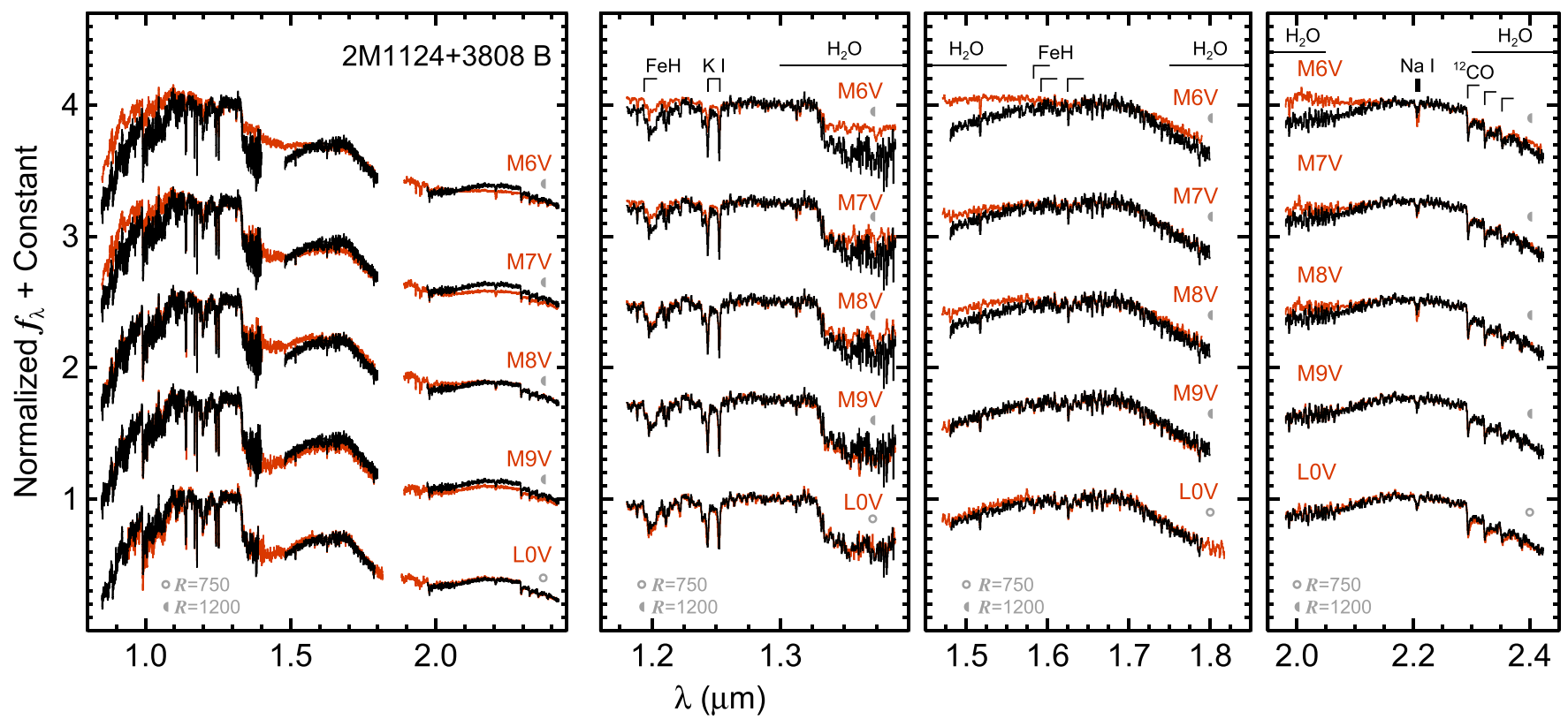

Figure 17. IRTF/SpeX 0.8-2.4 $\mu$ m spectrum of 2MASS J11240434+3808108 B (black). Visual and index-based classification from Allers \& Liu (2013) yields a near-infrared spectral type of M9.5 \pm 0.5 and a field surface gravity (FLD-G). Field templates (red) are from the IRTF Spectral Library, and the M6V-M9V objects have been Gaussian-smoothed to match the resolving power of our SpeX spectrum $(R \approx 1200)$. The L0 template has $R \approx 750$, so our science spectrum has been smoothed to match that resolution. All spectra are normalized and offset by a constant.

rotational velocity uncertainties reflect the velocity increments used to generate the suite of broadened templates. For our HIRES data we measure CC peaks at $R V_{P}=50.9 \pm 1 \mathrm{~km} \mathrm{~s}^{-1}$ and $R V_{S}=-51.9 \pm 1 \mathrm{~km} \mathrm{~s}^{-1}$, and for our ESPaDOnS data we find $R V_{P}=69.5 \pm 1 \mathrm{~km} \mathrm{~s}^{-1}$ and $R V_{S}=-75.5 \pm 1 \mathrm{~km} \mathrm{~s}^{-1}$. The range of flux ratios we infer are between $0.5-0.75$ at $\approx 6400 \AA$.

With three epochs of RV data for each component, the mass ratio of the system $(q)$ and systemic $\operatorname{RV}(\gamma)$ can be determined following the methods of Wilson (1941). Figure 22 shows the component velocities plotted against each other; the mass ratio of the system is the negative of the slope of the line. We find a nearly equal mass ratio of $q=0.95 \pm 0.01$ and systemic velocity $\gamma=-0.3 \pm 1.0 \mathrm{~km} \mathrm{~s}^{-1}$.

The large velocity semiamplitude of PYC J11519+0731 Aab implies a short orbital period (at most a few days); therefore the high-energy emission is most likely a result of fast rotation from tidal synchronization rather than youth (Torres et al. 2002; Torres et al. 2003; Shkolnik et al. 2010; Kraus et al. 2011). Further RV and photometric monitoring could provide a spectroscopic orbit and rotationally modulated period, which would give a better indication of whether tidal spin-up could be responsible for the observed activity. 

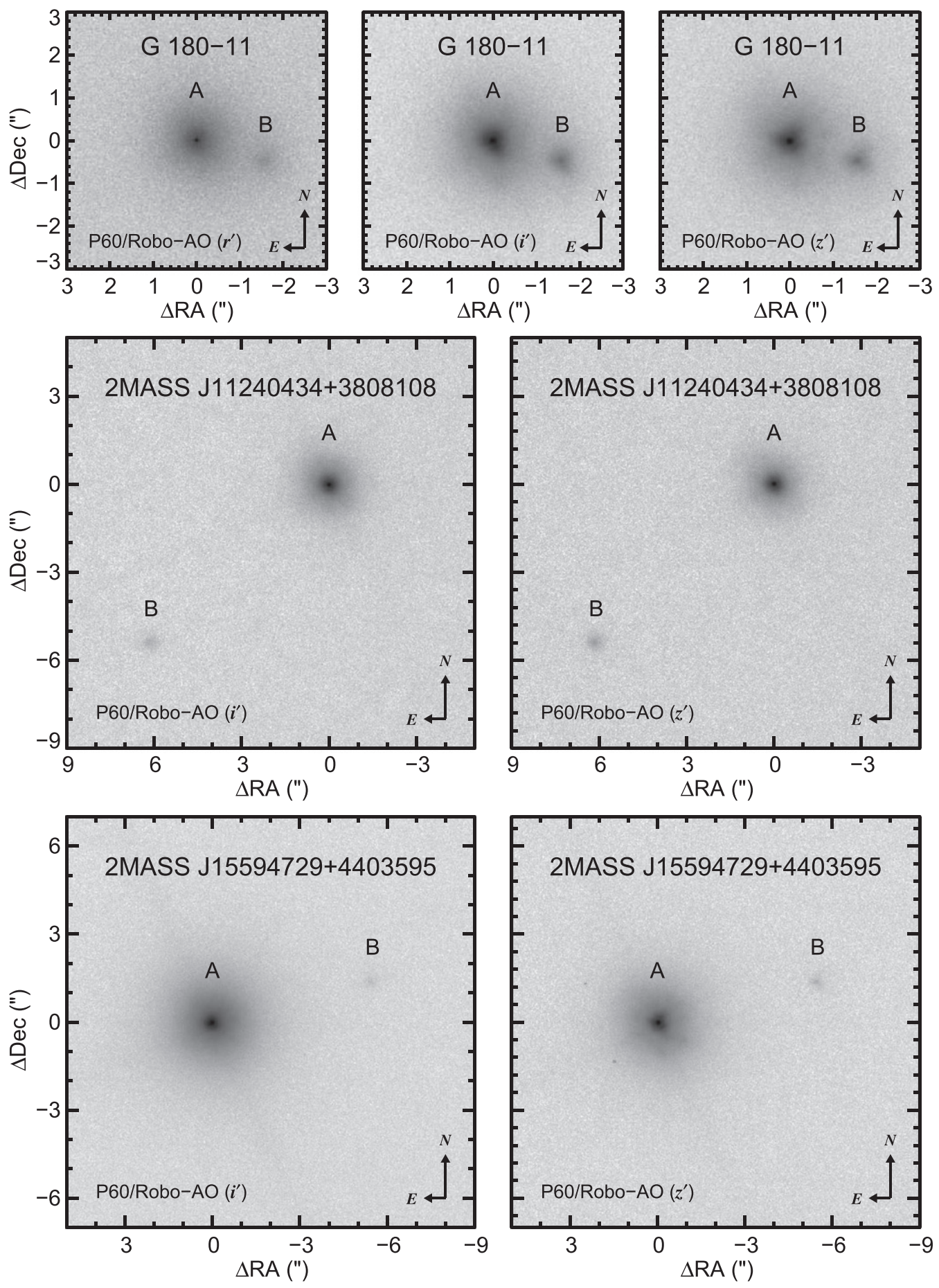

Figure 18. Optical P60/Robo-AO images of G 180-11 AB, 2MASS J11240434+3808108 AB, and 2MASS J15594729+4403595 AB. All three late-M companions are easily detected in $60 \mathrm{~s}$ frames. North is up, and east is left.

\subsubsection{Age and Kinematics of PYC J11519+0731 Aab}

Li I absorption at $6708 \AA$ from either component of PYC J11519+0731 Aab would provide strong, independent evidence of youth. The top panels of Figure 21 show the $\mathrm{Li}$ I region in our three high-resolution spectra. Our HIRES spectrum shows an absorption line that is close to the predicted position of $\mathrm{Li}$ I in the primary. However, in the other epochs there is no convincing detection of $\mathrm{Li} \mathrm{I}$ in either component. At the 12-25 Myr age of the $\beta$ Pic moving group (Yee \& Jensen 2010;
Binks \& Jeffries 2014; Mamajek \& Bell 2014), lithium is not typically detected for spectral types of M2; only at younger ages of $\lesssim 10$ Myr is photospheric lithium still present (Mentuch et al. 2008). The lack of strong lithium in either component sets a lower age limit of $\sim 10 \mathrm{Myr}$ for the system and is therefore consistent with the age of $\beta$ Pic.

Despite lacking a parallax, our systemic RV enables constraints on the kinematics of PYC J11519+0731 Aab. Figure 23 shows the range of $U V W$ heliocentric space velocities (based on the procedure from Johnson \& Soderblom 1987 with 


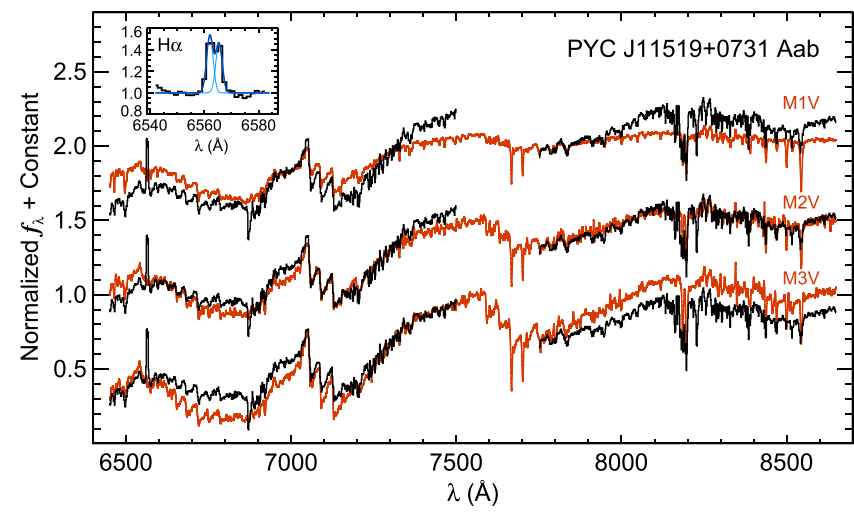

Figure 19. Mayall/RC-spectrograph medium-resolution $(R \approx 2500)$ optical spectrum of PYCJ11519+0731 A (black). Visual classification yields a spectral type of M2, although some peculiarities are evident compared to SDSS templates (red) from Bochanski et al. (2007). The inset shows the $\mathrm{H} \alpha$ emission line, which is marginally resolved here but well-separated in our highresolution spectrum. A joint fit to both components with the AMOEBA downhillsimplex algorithm yields a differential velocity of $\approx 154 \mathrm{~km} \mathrm{~s}^{-1}$. However, for our RV analysis in this work we only include the high-resolution spectra. All spectra are normalized and offset by a constant.
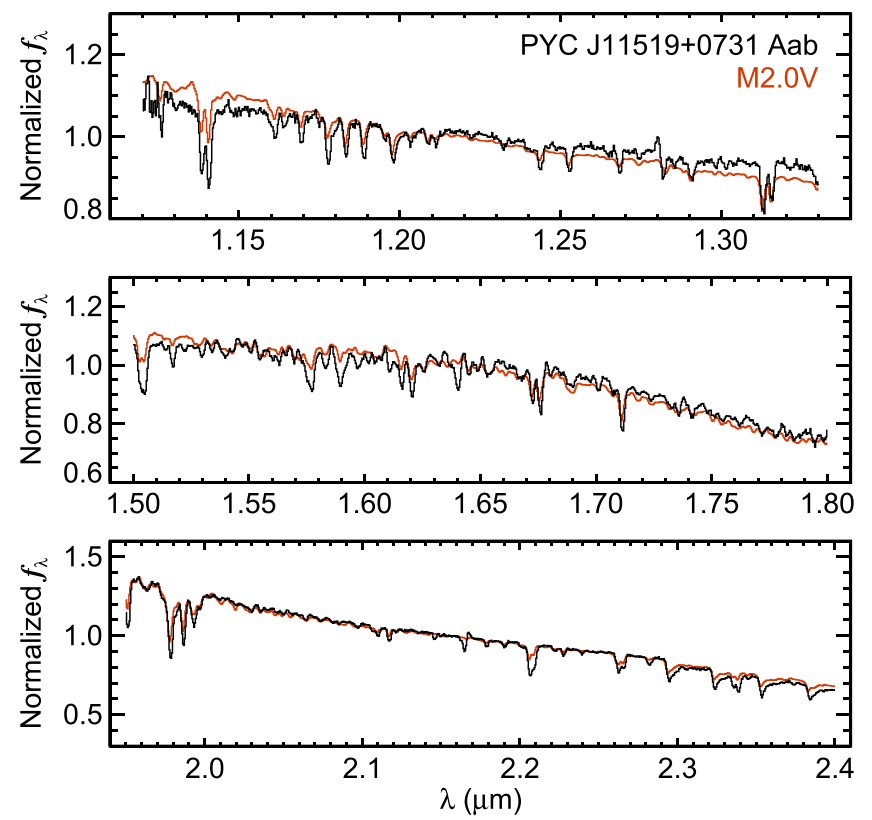

Figure 20. IRTF/SpeX SXD unresolved spectrum of PYC J11519+0731 Aab (black) compared to the M2.0 V template G1 806 (red) from Rayner et al. (2009). Several absorption features in our spectrum are unusually deep, such as the $\mathrm{Mg}_{\mathrm{I}} \lambda 1.503 / 1.505 \mu \mathrm{m}$ and $\mathrm{Si}$ I $\lambda 1.589 \mu \mathrm{m}$ lines, possibly reflecting a higher metallicity. The template is smoothed to the resolution of our spectrum $(R \approx 750)$, and each individual band is normalized.

the coordinate transformation matrix from Murray 1989) and $X Y Z$ galactic positions for PYCJ11519+0731 compared to members of YMGs from Torres et al. (2008). ${ }^{30}$ The systemic $\mathrm{RV}$ we measure $\left(\gamma=-0.3 \pm 1.0 \mathrm{~km} \mathrm{~s}^{-1}\right)$ agrees with the value

\footnotetext{
${ }^{30}$ Note that the proper motion of PYC J11519+0731 is incorrectly listed in several all-sky catalogs. USNO-B (Monet et al. 2003) and the updated PPMXL Catalog (Roeser et al. 2010) list proper motions of $\mu_{x} \cos \delta=-112 \mathrm{mas} \mathrm{yr}^{-1}$ and $\mu_{\delta}=122 \mathrm{mas} \mathrm{yr}^{-1}$ and $\mu_{\alpha} \cos \delta=-126 \mathrm{mas} \mathrm{yr}^{-1}$ and $\mu_{\delta}=110 \mathrm{mas} \mathrm{yr}^{-1}$, respectively. However, visual inspection of older Digitized Sky Survey and 2MASS images clearly shows movement in the southwest direction. We therefore adopt the original PPMXL proper motion of $\mu_{\alpha} \cos \delta=-142 \pm 19$

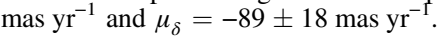

of $-1.5 \pm 1.0 \mathrm{~km} \mathrm{~s}^{-1}$ predicted by Schlieder et al. (2012b) assuming $\beta$ Pic membership, and the range of $U V W$ velocities is consistent with that group for distances between $\sim 20-30 \mathrm{pc}$. However, the physical location of PYC J11519+0731 disagrees from known members in $Z$ by at least 20 pc.

Group membership can be further tested by placing PYC J11519+0731 on a color-magnitude diagram. Because they are still contracting, $\beta$ Pic members are overluminous by $\sim 1 \mathrm{mag}$ in $V$ band compared to field stars on the main sequence (e.g., Riedel et al. 2014). Assuming similar spectral types for PYC $\mathrm{J} 11519+0731 \mathrm{Aa}$ and $\mathrm{Ab}$ and a near-IR flux ratio of $0.6 \pm 0.1$ from our CC analysis (Section 3.11.1), the integrated apparent $V$-band magnitude of $12.5 \pm 0.1 \mathrm{mag}$ from Droege et al. (2006) can be decomposed into individual $V$-band magnitudes of $13.01 \pm 0.12 \mathrm{mag}$ and $13.57 \pm 0.16 \mathrm{mag}$ for Aa and Ab. At a distance of $20 \mathrm{pc}(30 \mathrm{pc})$, these correspond to absolute magnitudes of $11.50 \pm 0.12 \mathrm{mag}(10.62 \pm 0.12 \mathrm{mag})$ and $12.07 \pm 0.16 \mathrm{mag}(11.19 \pm 0.16 \mathrm{mag})$ for $\mathrm{Aa}$ and $\mathrm{Ab}$, respectively. At the system $V-K$ color of $4.6 \pm 0.1 \mathrm{mag}, \beta$ Pic members are expected to have $M_{V} \approx 9.5 \mathrm{mag}$ (Riedel et al. 2014). Instead, both components of PYC J11519 +0731 Aab appear consistent with the main sequence given the range of plausible distances assuming group membership. It is therefore likely this system is simply an old but active interloper with (partially constrained) kinematics similar to the $\beta$ Pic YMG.

\subsubsection{PYC J11519+0731 B}

In 2012 we imaged PYC J11519+0731 as part of our PALMS survey. As expected, the close SB2 pair PYC J11519 +0731 Aab was not resolved in our NIR images, implying a separation less than about one PSF FWHM $(40.4 \pm 0.8$ mas in $H$ band, or $\approx 1.5 \mathrm{AU}$ at $37 \mathrm{pc})$. Indeed, two $0.2 M_{\odot}$ stars on circular, edge-on orbits would need to reside at separations of $0.03 \mathrm{AU}$ to match the maximum observed RV semiamplitude. We did, however, identify a moderate-contrast $(\Delta H=$ $5.4 \pm 0.2 \mathrm{mag}$ ) point source located at $\approx 0.5^{\prime \prime}$ from PYC $\mathrm{J} 11519+0731 \mathrm{Aab}$ and immediately obtained unsaturated frames in $Y, J, H, K_{S}$, and $L^{\prime}$ (Figure 24 and Table 3). The companion's red colors point to a cool temperature and late-M spectral type. To search for additional companions in the system we obtained deeper $H$-band coronagraphic images in 2013 (20 frames each with $30 \mathrm{~s}$ integration times) in ADI mode.

A single image in our ADI sequence is shown alongside the PSF-subtracted and coadded version using the entire sequence in Figure 25. The companion is visible in the single image and is similar in appearance to the quasi-static speckles but stands out prominently with a peak S/N of 370 in our PSF-subtracted image. The PSF subtraction was carried out in a similar fashion as the deep imaging in Bowler et al. (2015), except here we have made use of 100 reference PSF images for our LOCI reduction (Lafrenière et al. 2007) from a PSF library of over 2000 registered coronagraphic images from our PALMS survey. Reference images were selected as having the lowest residuals between $0.3^{\prime \prime}$ and $2^{\prime \prime}$ after scaling and subtracting the library template to each science image. Local masking was applied at the location of PYC J11519+0731 B to minimize biasing the LOCI coefficients.

We obtained three additional epochs of astrometry with Keck/NIRC2 between 2012 and 2014 and one with Gemini-S/ NICI in 2013 (Table 3). Compared to the expected motion of a 

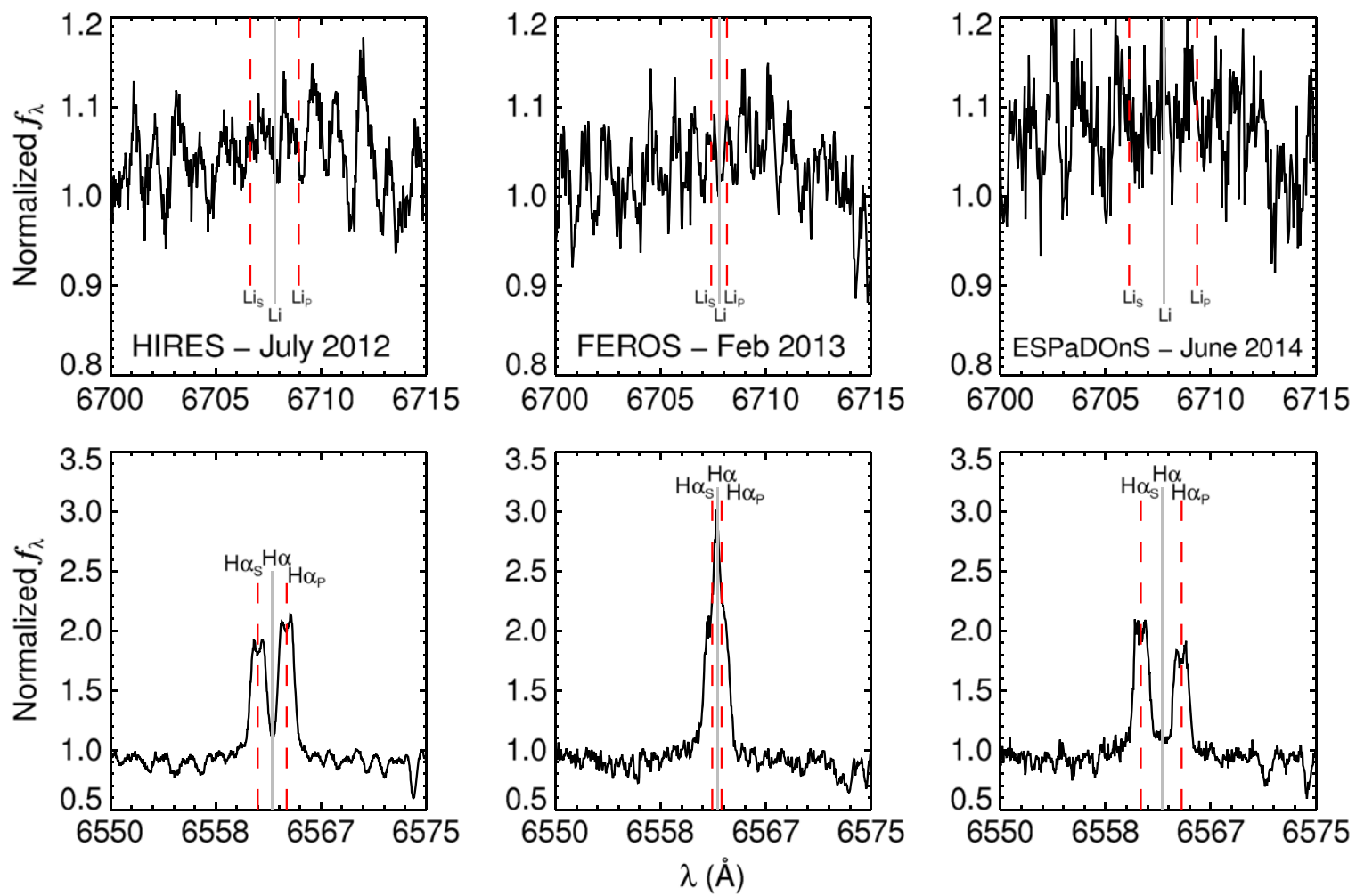

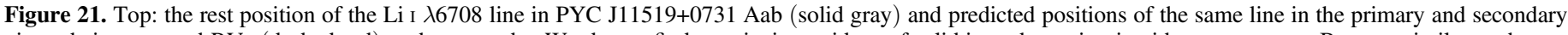

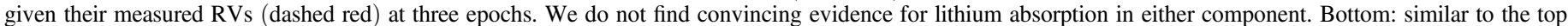

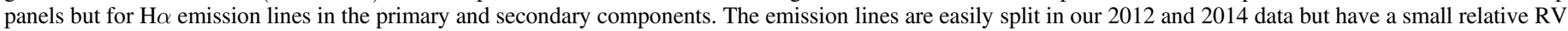
in our 2013 data set.

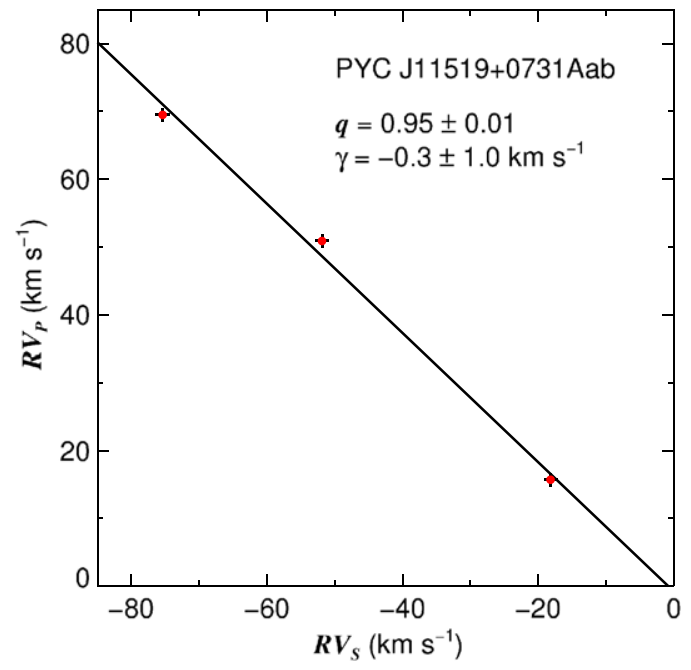

Figure 22. Radial velocities of PYC J11519+0731 Aa and Ab. Our three epochs enable a measurement of the mass ratio $(q)$ and systemic velocity $(\gamma)$ for the system. The negative slope of the best-fit line (solid line) is equal to the mass ratio of the system $(0.95 \pm 0.01)$, and we measure a systemic $\mathrm{RV}$ of $-0.3 \pm 1.0 \mathrm{~km} \mathrm{~s}^{-1}$.

stationary object (Figure 26), our astrometry unambiguously shows that PYC J11519+0731 B is comoving with the primary Aab. We also find clear signatures of orbital motion. The reduced chi-squared $\left(\chi_{\nu}^{2}\right)$ value of a constant (linear) fit to the separation measurements is 7.7 (1.0), and a constant (linear) fit to the PA is 268 (3.5). The linear fits correspond to changes of $+9.7 \pm 1.2$ mas yr$^{-1}$ and $-2.04 \pm 0.04 \mathrm{yr}^{-1}$ for the separation and PA, respectively. Curvature in the orbit should be evident within a few years.

Our $K$-band Keck/OSIRIS spectrum of PYC J11519+0731 B is shown in Figure 27 compared to field templates from the IRTF Spectral Library (Cushing et al. 2005; Rayner et al. 2009) and low-gravity (VL-G) templates from Allers \& Liu (2013). PYC J11519+0731 B appears most similar to the field M8 template in overall shape and the depth of CO and $\mathrm{Na}$ I features. The $2.29 \mu \mathrm{m} \mathrm{CO}$ absorption is weaker, and the $\sim 2 \mu \mathrm{m}$ steam absorption is stronger in the very low gravity objects compared to PYC J11519+0731 B. In addition, the $2.207 \mu \mathrm{m}$ $\mathrm{Na}$ I doublet, which is sensitive to surface gravity (and hence age), is weaker in all very low gravity templates. This is perhaps the strongest indication that PYC J11519+0731 B is older than 30 Myr. Because of the limited spectral coverage, we adopt a spectral type of M8 with uncertainty of \pm 1 subtype.

The mass of PYC J11519+0731 B depends on the distance and, more importantly, on the system age. The photometric distance to PYCJ11519+0731 B using the $H$-band absolute magnitude-spectral-type relations from Dupuy \& Liu (2012) is $37 \pm 6 \mathrm{pc}$. This incorporates uncertainties in the photometry, spectral classification, and rms of the relation. Using the $H$-band bolometric correction from Liu et al. (2010a) we infer a luminosity of $\log L / L_{*}=-3.35 \pm 0.16$ dex. At ages of $\{21 \pm 2 \mathrm{Myr}, 100 \pm 10 \mathrm{Myr}, 1.0 \pm 0.1 \mathrm{Gyr}$, and $5.0 \pm 0.5 \mathrm{Gyr}\}$, the evolutionary models of Burrows et al. (1997) imply masses of $\{15.3 \pm 1.3, \quad 42 \pm 7, \quad 89 \pm 5, \quad$ and $90 \pm 4\} \quad M_{\text {Jup }}$ for PYC J11519+0731 B. If the system is younger than $\approx 350 \mathrm{Myr}$, then the companion mass lies below the hydrogen-burning limit.

No other candidate companions were identified in our deep imaging, which extends out to $\approx 4^{\prime \prime}$ for complete azimuthal 

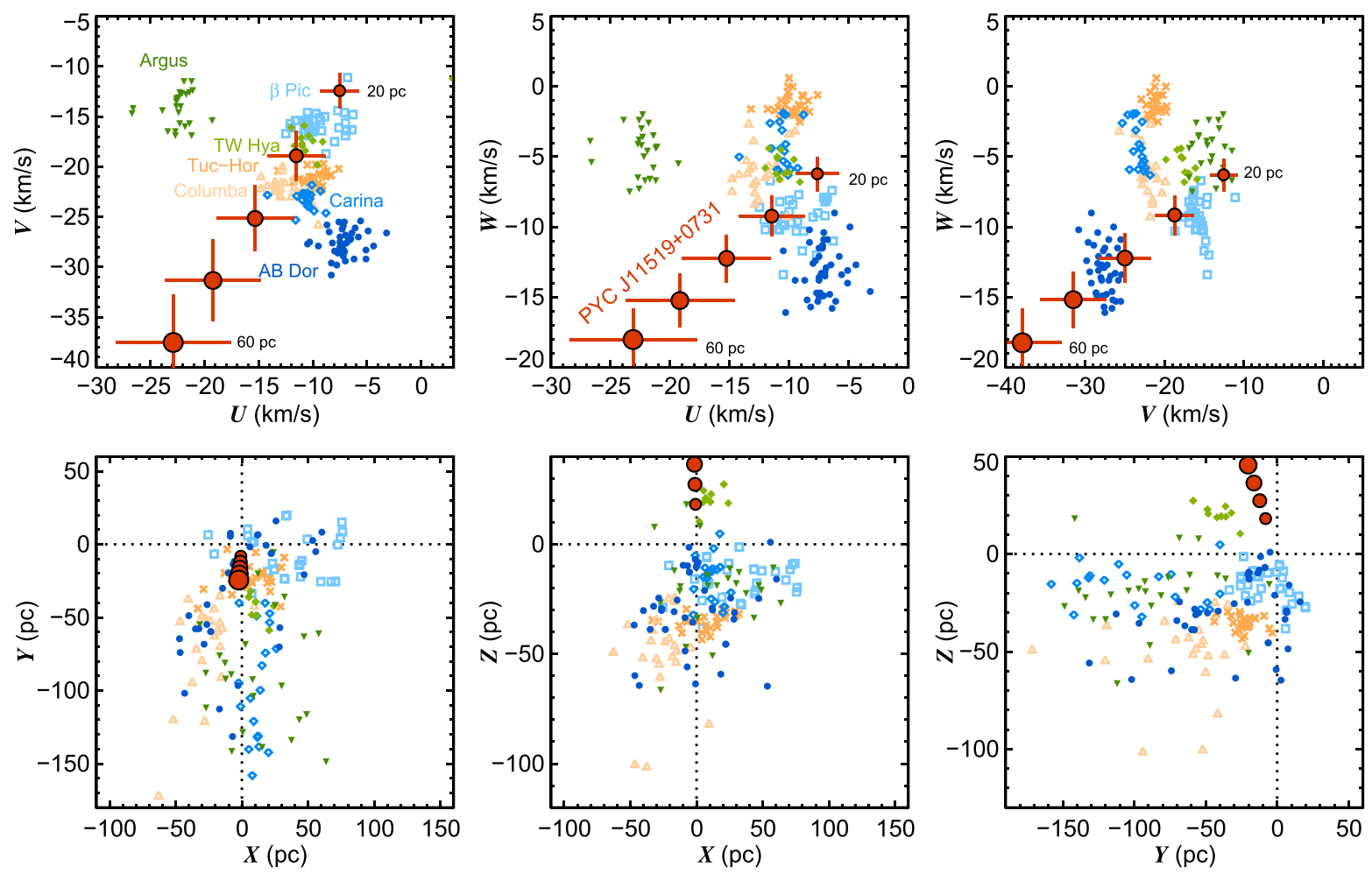

Figure 23. Partially constrained $U V W$ kinematics and $X Y Z$ space positions of PYC J11519+0731 Aab compared to young moving group members from Torres et al. (2008). Red circles show the stars' position for distances of 20,30, 40, 50, and 60 pc based on the systemic RV. At close distances of $\approx 20-30$ pc, PYC J11519 +0731 Aab is consistent with $\beta$ Pic moving group, but physically would be tens of parsecs from known members.

sensitivity and to $\approx 8^{\prime \prime}$ for partial sensitivity. The $7 \sigma H$-band contrast curve shown in Figure 28 is measured by computing the rms in 5 pixel (50 mas) annuli, as described in Bowler et al. (2015). Unsaturated frames are used at separations below $0.3^{\prime \prime}$, and our deep ADI data are used at larger radii. Our final contrast curve in $\Delta H$ and FOV coverage (in brackets) is $\{4.0$, 5.4, 10.6, 12.2, 13.1, 13.8, 13.4, 14.0,14.2, 14.0, and 13.4\} mag for separations of $\left\{0.1^{\prime \prime}, 0.2^{\prime \prime}, 0.5^{\prime \prime}, 0.75^{\prime \prime}, 1.0^{\prime \prime}, 1.5^{\prime \prime}, 2^{\prime \prime}\right.$, $3^{\prime \prime}, 4^{\prime \prime}[1.0], 5^{\prime \prime}[0.69]$, and $\left.8^{\prime \prime}[0.05]\right\}$. That is, we are fully sensitive to spatial coverage within $\approx 4^{\prime \prime}$, but are only sensitive to a fraction of the sky beyond that, owing to the square detector. At ages of $\{21 \mathrm{Myr}, 100 \mathrm{Myr}, 1 \mathrm{Gyr}$, and $5 \mathrm{Gyr}\}$, we remain highly sensitive $(>90 \%)$ to companions with masses as low as $\{1,2,10$, and 25$\} M_{\mathrm{Jup}}$ at $100 \mathrm{AU}$ based on the evolutionary models of Baraffe et al. (2003).

\subsection{G 180-11 AB (2MASS J15553178+3512028 AB)}

A $1.6^{\prime \prime}$ companion to the M4.0 \pm 0.5 star G 180-11 (also known as GJ 3928) was first discovered by McCarthy et al. (2001) and confirmed to be physically bound by Daemgen et al. (2007), Law et al. (2008), and Janson et al. (2012). Unresolved detections in ROSAT and GALEX together with strong $\mathrm{H} \alpha$ emission $(E W \approx-6$ to $-8.1 \AA$; Riaz et al. 2006; Shkolnik et al. 2009) indicate the primary and/or companion are active. Riaz et al. (2006) found a saturated fractional X-ray luminosity of $\log L_{X} / L_{\mathrm{bol}}=-2.98 \mathrm{dex}$ for the system, pointing to a relatively young age. Hartman et al. (2011) measured a rotational period of 3.5209 days as part of the HATNet survey.

Recently, Dittmann et al. (2014) measured a parallactic distance of $28 \pm 3 \mathrm{pc}$ to $\mathrm{G} 180-11 \mathrm{AB}$ as part of the MEarth transit survey. At this distance, the primary-companion separation is $45 \mathrm{AU}$. Together with the $\mathrm{RV}$ of $-15.5 \pm 0.7 \mathrm{~km} \mathrm{~s}^{-1}$ from Shkolnik et al. (2012), this corresponds to $U V W$ kinematics of $\{-35 \pm 3,-17.6 \pm 1.4$, and $8 \pm 2\} \mathrm{km} \mathrm{s}^{-1}$ and $X Y Z$ space positions of $\{9.9 \pm 1.1,14.9 \pm 1.6$, and $21.5 \pm 2\}$ pc. Malo et al. $(2013,2014)$ find that $\mathrm{G} 180-11 \mathrm{AB}$ is a likely member of the Argus moving group based on the RV alone, which matches the predicted value assuming group membership, but the parallactic distance disagrees with the statistical distance of $13 \pm 1 \mathrm{pc}$, so the similar RV is probably coincidental. Indeed, the kinematics of G 180-11 AB do not appear to be consistent with any known moving groups.

The pair are easily resolved with our Keck/NIRC2 and P60/ Robo-AO observations spanning 0.6-2.2 $\mu \mathrm{m}$ (Figure 18). Compared with previous astrometry for this system (see Janson et al. 2012 for a summary), our new data show slight outward motion by $\approx 6$ mas $\mathrm{yr}^{-1}$ and P.A. change of $\approx 0.8^{\circ} \mathrm{yr}^{-1}$. The orbital motion is quite slow, so it will take decades to complete an appreciable portion of its orbit.

Daemgen et al. (2007) infer a spectral type of $\mathrm{M} 7 \pm 2$ for G 180-11 B from its $K$-band contrast ratio, Cruz et al. (2003) deduce an $\sim$ M6 classification, and Law et al. (2008) estimate a spectral type of M6.5 from its optical colors. In 2013 we obtained resolved NIR $(J H K)$ spectra of G 180-11 B with Keck/OSIRIS. Compared to templates from the IRTF Spectral Library (Rayner et al. 2009) in Figure 29, G 180-11 B best resembles M5-M6 field objects from 1.2-2.4 $\mu \mathrm{m}$ and in individually normalized $J, H$, and $K$ bands with a slightly better match to the M6 spectrum. We therefore adopt a NIR spectral type of $\mathrm{M} 6.0 \pm 0.5$, in agreement with previous estimates from colors. Although the $2.21 \mu \mathrm{m} \mathrm{Na}$ I doublet line is equally strong in G $180-11 \mathrm{~B}$ as in the field templates, the 1.244 and $1.253 \mu \mathrm{m} \mathrm{K}$ I lines are noticeably weaker, pointing to a low surface gravity in line with an age younger than $\sim 200$ Myr. 

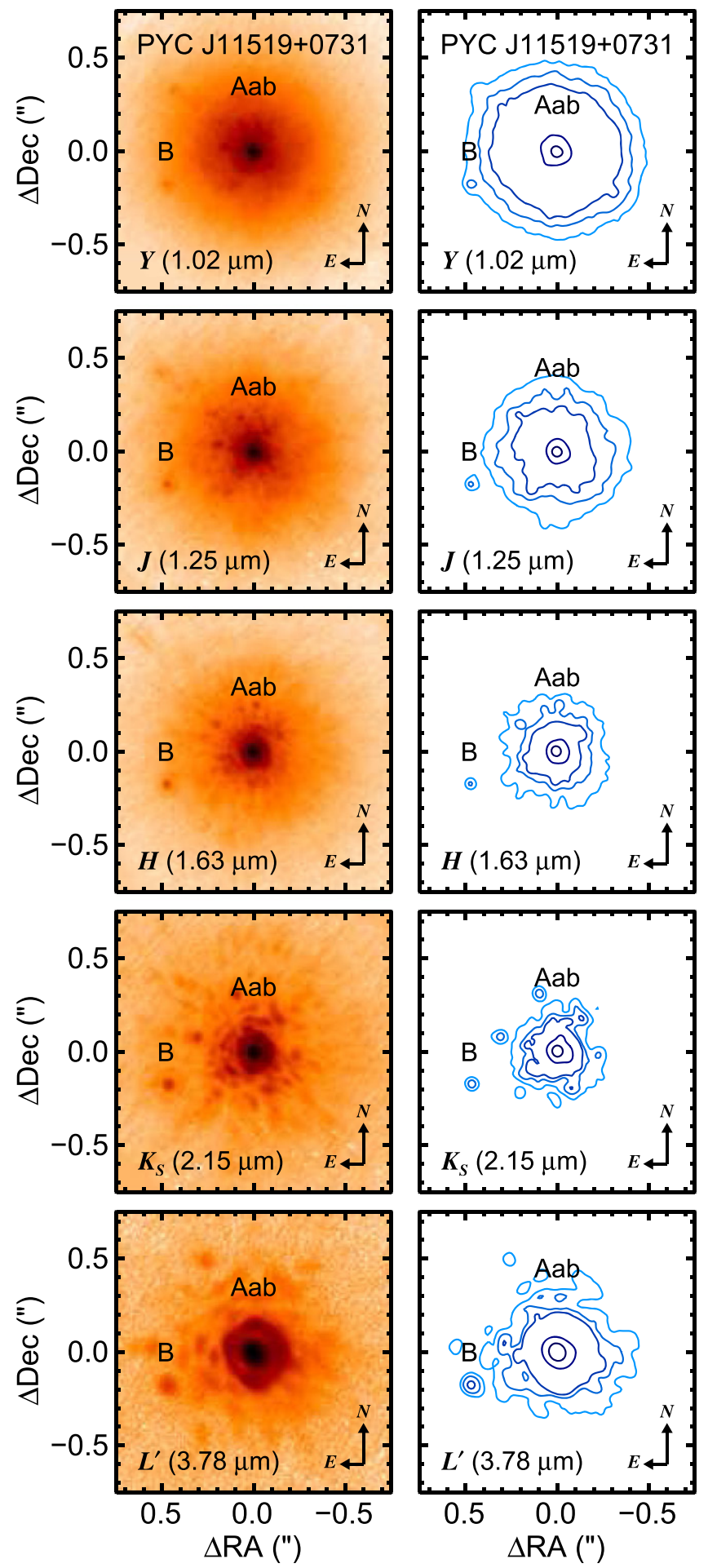

Figure 24. Keck/NIRC2 $Y$-, $J-, H-, K_{S^{-}}$, and $L^{\prime}$-band AO images of PYC $\mathrm{J} 11519+0731$. The $0.5^{\prime \prime}$ companion PYC J11519+073 B is clearly visible in these unsaturated coadded frames. Contours represent $0.3 \%, 0.6 \%, 1 \%, 10 \%$, and $50 \%$ of the peak flux after convolution with a Gaussian kernel with a FWHM equal to that of the image PSF. North is up, and east is left. The field of view is $1.5^{\prime \prime} \times 1.5$ !"

Based on the parallactic distance and an $\mathrm{H}$-band bolometric correction from Liu et al. (2010a), we measure a luminosity of $\log L / L_{\odot}=-2.48 \pm 0.13 \mathrm{dex}$ for $\mathrm{G} 180-11 \mathrm{~B}$. At ages of $50 \pm 5 \mathrm{Myr}, 100 \pm 10 \mathrm{Myr}$, and $200 \pm 20 \mathrm{Myr}$, this corresponds to masses of $88 \pm 18 M_{\text {Jup }}, 120 \pm 20 M_{\text {Jup }}$, and $150 \pm 20 M_{\text {Jup }}$ using the Burrows et al. (1997) evolutionary models. Unless this system is younger than $\sim 40 \mathrm{Myr}$, $\mathrm{G} 180-11 \mathrm{~B}$ is a low-mass star instead of a brown dwarf.

\subsection{MASS J15594729+4403595 AB}

2MASS J15594729+4403595 is a young active early-M dwarf with a comoving substellar companion first identified by Janson et al. (2012, see Bowler et al. 2015 for details) at a projected separation of 5.6" (150 AU). The companion was easily recovered with our Robo-AO imaging in $i^{\prime}$ and $z^{\prime}$ bands (Figure 18). Our SNIFS 4000-9000 A optical spectrum of the primary in Figure 1 reveals weak $\mathrm{H} \alpha$ emission $(\mathrm{EW}=-2.3 \AA)$ and is well-matched with an M1.5 V spectrum, created by averaging the M1 and M2 templates from Bochanski et al. (2007). Our own RV of $-19.6 \pm 0.6 \mathrm{~km} \mathrm{~s}^{-1}$ from ESPaDOnS disagrees with the value of $-15.8 \pm 0.5 \mathrm{~km} \mathrm{~s}^{-1}$ from Malo et al. (2014) by $4 \mathrm{~km} \mathrm{~s}^{-1}$, a $4.9 \sigma$ difference (Table 6), raising the possibility that the primary is an SB1. Although the system does not appear to coincide with any known moving groups, the intermediate-gravity NIR spectrum of 2MASS J15594729 +4403595 B implies a young age of $\approx 50-200$ Myr (Bowler et al. 2015).

Our spatially resolved Keck/ESI optical spectrum of 2MASS J15594729+4403595 B is shown in Figure 30. 2MASS J15594729+4403595 B exhibits modest $\mathrm{H} \alpha$ emission $(\mathrm{EW}=-6.4 \AA)$ and absorption features typical of late-M dwarfs. Compared to templates from Bochanski et al., 2MASS J15594729+4403595 B fits the M7.0 and M7.5 objects reasonably well. The index-based classification from Hammer (Covey et al. 2007) is M7. Altogether we adopt an optical classification of M7.5 \pm 0.5 . Note, however, that the best match to the entire $0.5-2.5 \mu \mathrm{m}$ spectrum is slightly later (M8; Figure 31), which is common with visual classification of young brown dwarfs (Allers \& Liu 2013).

As shown in Figure 31, we find strong Li I $\lambda 6708$ absorption in the companion $(\mathrm{EW}=0.71 \AA)$. The detection of lithium provides independent constraints on the mass and age of the companion through the "lithium test" (Rebolo et al. 1992; Rebolo et al. 1996; Kirkpatrick 2008). Basri (1998) shows that an object with Li I absorption with a luminosity below $\log \left(L / L_{\odot}\right)=-3.05$ dex or $T_{\text {eff }}<2670 \mathrm{~K}$ (spectral type $\gtrsim$ M7) is unambiguously substellar and young ( $\lesssim 200 \mathrm{Myr})$. This is consistent with the intermediate surface gravity and mass of $43 \pm 9 M_{\text {Jup }}$ for 2MASS J15594729+4403595 B found in Bowler et al. (2015).

\subsection{GJ 4378 Aal2b (2MASS J23572056-1258487 Aa12b)}

\subsubsection{The Wide Binary GJ 4378 A and GJ 4379 B}

GJ 4378 A (LP 704-15, G 273-186) and GJ 4379 B (2MASS J23571934-1258406, G 273-185, LP 704-14) form a pair of comoving mid-M dwarfs separated by $20^{\prime \prime}$ (Giclas et al. 1975). Both stars have similar reported $V$-band (13.0 mag from APASS/UCAC4, Zacharias et al. 2013) and $r^{\prime}$-band (12.4 mag from CMC15, Muiños \& Evans 2014) magnitudes, but GJ $4378 \mathrm{~A}$ is brighter in $J H K$ by $\sim 0.5 \mathrm{mag}$ (from 2MASS, Skrutskie et al. 2006). GJ 4378 A is classified by Reid et al. (2004, 1995) and Riaz et al. (2006) as M4, M4, and M3, respectively, from optical spectroscopy. The same studies classify GJ 4379 B as M4, M3, M4. We find similar spectral types of M3.5 for GJ 4379 B and M4.5 for GJ 4378 A from our own lowresolution optical spectra (Figure 1). Although GJ 4378 A is slightly brighter, its spectral type is slightly later. Our moderate- 


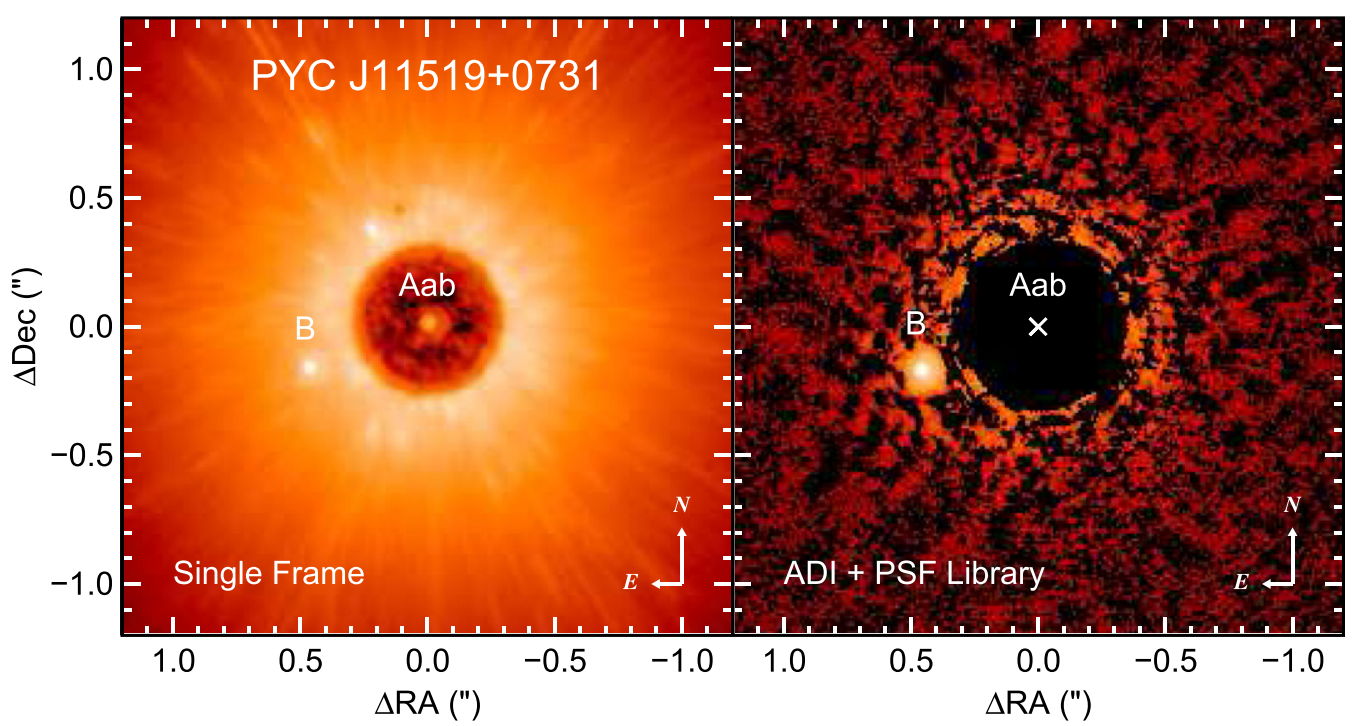

Figure 25. NIRC2 $H$-band coronagraphic observations of PYC J11519+0731 AabB. The Aab component, unresolved here, is centered behind the $\approx 0.1 \%$-throughput 600 mas diameter coronagraph. The image on the left is a single frame in our ADI sequence; PYC J11519+0731 B $(\Delta H=5.2 \mathrm{mag})$ is visible but resembles speckle noise. The right panel shows the result after PSF subtraction with a library of NIRC2 PSF reference images. North is up, and east is left.

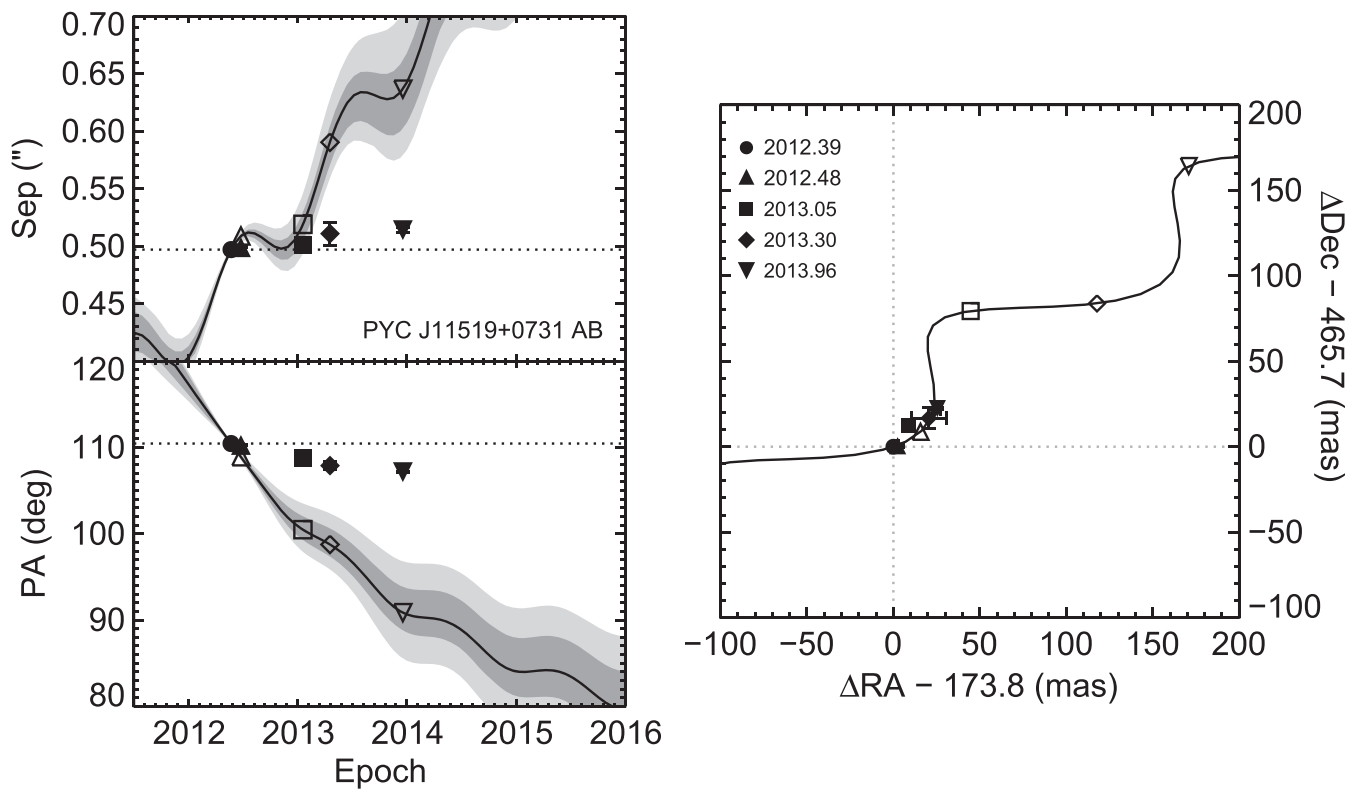

Figure 26. Relative astrometry of PYC J11519+0731 Aab and B between 2012 and 2014 (filled symbols). The solid curve shows the expected path of a background object, and gray-shaded regions show $1 \sigma$ and $2 \sigma$ confidence intervals. Open symbols represent the predicted astrometry for a stationary object at the epochs of the observations. The pair is clearly comoving and exhibits slight but significant orbital motion.

resolution SOAR spectra of the pair are shown in Figure 3. GJ 4378 A exhibits $\mathrm{H} \alpha$ emission $(\mathrm{EW}=-4.5 \AA$ ), whereas none is apparent in the companion GJ $4379 \mathrm{~B}$.

The system coincides with a ROSAT Bright Source Catalog detection (1RXS J125720.0-125852), but it is unclear which component is the counterpart because of the ROSAT PSPC's large positional uncertainty of $\sim 13^{\prime \prime}(1 \sigma$; Voges et al. 1999). GJ $4378 \mathrm{~A}$ was detected in both GALEX in FUV and NUV bandpasses, whereas GJ 4379 B was detected in NUV alone. The NUV $-J$ color, which traces UV excess from chromospheric emission, is $10.58 \pm 0.05 \mathrm{mag}$ and $13.17 \pm 0.19 \mathrm{mag}$ for the $\mathrm{A}$ and B, respectively. The blue NUV $-J$ of GJ $4378 \mathrm{~A}$ is consistent with activity levels of YMG members (10-150 Myr), but the red color of GJ 4379 B is more consistent with older M dwarfs in the Hyades and the field (Rodriguez et al. 2013).
Malo et al. (2013) identify GJ 4378 A as a candidate member of the $\sim 40$ Myr Argus association based on its proper motion and activity. Assuming group membership, they predict a kinematic distance of $25 \pm 1 \mathrm{pc}$ and $\mathrm{RV}$ of $-1.0 \pm 0.8 \mathrm{~km} \mathrm{~s}^{-1}$. In 2009 we obtained echelle spectroscopy of GJ 4378 A and GJ $4379 \mathrm{~B}$ with the du Pont telescope. CC analysis of our du Pont spectrum of GJ 4378 A shows it is a near-equal flux SB2 with component velocities of $-7.0 \pm 0.8 \mathrm{~km} \mathrm{~s}^{-1}$ for the primary (GJ 4378 Aa1) and $49.2 \pm 1.1 \mathrm{~km} \mathrm{~s}^{-1}$ for the companion (GJ 4378 Aa2). ${ }^{31}$ Assuming the pair are equal mass, the

\footnotetext{
31 To avoid confusion, we adopt the following nomenclature for this hierarchical quadruple system: we retain the names of the $20^{\prime \prime}$ pair GJ $4378 \mathrm{~A}$ and GJ $4378 \mathrm{~B}$, GJ $4378 \mathrm{~A}$ is a $\approx 0.5^{\prime \prime}$ visual binary (see 3.14.2) with GJ 4378 Aa and GJ 4378 Ab components, and GJ 4378 Aa is an SB2 with components GJ $4378 \mathrm{Aa} 1$ and GJ $4378 \mathrm{Aa} 2$.
} 


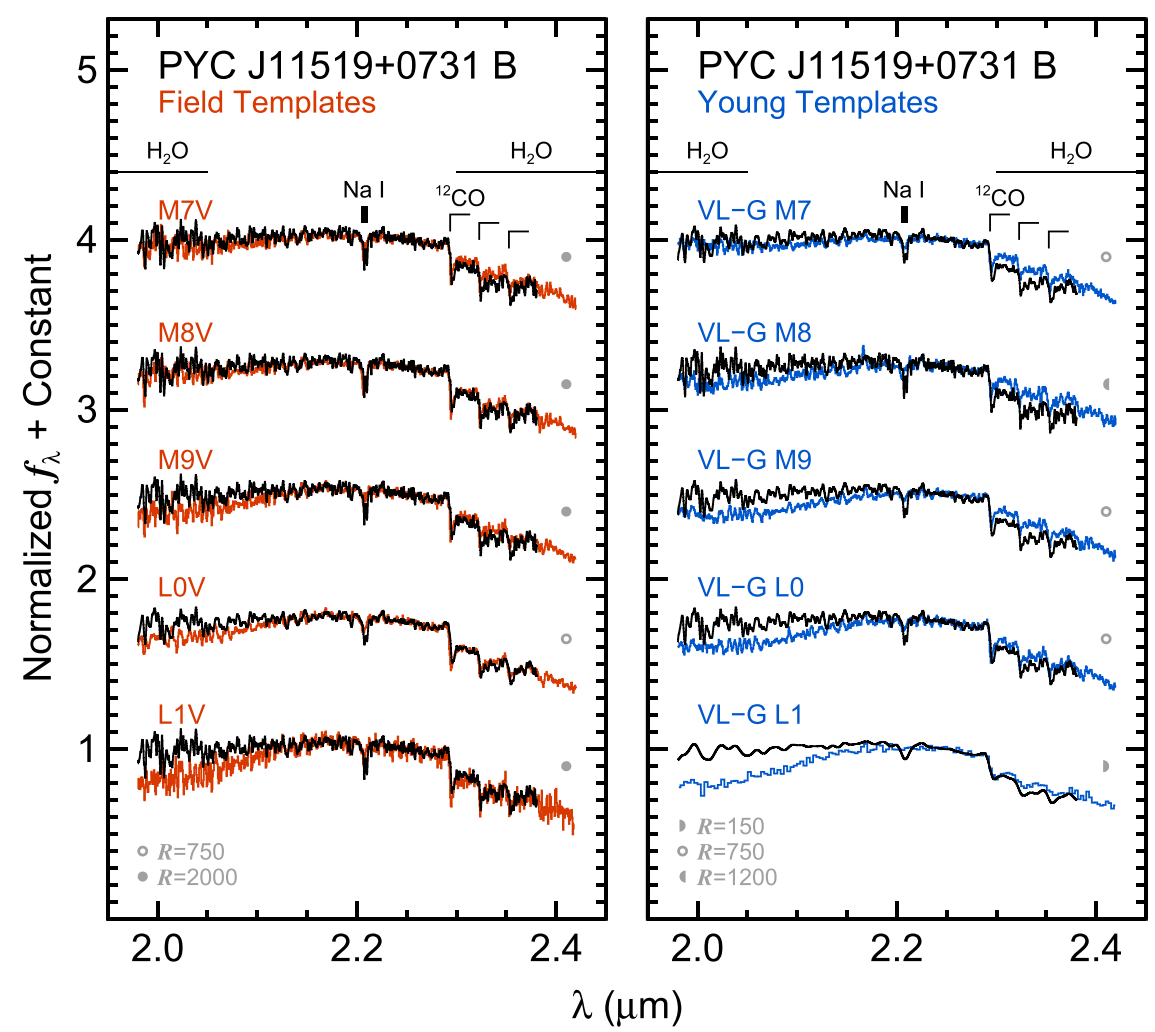

Figure 27. Keck/OSIRIS $K$-band spectrum of PYC J11519+0731 B (black). Compared to field templates (red) from the IRTF Spectral Library (left panel), PYC J11519+0731 B best resembles the M8 object, so we adopt a near-infrared spectral type of M8 \pm 1 . The right panel shows a comparison to "very low gravity" templates (blue) from Allers \& Liu (2013), none of which match our spectrum as well as the high-gravity counterparts. Our spectrum has been Gaussian-smoothed to match the resolving power of the comparison object. All spectra are normalized and offset by a constant.
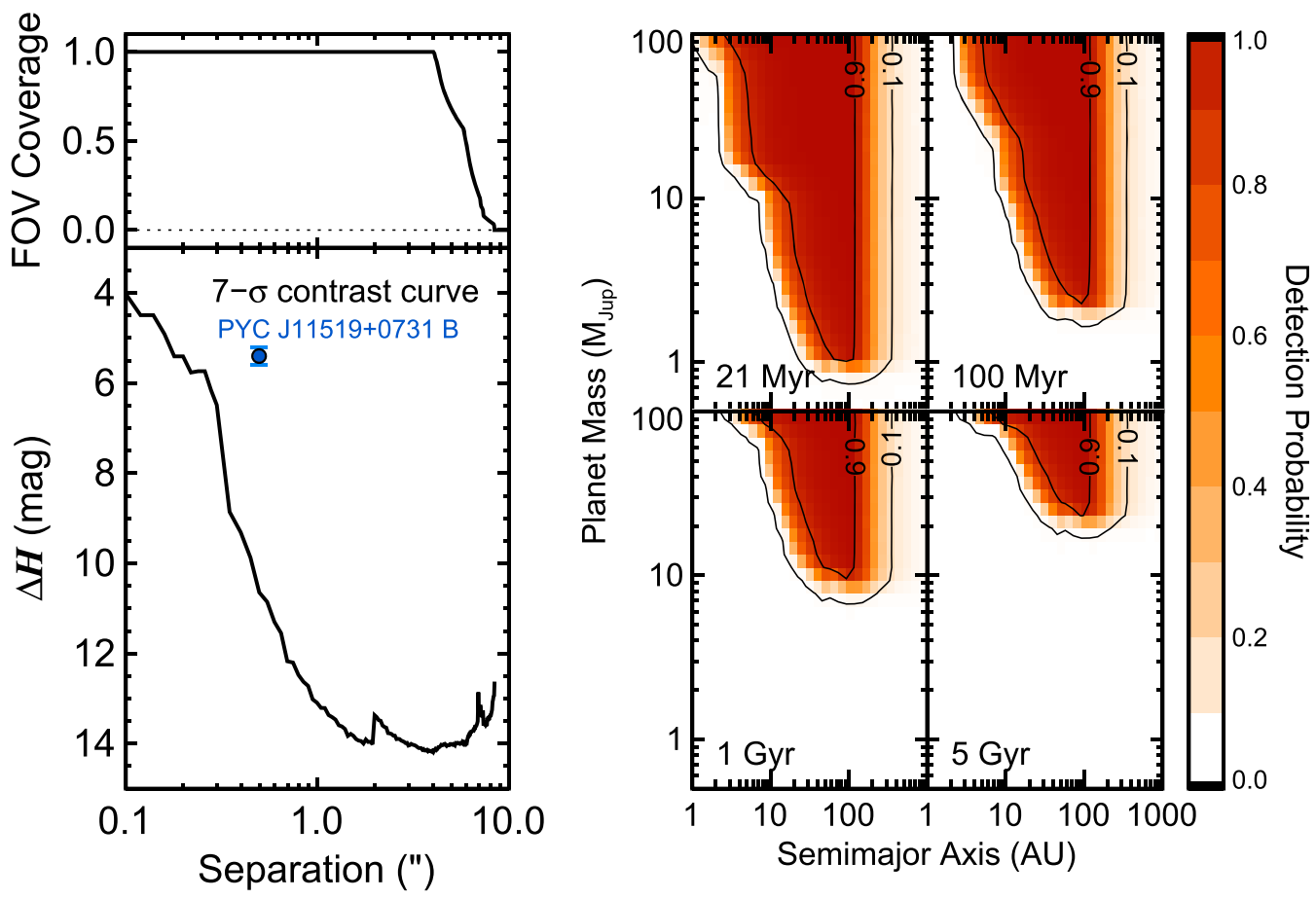

Figure 28. $7 \sigma$ contrast curve (left) and companion sensitivity map (right) for the PYC J11519+0731 system. Our limiting contrast of $\Delta H \approx 13$ mag at $1^{\prime \prime}$ corresponds to planetary masses $\left(\lesssim 13 M_{\text {Jup }}\right)$ at young ages $(\lesssim 1 \mathrm{Gyr})$ and masses in the brown dwarf regime for ages beyond $\sim 1$ Gyr. The conversion from contrast to planet mass assumes hot start (Cond) evolutionary models from Baraffe et al. (2003) and circular orbits. See Bowler et al. (2015) for details. 

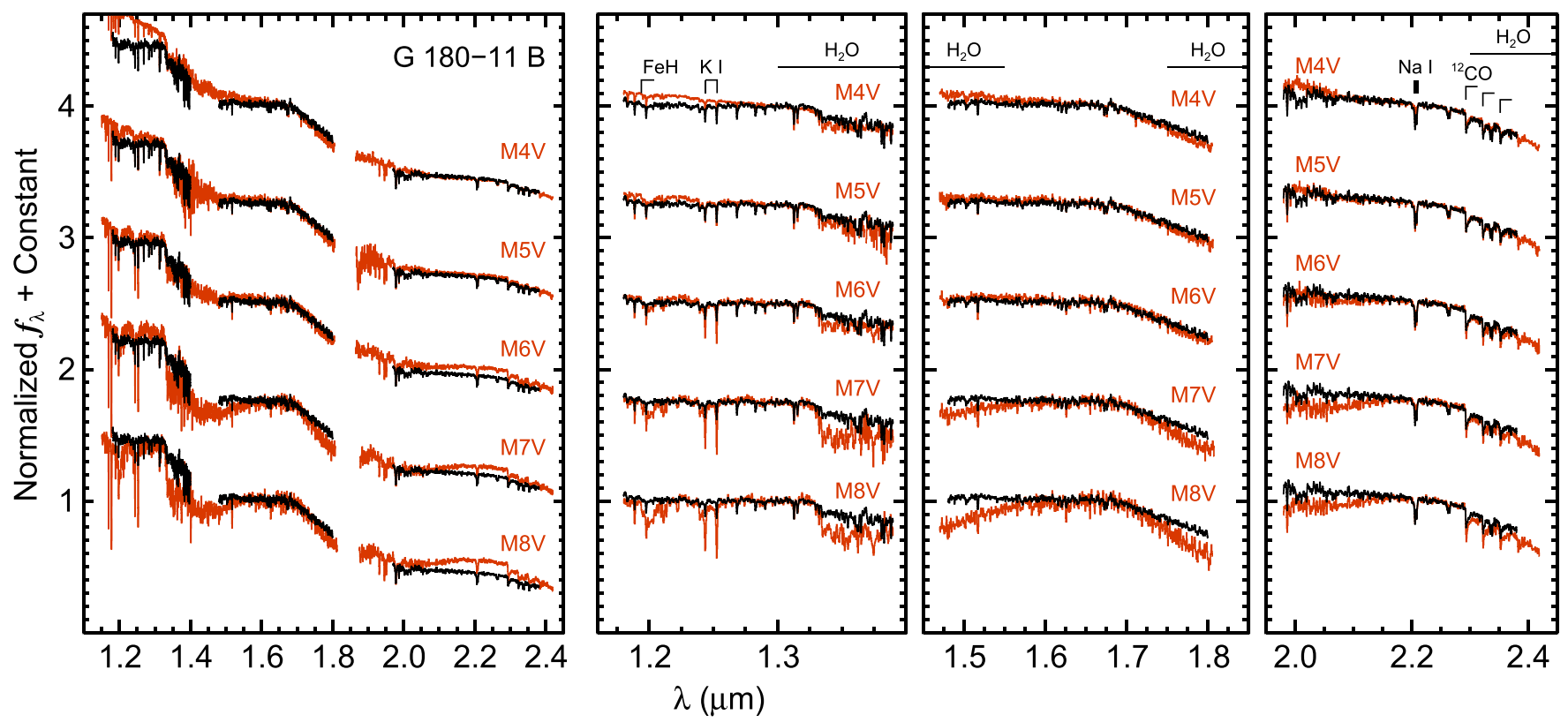

Figure 29. Keck/OSIRIS 1.2-2.4 $\mu \mathrm{m}$ spectrum of G 180-11 B (black). The best match is to M5-M6 field templates (red) from the IRTF Spectral Library. We adopt a near-infrared spectral type of M6 \pm 0.5 . Our OSIRIS spectrum has been Gaussian-smoothed to match the resolving power of the templates $(R \approx 2000)$, and all spectra are normalized and offset by a constant.

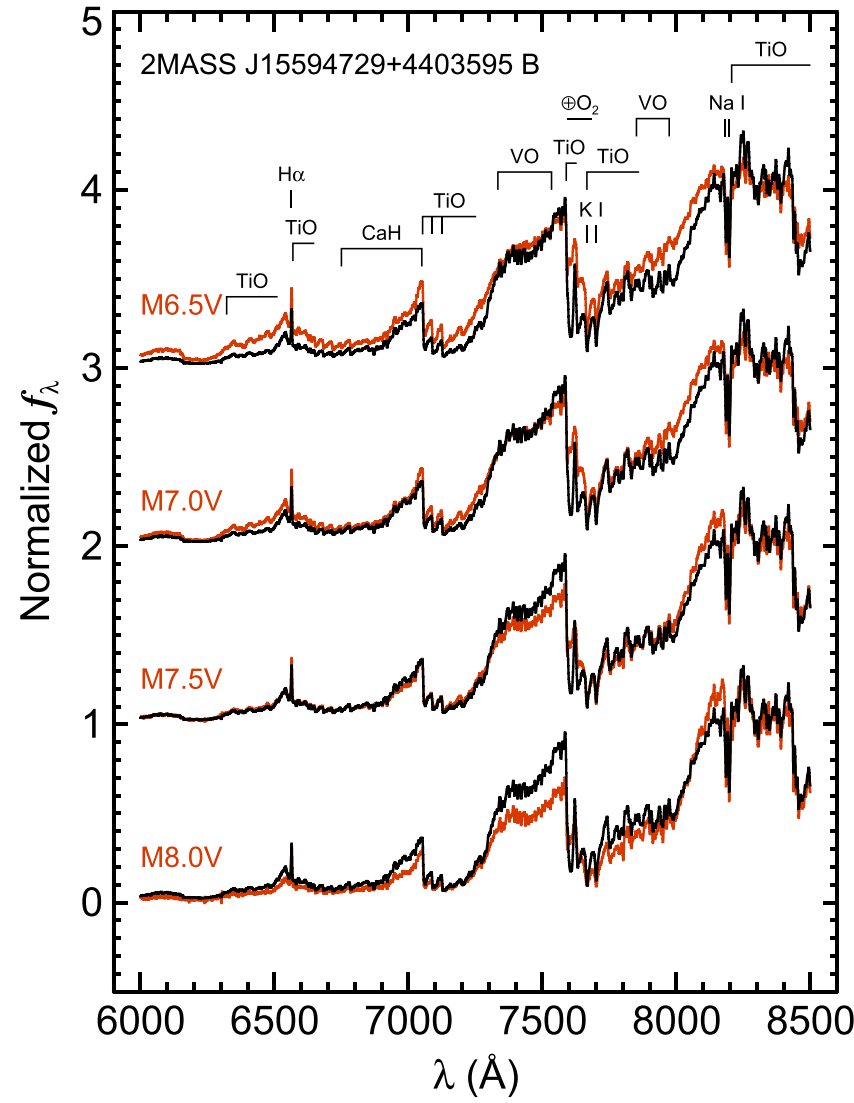

Figure 30. Keck/ESI optical spectrum of 2MASS J15594729 +4403595 B (black). The best matches are M7.0 and M7.5 templates (red) from Bochanski et al. (2007), where the half-types are formed from the average of neighboring integer templates. We adopt an optical spectral type of M7.5 \pm 0.5 but note that the $\approx 7400 \AA$ VO feature appears weaker than the field template. systemic RV of GJ $4378 \mathrm{Aa} 12$ is $21.1 \pm 0.7 \mathrm{~km} \mathrm{~s}^{-1}$. We measure an RV of $20.6 \pm 0.4 \mathrm{~km} \mathrm{~s}^{-1}$ for GJ $4379 \mathrm{~B}$ and find no signs it is a SB. Assuming the RV for GJ $4379 \mathrm{~B}$ represents the systemic velocity, the range of $U V W$ values for distances of $20-60 \mathrm{pc}$ does not coincide with any known moving groups.

Despite their similar spectral types and presumed coevality, GJ $4378 \mathrm{~A}$ and GJ 4379 B appear to have very different chromospheric activity levels. Like PYC J11519+0731 Aab (Section 3.11), GJ 4378 Aa12 is an SB2 with enhanced UV emission (and the likely source of the X-ray emission) probably induced by tidally locked stars instead of intrinsic fast rotation from youth. On the other hand, GJ 4379 B appears to be single and is therefore a better tool to diagnose the system age. Indeed, neither our spectroscopy nor shallow Keck/AO $K_{S}$-band imaging of GJ 4379 B on UT 2014 August 4 shows any signs of a close companion. West et al. (2008) find a characteristic activity lifetime of $4.5_{-1.0}^{+0.5} \mathrm{Gyr}$ for M4 stars as traced by the presence of $\mathrm{H} \alpha$ emission. The lack of $\mathrm{H} \alpha$ emission in GJ 4379 B therefore suggests an old age of $\gtrsim 4$ Gyr for the system.

\subsubsection{GJ $4378 A b$}

We imaged GJ 4378 A in 2012 October with IRCS on Subaru, easily resolving it into a close $\left(0.47^{\prime \prime}\right)$ modest-contrast $(\Delta H=3.1 \mathrm{mag})$ binary. Astrometric monitoring throughout 2013 and 2014 confirm the pair are comoving (Figure 32) and undergoing significant orbital motion of $0.0564^{\prime \prime} \pm$ $0.0006^{\prime \prime} \mathrm{yr}^{-1}$ in separation and $-1.06^{\circ} \pm 0.02^{\circ} \mathrm{yr}^{-1}$ in PA.

In 2013 August we obtained 1-4 $\mu \mathrm{m}$ Keck/NIRC2 AO images of the system (Figure 33). Decomposed photometry of the pair presented in Table 3 implies the companion has red colors consistent with a late-M dwarf. This is confirmed with our $\mathrm{H}$ - and $\mathrm{K}$-band OSIRIS spectroscopy of GJ $4378 \mathrm{Ab}$ (Figure 34), which shows atomic and molecular absorption 


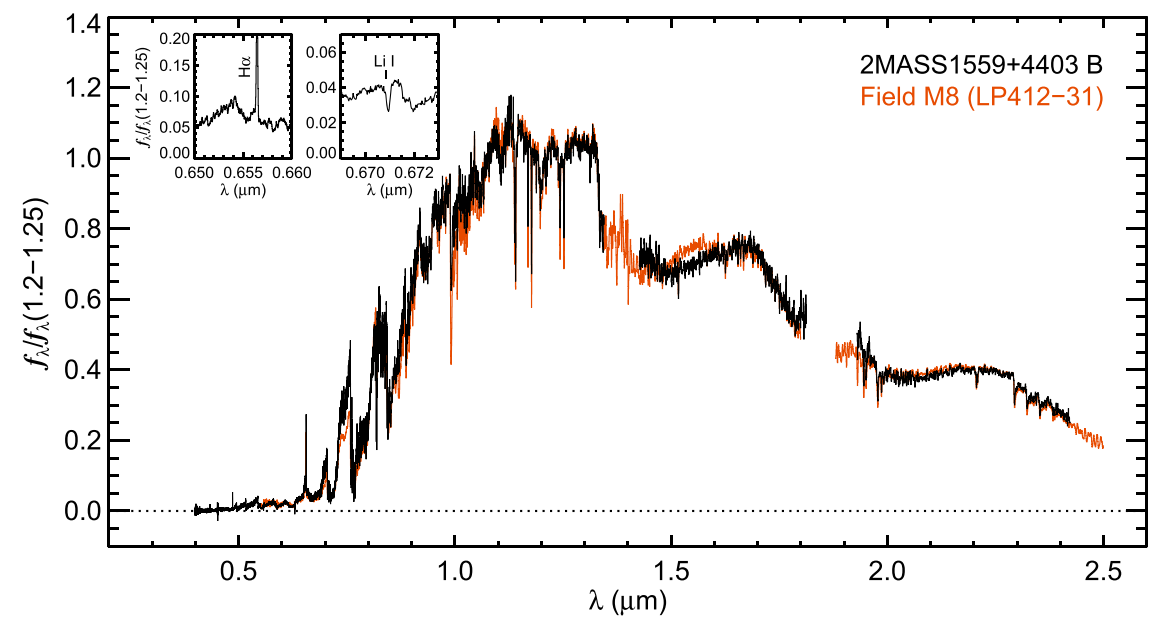

Figure 31. Complete 0.4-2.4 $\mu \mathrm{m}$ spectrum of 2MASS J15594729+4403595 B (black) compared to the high-gravity M8 field object LP $412-31$ (red) from the IRTF Spectral Library. The optical $(0.4-0.9 \mu \mathrm{m})$ region is our new Keck/ESI spectrum, whereas the near-infrared $(0.9-2.4 \mu \mathrm{m})$ region is our IRTF/SpeX spectrum from Bowler et al. (2015). The lithium absorption and angular $H$-band shape in 2MASS J15594729+4403595 B point to an age of $\approx 50-200 \mathrm{Myr}$, making this a nearby young system that does not appear to belong to any known moving groups.
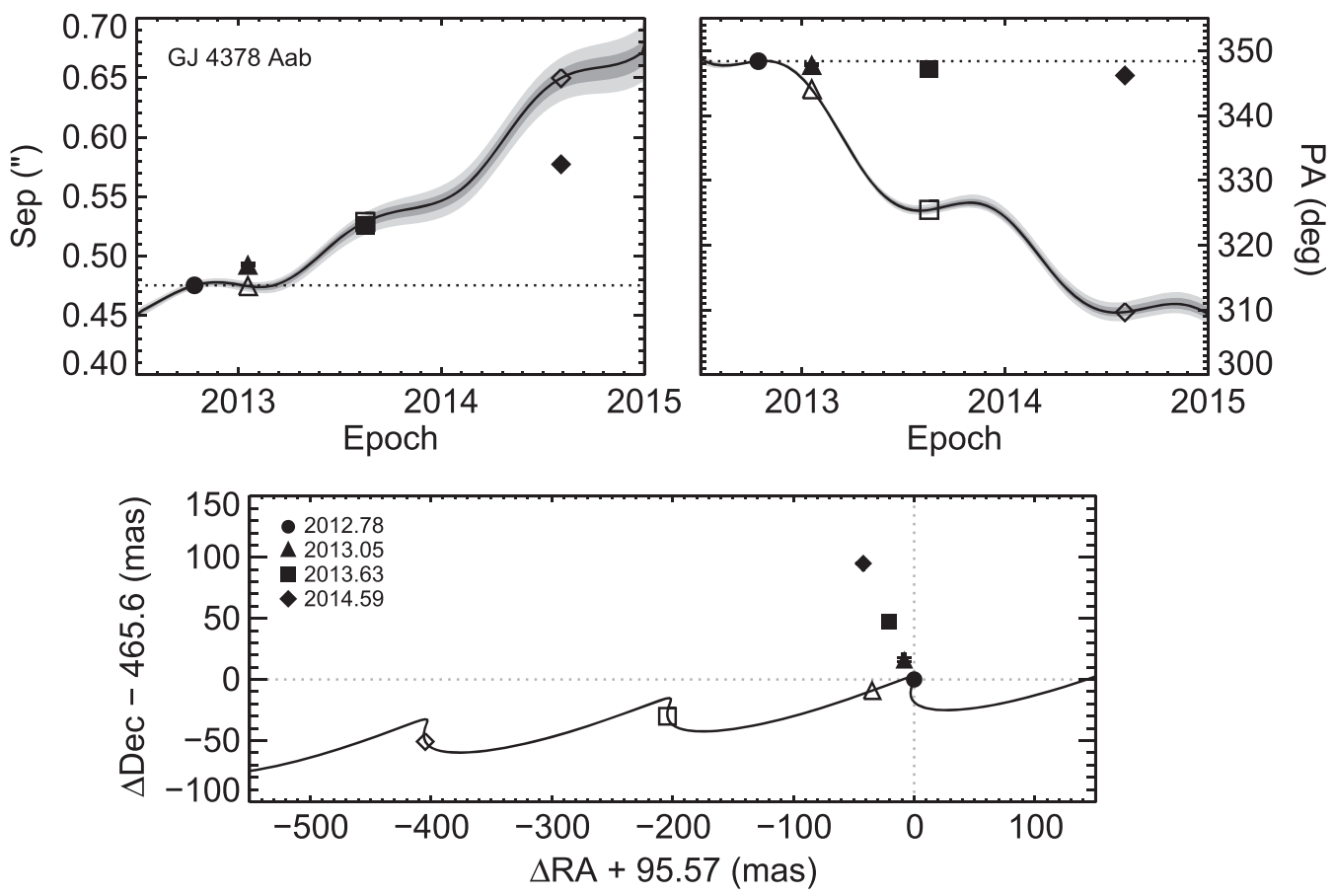

Figure 32. Relative astrometry of GJ 4378 Aa and Ab between 2012 and 2015. The solid curve shows the expected path of a background object, and gray shaded regions show $1 \sigma$ and $2 \sigma$ confidence intervals. The pair are clearly comoving and exhibit significant orbital motion.

features characteristic of late-M/early-L dwarfs. Compared to field late-M dwarfs from Cushing et al. (2005), the overall $1.5-2.4 \mu \mathrm{m}$ spectrum is most similar to the M9V template. The $H$-band spectrum alone provides a reasonable match to the M9V template, whereas the $H$-band spectrum is an excellent match to the M8V template. Altogether we adopt a spectral type of M8 \pm 1 for GJ $4378 \mathrm{Ab}$. Interestingly, the $H$-band spectral shape appears somewhat more angular than the field objects, which would imply a young age $(<150 \mathrm{Myr})$ for the system (Allers \& Liu 2013) and appears to contradict the old age inferred from the lack of $\mathrm{H} \alpha$ emission in the wide companion GJ 4379 B. Moderate-resolution $J$-band spectroscopy of GJ $4378 \mathrm{Ab}$ would help address the system age since the $1.1-1.3 \mu \mathrm{m}$ region possesses a number of gravity-sensitive features.
Using $H$-band spectral-type-absolute magnitude relations from Dupuy \& Liu (2012) and $H$-band bolometric correction from Liu et al. (2010a), we infer a spectrophotometric distance of $14 \pm 2 \mathrm{pc}$ for GJ $4378 \mathrm{Ab}$ and a luminosity of $\log \left(L_{\mathrm{bol}} / L_{*}\right)=-3.35 \pm 0.14$ dex. This agrees with the photometric distance of $12 \mathrm{pc}$ to GJ 4378 Aa from Lépine \& Gaidos (2011). Based on the evolutionary models of Burrows et al. (1997), the luminosity of GJ $4378 \mathrm{Ab}$ implies a mass of $90 \pm 3 M_{\text {Jup }}$ for an age of $5 \pm 2 \mathrm{Gyr}$ with little dependence of age beyond $\approx 1$ Gyr.

\section{DISCUSSION AND CONCLUSIONS}

The high density and sheer number of $\mathrm{M}$ dwarfs in the solar neighborhood imply that many old stars will share similar $U V W$ 

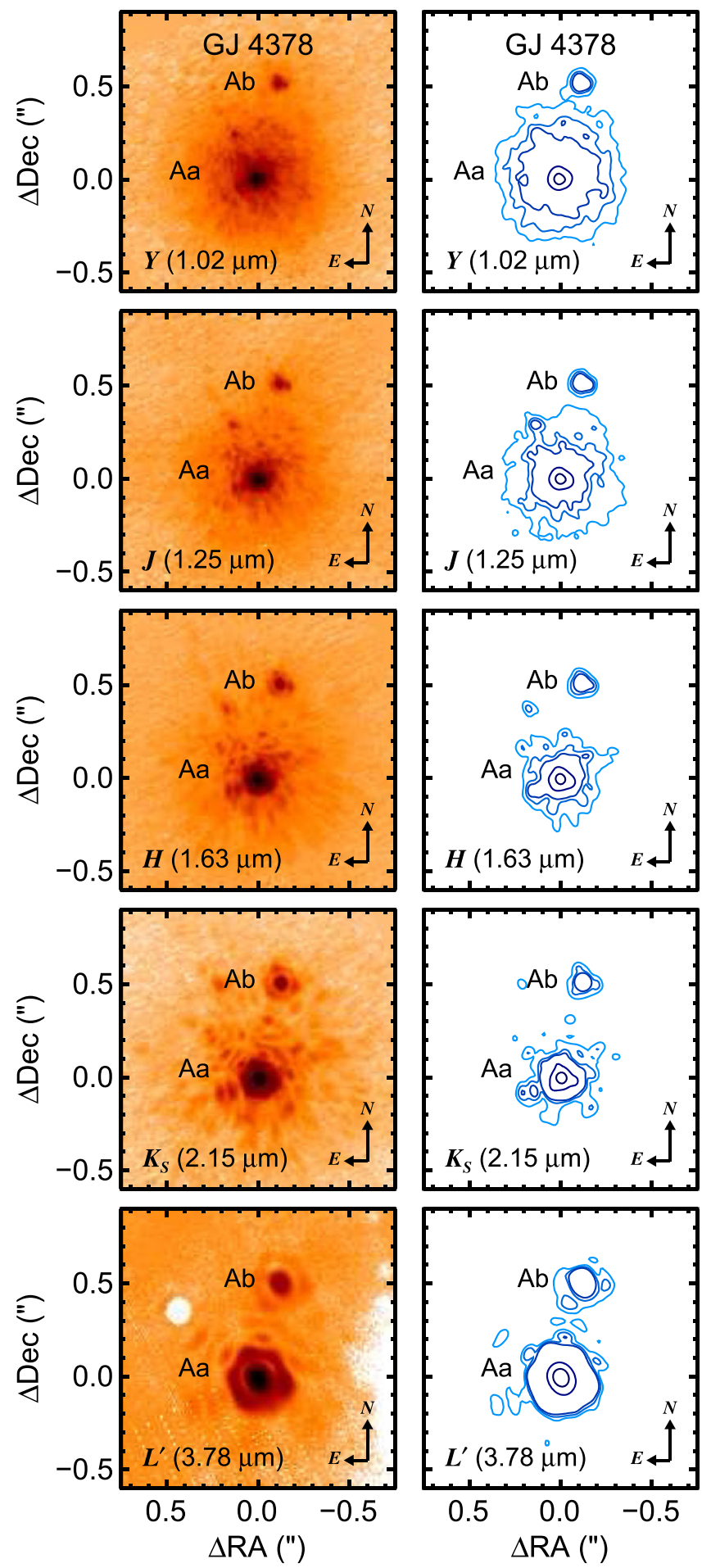

Figure 33. Keck/NIRC2 $Y-, J-, H-, K_{S^{-}}$, and $L^{\prime}$-band $\mathrm{AO}$ images of GJ 4378 Aab. Contours represent $0.3 \%, 0.6 \%, 1 \%, 10 \%$, and $50 \%$ of the peak flux after convolution with a Gaussian kernel with a FWHM equal to that of the image PSF. North is up, and east is left. The field view is $1.5^{\prime \prime} \times 1.5^{\prime \prime}$.

space motions with nearby YMGs. The task of sifting through this haystack of potential members is made even more difficult when only an RV or parallax is available, and in most cases neither of these are. We have undertaken a detailed kinematic and spectroscopic analysis of $13 \mathrm{~K}$ - and M-type systems hosting low-mass companions to clarify their ages, companion masses, and possible associations with YMGs (Figure 35). Eleven of the primary stars have been previously identified as being young in the literature, eight of which have been listed as candidate members of YMGs. Three of the late-type companions to moving group candidates were discovered during our PALMS AO imaging survey and are new in this work (2MASS J06475229-2523304 B, PYC J11519+0731 B, and GJ $4378 \mathrm{Ab})$.

Only two of the systems appear to be likely members of YMGs (2MASS J01225093-2439505 AB and 2MASS J02155892-0929121 AabBC). Another four show signs of youth but are probably not kinematically associated with any known moving groups (1RXS J034231.8+121622 AB, 2MASS J08540240-3051366 AB, G 180-11 AB, and 2MASS $\mathrm{J} 15594729+4403595 \mathrm{AB})$. With the exception of 2MASS $\mathrm{J} 11240434+3808108 \mathrm{~B}$, whose kinematics are consistent with the $~ 500$ Myr Ursa Major moving group, the remaining systems with low-mass companions are probably old. In particular, we have shown that the host stars to 2MASS J06475229-2523304 B, PYC J11519+0731 B, and GJ 4378 Ab are close SBs with little or no evidence that they belong to nearby YMGs. This underscores the need for follow-up RV (and ideally parallax) measurements for new proposed members of YMGs to identify old M + M SB1s and SB2s that can masquerade as young stars.

2MASS J02155892-0929121 AabBC is likely to be an especially useful system in the future. If its systemic RV and parallax confirm it is a member of Tuc-Hor, which is suggested by the mean $\left(4.0 \pm 0.15 \mathrm{~km} \mathrm{~s}^{-1}\right)$ and range $\left(-0.6-10.0 \mathrm{~km} \mathrm{~s}^{-1}\right)$ of RV measurements, 2MASS J02155892-0929121 will offer an excellent opportunity to measure dynamical masses for the Aab components and, eventually, the B component. Because the outer companion ("C") is a young brown dwarf, this quadruple system offers the opportunity to test pre-mainsequence evolutionary models across the substellar boundary. Regardless of any possible age spread in Tuc-Hor itself, the components of this system were probably formed simultaneously, removing any ambiguity about possible age differences. This is analogous to the well-studied young high-order benchmarks LkCa 3 (Torres et al. 2013), HD 98800 (e.g., Prato et al. 2001), and especially GG Tau (White et al. 1999), which also contains a substellar member and recently gained a fifth component from sparse aperture masking interferometry (Di Folco et al. 2014).

Four systems in our sample are examples of close SBs on what are likely tidally circularized, few-day orbits with nearby lower-mass tertiaries that we have directly resolved with AO (G 160-54 AabBC, 2MASS J06475229-2523304 AabB, PYC J11519+0731 AabB, and GJ 4378 Aa12b). SBs do not form in situ but instead must lose angular momentum either through gas drag in a viscous molecular cloud core during the protostellar phase (e.g., Gorti \& Bhatt 1996; Stahler 2010) or through dynamical interactions with a third body (e.g., Eggleton \& Kiseleva-Eggleton 2001). These close hierarchical triples are consistent with this latter scenario, in which the tertiary remained relatively close in after a dynamical encounter with the (now tidally locked) secondary star instead of being ejected to a wide orbit or completely out of the system (Tokovinin et al. 2006; Reipurth et al. 2010; Allen et al. 2012; Reipurth \& Mikkola 2013). These tertiaries are expected to have relatively high eccentricities, which can eventually be tested with continued astrometric monitoring.

Regardless of their ages, many of these systems are useful for calibrating empirical metallicity relationships at the bottom 

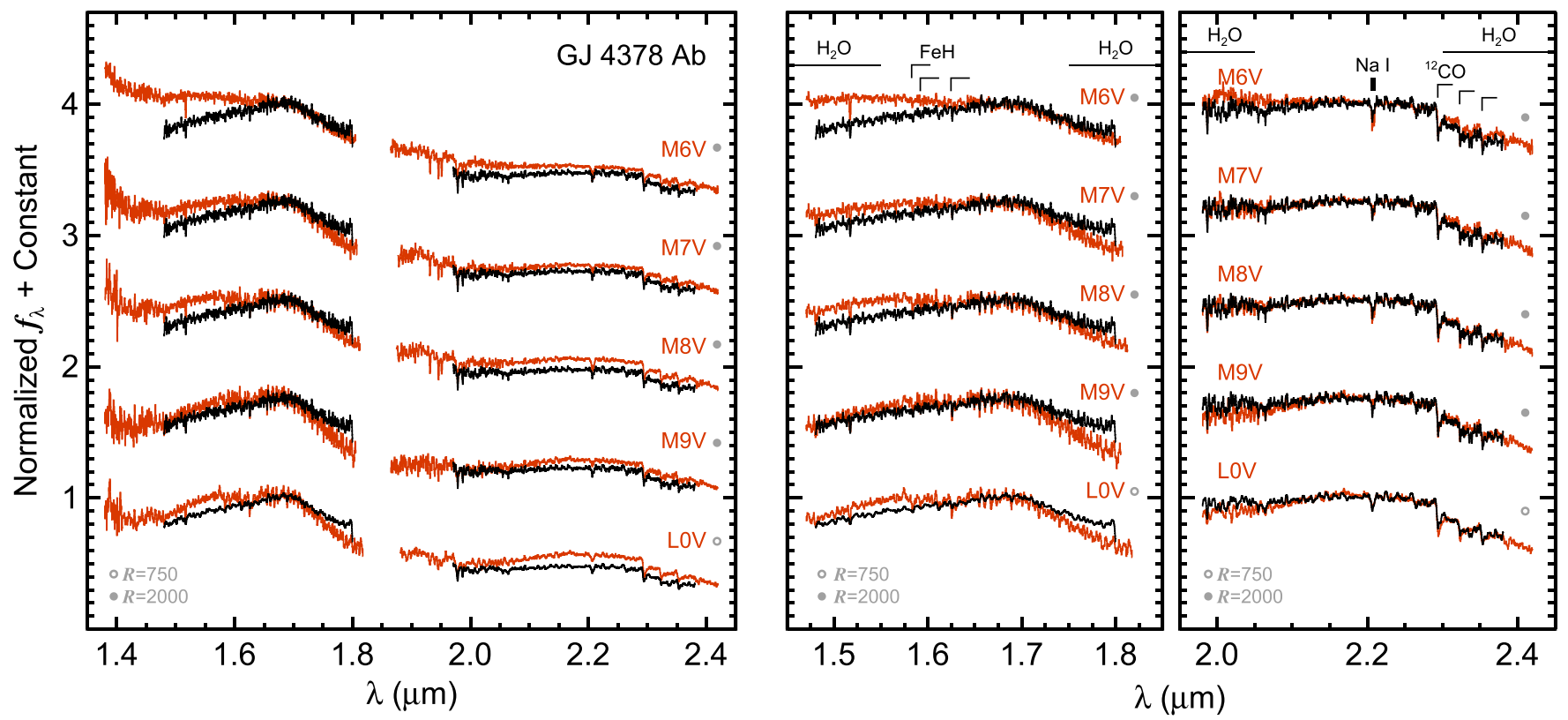

Figure 34. Keck/OSIRIS 1.5-2.4 $\mu \mathrm{m}$ spectrum of GJ $4378 \mathrm{Ab}$ (black). The best match is to M8-M9 field templates (red) from the IRTF Spectral Library. We adopt a near-infrared spectral type of M8 \pm 1 . Note that the slightly angular $H$-band shape, which points to a young age of $\lesssim 150 \mathrm{Myr}$, is at odds with the lack of $\mathrm{H} \alpha$ emission in the wide comoving companion GJ $4379 \mathrm{~B}$, which suggests an old age of several Gyr. Our OSIRIS spectrum has been Gaussian-smoothed to match the resolving power of the templates $(R \approx 750-2000)$, and all spectra are normalized and offset by a constant.

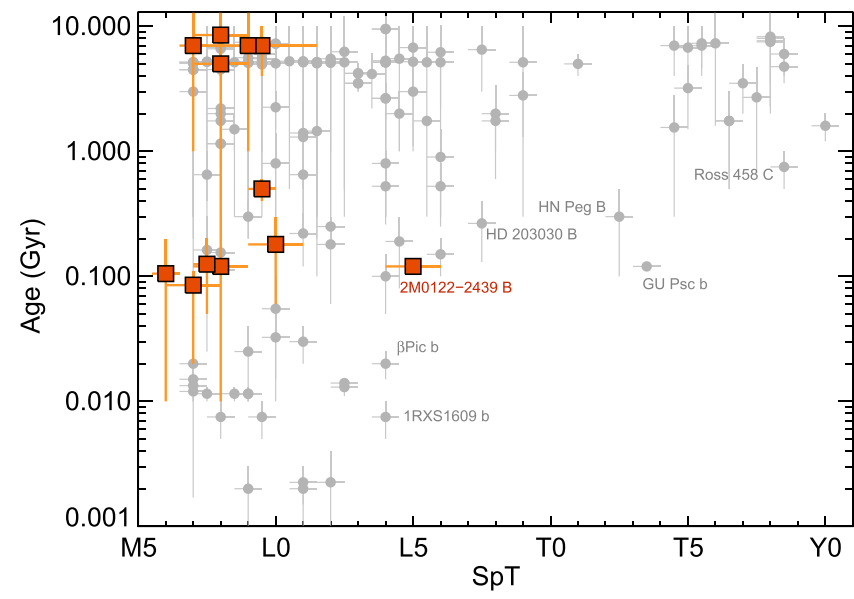

Figure 35. The known ultracool companions to stars. Systems analyzed in this work are denoted with red squares and mostly have late-M spectral types. The dearth of young ( $\lesssim 100 \mathrm{Myr})$ companions later than $\sim \mathrm{L} 5$ reflects the paucity of young planets discovered via direct imaging. Note that we have excluded the HR 8799 planets (Marois et al. 2008, 2010), GJ 504 b (Kuzuhara et al. 2013), and HD 95086 b (Rameau et al. 2013) because their spectral types are either poorly constrained or may defy conventional classification schemes. Known companions (gray circles) are from Deacon et al. (2014) and are supplemented with our own compilation.

of the main sequence. Recently, Mann et al. (2014) presented an empirical method to measure the metallicities of late-M dwarfs with $K$-band spectra by leveraging the metallicity of wide, earlier-type binary companions for calibration. Although this was the largest such study to date, only a handful of late-M dwarfs in binaries were used (seven systems beyond M7.5), so building larger samples of these benchmark systems will help better constrain these relationships.

Gaia will soon provide precise parallaxes for the currently unknown stellar members of YMGs, but its estimated $1-15 \mathrm{~km} \mathrm{~s}^{-1} \mathrm{RV}$ precision is generally not adequate to differentiate moving groups with $U V W$ space velocities only a few $\mathrm{km} \mathrm{s}^{-1}$ apart like TWA, Tuc-Hor, and Carina (Lindegren et al. 2008; Eyer et al. 2015). Ground-based RVs with $<1 \mathrm{~km} \mathrm{~s}^{-1}$ precisions will therefore continue to play a critical role in confirming this nearby population of young stars in the foreseeable future.

We thank the referee for their helpful suggestions and Niall Deacon for obtaining some of the IRTF observations presented here. M.C.L. has been supported by NASA Grant NNX11AC31G and NSF Grant AST09-09222. This paper is based on observations at Cerro Tololo Inter-American Observatory, National Optical Astronomy Observatory (NOAO Prop. ID: 2014B-0083; PI: Bowler), which is operated by the Association of Universities for Research in Astronomy (AURA) under a cooperative agreement with the National Science Foundation. This research is also based in part on observations obtained at the Gemini Observatory, which is operated by AURA under a cooperative agreement with the NSF on behalf of the Gemini partnership: the National Science Foundation (United States), the Science and Technology Facilities Council (United Kingdom), the National Research Council (Canada), CONICYT (Chile), the Australian Research Council (Australia), CNPq (Brazil) and CONICET (Argentina). The Robo-AO system was developed by collaborating partner institutions, the California Institute of Technology and the Inter-University Centre for Astronomy and Astrophysics, and with the support of the National Science Foundation under Grants AST-0906060, AST-0960343, and AST-1207891, the Mt. Cuba Astronomical Foundation, and by a gift from Samuel Oschin. Ongoing science operation support of Robo-AO is provided by the California Institute of Technology and the University of Hawai'i. C.B. acknowledges support from the Alfred P. Sloan Foundation. B.T.M. is supported by a National Science Foundation Graduate Research Fellowship under Grant DGE1144469. We utilized data products from the Two 
Micron All Sky Survey, which is a joint project of the University of Massachusetts and the Infrared Processing and Analysis Center/California Institute of Technology, funded by the National Aeronautics and Space Administration and the National Science Foundation. NASA's Astrophysics Data System Bibliographic Services together with the VizieR catalog access tool and SIMBAD database operated at CDS, Strasbourg, France, were invaluable resources for this work. This research has made use of the Washington Double Star Catalog maintained at the U.S. Naval Observatory. Finally, mahalo nui loa to the kama'âina of Hawai'i for their support of Keck and the Maunakea observatories. We are grateful to conduct observations from this mountain.

Facilities: Keck:II (NIRC2, ESI), Subaru (IRCS), Gemini: South (NICI), PO: $1.5 \mathrm{~m}$ (Robo-AO), Keck:I (OSIRIS, HIRES), IRTF (SpeX), Mayall (RC-Spec), SOAR (Goodman), UH:2.2 m (SNIFS), CFHT (ESPaDOnS), Du Pont (Echelle), Max Planck:2.2 m (FEROS), CTIO:1.5 m (CHIRON).

Note Added in Proof. Recently we were notified that the All Sky Automated Survey light curve of 2MASS J064752292523304 shows particularly large amplitude variations of 0.33 mag and has structure resembling an eclipsing binary with a period of 7.561 days (or alias thereof). The K7 spectral type we found is cooler than the standard subgiant branch, so this may be an example of a poorly understood but relatively nearby "sub-subgiant" or "red straggler" system, a class of objects that occupy an unusual position in the color-magnitude diagram and have primarily been found in open and globular clusters. We thank E. Mamajek and A. Kraus for bringing this to our attention.

\section{REFERENCES}

Ahn, C. P., Alexandroff, R., Allende Prieto, C., et al. 2012, ApJS, 203, 21 Aldering, G., Antilogus, P., Bailey, S., et al. 2006, ApJ, 650, 510

Allen, P. R., Burgasser, A. J., Faherty, J. K., \& Kirkpatrick, J. D. 2012, AJ, 144,62

Allers, K. N., Jaffe, D. T., Luhman, K. L., et al. 2007, ApJ, 657, 511

Allers, K. N., \& Liu, M. C. 2013, ApJ, 772, 79

Ammons, S. M., Robinson, S. E., Strader, J., et al. 2006, ApJ, 638, 1004

Baraffe, I., Chabrier, G., Allard, F., \& Hauschildt, P. H. 1998, A\&A, 337, 403

Baraffe, I., Chabrier, G., Barman, T. S., Allard, F., \& Hauschildt, P. H. 2003, A\&A, 402, 701

Baranec, C., Riddle, R., Law, N. M., et al. 2014, ApJL, 790, L8

Baranec, C., Riddle, R., Law, N. M., et al. 2013, JoVE, 72, 1

Barman, T. S., Macintosh, B., Konopacky, Q. M., \& Marois, C. 2011, ApJ, 733,65

Basri, G. 1998, in ASP Conf. Ser 134, Brown Dwarfs and Extrasolar Planets, ed. R. Rebolo, E. L. Martin, \& M. R. Zapatero Osorio (San Francisco, CA: ASP), 394

Bender, C. F., \& Simon, M. 2008, ApJ, 689, 416

Bergfors, C., Brandner, W., Janson, M., et al. 2010, A\&A, 520, A54

Bessell, M. S., \& Brett, J. M. 1988, PASP, 100, 1134

Biller, B. A., Liu, M. C., Wahhaj, Z., et al. 2010, ApJL, 720, L82

Binks, A. S., \& Jeffries, R. D. 2014, MNRAS Letters, 438, L11

Bochanski, J. J., Hennawi, J. F., Simcoe, R. A., et al. 2009, PASP, 121, 1409

Bochanski, J. J., West, A. A., Hawley, S. L., \& Covey, K. R. 2007, AJ, 133,531

Bowler, B. P., Liu, M. C., Dupuy, T. J., \& Cushing, M. C. 2010, ApJ, 723, 850 Bowler, B. P., Liu, M. C., Kraus, A. L., \& Mann, A. W. 2014, ApJ, 784, 65 Bowler, B. P., Liu, M. C., Shkolnik, E. L., \& Dupuy, T. J. 2013, ApJ, 774, 55 Bowler, B. P., Liu, M. C., Shkolnik, E. L., et al. 2012a, ApJ, 753, 142 Bowler, B. P., Liu, M. C., Shkolnik, E. L., \& Tamura, M. 2012b, ApJ, 756, 69 Bowler, B. P., Liu, M. C., Shkolnik, E. L., \& Tamura, M. 2015, ApJS, 216, 7 Boyajian, T. S., von Braun, K., van Belle, G., et al. 2012, ApJ, 757, 112 Brewer, J. M., Giguere, M., \& Fischer, D. A. 2014, PASP, 126, 48 Burrows, A., Marley, M., Hubbard, W. B., et al. 1997, ApJ, 491, 856
Buton, C., Copin, Y., Aldering, G., et al. 2012, A\&A, 549, A8 Casagrande, L., Flynn, C., \& Bessell, M. 2008, MNRAS, 389, 585 Cenko, S. B., Fox, D. B., Moon, D. S., et al. 2006, PASP, 118, 1396 Chabrier, G., Baraffe, I., \& Plez, B. 1996, ApJL, 459, L91

Chauvin, G., Lagrange, A.-M., Zuckerman, B., et al. 2005, A\&A, 438, L29 Chauvin, G., Lagrange, A.-M., Bonavita, M., et al. 2010, A\&A, 509, A52 Chubak, C., Marcy, G., Fischer, D. A., et al. 2012, arXiv:1207.6212 Chun, M., Toomey, D., Wahhaj, Z., et al. 2008, Proc. SPIE, 7015, 70151

Close, L. M., Siegler, N., Freed, M., \& Biller, B. 2003, ApJ, 587, 407

Covey, K. R., Ivezić, Ž., Schlegel, D., et al. 2007, AJ, 134, 2398

Crepp, J. R., Johnson, J. A., Howard, A. W., et al. 2014, ApJ, 781, 29

Cruz, K. L., Reid, I. N., Liebert, J., Kirkpatrick, J. D., \& Lowrance, P. J. 2003 , AJ, 126, 2421

Cushing, M. C., Rayner, J. T., \& Vacca, W. D. 2005, ApJ, 623, 1115

Cushing, M. C., Vacca, W. D., \& Rayner, J. T. 2004, PASP, 116, 362

Daemgen, S., Siegler, N., Reid, I. N., \& Close, L. M. 2007, ApJ, 654, 558

Deacon, N. R., Liu, M. C., Magnier, E. A., et al. 2014, ApJ, 792, 119

Delorme, P., Gagné, J., Malo, L., et al. 2012, A\&A, 548, A26

Delorme, P., Gagné, J., Girard, J. H., et al. 2013, A\&A, 553, L5

di Folco, E., Dutrey, A., le Bouquin, J.-B., et al. 2014, A\&A, 565, L2

Dittmann, J. A., Irwin, J. M., Charbonneau, D., \& Berta-Thompson, Z. K. 2014, ApJ, 784, 156

do Nascimento, J. D., Jr., da Costa, J. S., \& Castro, M. 2012, A\&A, 548, L1

Donati, J.-F., Catala, C., Landstreet, J. D., \& Petit, P. 2006, in ASP Conf. Ser. 358, Solar Polarization 4, ed. R. Casini, \& B. W. Lites (San Francisco, CA: ASP), 362

Donati, J. F., Semel, M., Carter, B. D., Rees, D. E., \& Collier-Cameron, A. 1997, MNRAS, 291, 658

Drilling, J. S., \& Landolt, A. U. 2000, in Allen's Astrophysical Quantities ed. A. N. Cox (4th ed.; New York: AIP; Springer), 381

Droege, T. F., Richmond, M. W., Sallman, M. P., \& Creager, R. P. 2006, PASP, 118, 1666

Dupuy, T. J., \& Liu, M. C. 2012, ApJS, 201, 19

Dupuy, T. J., Liu, M. C., \& Ireland, M. J. 2014, ApJ, 790, 133

Eggleton, P. P., \& Kiseleva-Eggleton, L. 2001, ApJ, 562, 1012

Eyer, L., Rimoldini, L., Holl, B., et al. 2015, arXiv:1502.03829

Faherty, J. K., Rice, E. L., Cruz, K. L., Mamajek, E. E., \& Núñez, A. 2012, AJ, 145,2

Filippenko, A. V. 1982, Astronomical Society of the Pacific, 94, 715

Fitzpatrick, M. J. 1993, in ASP Conf. Ser. 52, Astronomical Data Analysis Software and Systems II, ed. R. J. Hanish, R. V. J. Brissenden, \& J. Barnes (San Francisco, CA: ASP), 472

Foreman-Mackey, D., Hogg, D. W., Lang, D., \& Goodman, J. 2013, PASP, 125,306

Gagné, J., LaFreniere, D., Doyon, R., Malo, L., \& Artigau, E. 2014, ApJ, 783,121

Giclas, H. L., Burnham, R. J., \& Thomas, N. G. 1975, LowOB, 8, 9

Gizis, J. E., Jao, W.-C., Subasavage, J. P., \& Henry, T. J. 2007, ApJL, 669, L45 Golimowski, D. A., Leggett, S. K., Marley, M. S., et al. 2004, AJ, 127, 3516 Goodman, J., \& Weare, J. 2010, Communications in Applied Mathematics and Computational Science, 5, 65

Gorti, U., \& Bhatt, H. C. 1996, MNRAS, 283, 566

Gray, R. O., Napier, M. G., \& Winkler, L. I. 2001, AJ, 121, 2148

Haakonsen, C. B., \& Rutledge, R. E. 2009, ApJS, 184, 138

Hamuy, M., Suntzeff, N. B., Heathcote, S. R., et al. 1994, Astronomical Society of the Pacific, 106, 566

Hamuy, M., Walker, A. R., Suntzeff, N. B., et al. 1992, Astronomical Society of the Pacific, 104, 533

Hartman, J. D., Bakos, G. Á, Noyes, R. W., et al. 2011, AJ, 141, 166

Hawley, S. L., Gizis, J. E., \& Reid, I. N. 1996, AJ, 112, 2799

Hayano, Y., Takami, H., Oya, S., et al. 2010, Proc. SPIE, 7736, 77360

Hayward, T. L., Biller, B. A., Liu, M. C., et al. 2014, PASP, 126, 1112

Hillenbrand, L. A., Foster, J. B., Persson, S. E., \& Matthews, K. 2002, PASP, 114,708

Horne, K. 1986, Astronomical Society of the Pacific, 98, 609

Janson, M., Hormuth, F., Bergfors, C., et al. 2012, ApJ, 754, 44

Janson, M., Bergfors, C., Brandner, W., et al. 2014, ApJS, 214, 17

Johnson, D. R. H., \& Soderblom, D. R. 1987, AJ, 93, 864

Kaufer, A., Stahl, O., Tubbesing, S., et al. 1999, Msngr, 95, 8

Kiraga, M. 2012, AcA, 62, 67

Kirkpatrick, J. D., Cruz, K. L., Barman, T. S., et al. 2008, ApJ, 689, 1295

Kobayashi, N., Tokunaga, A. T., Terada, H., et al. 2000, Proc. SPIE, 4008, 1056

Kordopatis, G., Gilmore, G., Steinmetz, M., et al. 2013, AJ, 146, 134

Kraus, A. L., Ireland, M. J., cieza, L. A., et al. 2014a, ApJ, 781, 20

Kraus, A. L., Shkolnik, E. L., Allers, K. N., \& Liu, M. C. 2014b, AJ, 147, 146 
Kraus, A. L., Tucker, R. A., Thompson, M. I., Craine, E. R., \& Hillenbrand, L. A. 2011, ApJ, 728, 48

Kuzuhara, M., Tamura, M., Kudo, T., et al. 2013, ApJ, 774, 11

Lafrenière, D., Marois, C., Doyon, R., Nadeau, D., \& Artigau, É 2007, ApJ, 660,770

Lantz, B., Aldering, G., Antilogus, P., et al. 2004, Proc. SPIE, 5249, 146

Larkin, J., Barczys, M., Krabbe, A., et al. 2006, Proc. SPIE, 6269, 42

Law, N. M., Hodgkin, S. T., \& Mackay, C. D. 2008, MNRAS, 384, 150

Law, N. M., Morton, T., Baranec, C., et al. 2014, ApJ, 791, 35

Leggett, S. K., Currie, M. J., Varricatt, W. P., et al. 2006, MNRAS, 373, 781

Lépine, S., \& Gaidos, E. 2011, AJ, 142, 138

Lépine, S., Hilton, E. J., Mann, A. W., et al. 2013, AJ, 145, 102

Lindegren, L., Babusiaux, C., Bailer-Jones, C., et al. 2008, A Giant Step: from Milli- to Micro-arcsecond Astrometry (IAU Symp. 217) ed. W. Jin, I. Platais, \& M. A. C. Perryman (Cambridge: Cambridge Univ. Press)

Liu, M. C. 2004, Sci, 305, 1442

Liu, M. C., Dupuy, T. J., \& Allers, K. N. 2013a, AN, 334, 85

Liu, M. C., Dupuy, T. J., Bowler, B. P., Leggett, S. K., \& Best, W. M. J. 2012 ApJ, 758, 57

Liu, M. C., Dupuy, T. J., \& Ireland, M. J. 2008, ApJ, 689, 436

Liu, M. C., Dupuy, T. J., \& Leggett, S. K. 2010a, ApJ, 722, 311

Liu, M. C., Magnier, E. A., Deacon, N. R., et al. 2013b, ApJL, 777, L20

Liu, M. C., Wahhaj, Z., Biller, B. A., et al. 2010b, in Proc. SPIE, 7736, 77361

Looper, D. L., Bochanski, J. J., Burgasser, A. J., et al. 2010, AJ, 140, 1486

Looper, D. L., Burgasser, A. J., Kirkpatrick, J. D., \& Swift, B. J. 2007, ApJL, 669, L97

Lowrance, P. J., McCarthy, C., Becklin, E. E., et al. 1999, ApJL, 512, L69

Lowrance, P. J., Schneider, G., Kirkpatrick, J. D., et al. 2000, ApJ, 541, 390

Malo, L., Artigau, E., Doyon, R., et al. 2014, ApJ, 788, 81

Malo, L., Doyon, R., Lafrenière, D., et al. 2013, ApJ, 762, 88

Mamajek, E. E., \& Bell, C. P. M. 2014, MNRAS, 445, 2169

Mann, A. W., Deacon, N. R., Gaidos, E., et al. 2014, AJ, 147, 160

Mann, A. W., Gaidos, E., \& Ansdell, M. 2013, ApJ, 779, 188

Mann, A. W., Gaidos, E., Lépine, S., \& Hilton, E. J. 2012, ApJ, 753, 90

Marley, M. S., Saumon, D., Cushing, M., et al. 2012, ApJ, 754, 135

Marois, C., Lafrenière, D., Doyon, R., Macintosh, B., \& Nadeau, D. 2006, ApJ, 641, 556

Marois, C., Macintosh, B., Barman, T., et al. 2008, Sci, 322, 1348

Marois, C., Zuckerman, B., Konopacky, Q. M., Macintosh, B., \& Barman, T. 2010, Natur, 468, 1080

Mazeh, T., Prato, L., Simon, M., et al. 2002, ApJ, 564, 1007

McCarthy, C., Zuckerman, B., \& Becklin, E. E. 2001, AJ, 121, 3259

McGovern, M. R., Kirkpatrick, J. D., McLean, I. S., et al. 2004, ApJ, 600, 1020

Melis, C., Reid, M. J., Mioduszewski, A. J., Stauffer, J. R., \& Bower, G. C. 2014, Sci, 345, 1029

Mentuch, E., Brandeker, A., van Kerkwijk, M. H., Jayawardhana, R., \& Hauschildt, P. H. 2008, ApJ, 689, 1127

Mieda, E., Wright, S. A., Larkin, J. E., et al. 2014, PASP, 126, 250

Monet, D. G., Levine, S. E., Canzian, B., et al. 2003, AJ, 125, 984

Mugrauer, M., Vogt, N., Neuhäuser, R., \& Schmidt, T. O. B. 2010, A\&A, 523, L1

Muiños, J. L., \& Evans, D. W. 2014, AN, 335, 367

Müller, A., Roccatagliata, V., Henning, T., et al. 2013, A\&A, 556, A3

Murray, C. A. 1989, A\&A, 218, 325

Naud, M.-E., Artigau, E., Malo, L., et al. 2014, ApJ, 787, 5

Nelder, J. A., \& Mead, R. 1965, CompJ, 7, 308

Nidever, D. L., Marcy, G. W., Butler, R. P., Fischer, D. A., \& Vogt, S. S. 2002, ApJS, 141, 503

Pecaut, M. J., \& Mamajek, E. E. 2013, ApJS, 208, 9

Pickles, A., \& Depagne, É 2010, PASP, 122, 1437

Pojmanski, G. 1997, AcA, 47, 467

Prato, L., Ghez, A. M., Piña, R. K., et al. 2001, ApJ, 549, 590

Prato, L., Simon, M., Mazeh, T., et al. 2002, ApJ, 569, 863

Press, W., Teukolsky, S. A., Vetterling, W. T., \& Flannery, B. P. 2007, in Numerical Recipes: The Art of Scientific Computing (3rd ed.)

Prochaska, J. X., Gawiser, E., Wolfe, A. M., Cooke, J., \& Gelino, D. 2003, ApJS, 147, 227

Rameau, J., Chauvin, G., Lagrange, A.-M., et al. 2013, ApJL, 779, L26
Rayner, J. T., Cushing, M. C., \& Vacca, W. D. 2009, ApJS, 185, 289

Rebolo, R., Magazzù, A., \& Martin, E. L. 1995, MmSAI, 66, 375

Rebolo, R., Martin, E. L., Basri, G., Marcy, G. W., \& Zapatero-Osorio, M. R. 1996, ApJL, 469, L53

Rebolo, R., Martin, E. L., \& Magazzu, A. 1992, ApJL, 389, L83

Reid, I. N., Cruz, K. L., Allen, P., et al. 2004, AJ, 128, 463

Reid, I. N., Cruz, K. L., \& Allen, P. R. 2007, AJ, 133, 2825

Reid, I. N., Hawley, S. L., \& Gizis, J. E. 1995, AJ, 110, 1838

Reiners, A., \& Basri, G. 2009, ApJ, 705, 1416

Reipurth, B., \& Mikkola, S. 2013, Natur, 492, 221

Reipurth, B., Mikkola, S., Connelley, M., \& Valtonen, M. 2010, ApJL, 725, L56

Rhee, J. H., Song, I., \& Zuckerman, B. 2008, ApJ, 675, 777

Riaz, B., Gizis, J. E., \& Harvin, J. 2006, AJ, 132, 866

Richards, J. W., Starr, D. L., Miller, A. A., et al. 2012, ApJS, 203, 32

Riedel, A. R., Finch, C. T., Henry, T. J., et al. 2014, AJ, 147, 85

Rodriguez, D. R., Marois, C., Zuckerman, B., Macintosh, B., \& Melis, C. 2012, ApJ, 748, 30

Rodriguez, D. R., Zuckerman, B., Kastner, J. H., et al. 2013, ApJ, 774, 101

Roeser, S., Demleitner, M., \& Schilbach, E. 2010, AJ, 139, 2440

Röser, S., Schilbach, E., Schwan, H., et al. 2008, A\&A, 488, 401

Ryan, S. G., \& Deliyannis, C. P. 1995, ApJ, 453, 819

Scalzo, R. A., Aldering, G., Antilogus, P., et al. 2010, ApJ, 713, 1073

Schlieder, J. E., Lépine, S., Rice, E., et al. 2012a, AJ, 143, 114

Schlieder, J. E., Lépine, S., \& Simon, M. 2012b, AJ, 143, 80

Scholz, R.-D., Mccaughrean, M. J., Zinnecker, H., \& Lodieu, N. 2005, A\&A, 430, L49

Sheinis, A. I., Bolte, M., Epps, H. W., et al. 2002, PASP, 114, 851

Shkolnik, E., Liu, M. C., \& Reid, I. N. 2009, ApJ, 699, 649

Shkolnik, E. L., Anglada-Escudé, G., Liu, M. C., et al. 2012, ApJ, 758, 56

Shkolnik, E. L., Hebb, L., Liu, M. C., Reid, I. N., \& Cameron, A. C. 2010, ApJ, 716, 1522

Simons, D. A., \& Tokunaga, A. 2002, PASP, 114, 169

Skemer, A. J., Marley, M. S., Hinz, P. M., et al. 2014, ApJ, 792, 17

Skrutskie, M. F., Cutri, R. M., Stiening, R., et al. 2006, AJ, 131, 1163

Slesnick, C. L., Hillenbrand, L. A., \& Carpenter, J. M. 2004, ApJ, 610 1045

Stahler, S. W. 2010, MNRAS, 402, 1758

Stauffer, J. R., Schultz, G., \& Kirkpatrick, J. D. 1998, ApJL, 499, L199

Taylor, B. J. 2008, AJ, 136, 1388

Tokovinin, A., Fischer, D. A., Bonati, M., et al. 2013, PASP, 125, 1336

Tokovinin, A., Thomas, S., Sterzik, M., \& Udry, S. 2006, A\&A, 450, 681

Tokunaga, A. T., Kobayashi, N., Bell, J., et al. 1998, Proc. SPIE, 3354, 512

Tokunaga, A. T., \& Vacca, W. D. 2005, PASP, 117, 421

Torres, C. A. O., Quast, G. R., da Silva, L., et al. 2006, A\&A, 460, 695

Torres, C. A. O., Quast, G. R., Melo, C. H. F., \& Sterzik, M. F. 2008, in Young Nearby Loose Associations, ed. B. Reipurth (San Francisco, CA: ASP), 757

Torres, G., Guenther, E. W., Marschall, L. A., et al. 2003, AJ, 125, 825

Torres, G., Neuhäuser, R., \& Guenther, E. W. 2002, AJ, 123, 1701

Torres, G., Ruiz-Rodriguez, D., Badenas, M., et al. 2013, ApJ, 773, 40

Vacca, W. D., Cushing, M. C., \& Rayner, J. T. 2003, PASP, 115, 389

van Saders, J. L., \& Pinsonneault, M. H. 2013, ApJ, 776, 67

Voges, W., Aschenbach, B., Boller, T., et al. 1999, A\&A, 349, 389

Vogt, S. S., Allen, S. L., Bigelow, B. C., et al. 1994, Proc. SPIE, 2198, 362

Wahhaj, Z., Liu, M. C., Biller, B. A., et al. 2011, ApJ, 729, 139

Webb, R. A., Zuckerman, B., Platais, I., et al. 1999, ApJL, 512, L63

Weinberger, A. J., Anglada-Escudé, G., \& Boss, A. P. 2013, ApJ, 762, 118

West, A. A., Hawley, S. L., Bochanski, J. J., et al. 2008, AJ, 135, 785

White, R. J., Ghez, A. M., Reid, I. N., \& Schultz, G. 1999, ApJ, 520, 811

Wilson, O. C. 1941, ApJ, 93, 29

Wizinowich, P., Acton, D. S., Shelton, C., et al. 2000, PASP, 112, 315

Yamamoto, K., Matsuo, T., Shibai, H., et al. 2013, PASJ, 65, 90

Yee, J. C., \& Jensen, E. L. N. 2010, ApJ, 711, 303

Yelda, S., Lu, J. R., Ghez, A. M., et al. 2010, ApJ, 725, 331

York, D. G., Adelman, J., Anderson, J. E., et al. 2000, AJ, 120, 1579

Zacharias, N., Finch, C. T., Girard, T. M., et al. 2013, AJ, 145, 44

Zacharias, N., Monet, D. G., Levine, S. E., et al. 2005, yCat, 1297, 0

Zickgraf, F.-J., Engels, D., Hagen, H.-J., Reimers, D., \& Voges, W. 2003, A\&A, 406, 535 